\title{
WestVirginiaUniversity
}

THE RESEARCH REPOSITORY @ WVU

Graduate Theses, Dissertations, and Problem Reports

2005

\section{Gallium nitride-based blue laser diodes}

Sridhar Kuchibhatla

West Virginia University

Follow this and additional works at: https://researchrepository.wvu.edu/etd

\section{Recommended Citation}

Kuchibhatla, Sridhar, "Gallium nitride-based blue laser diodes" (2005). Graduate Theses, Dissertations, and Problem Reports. 4164.

https://researchrepository.wvu.edu/etd/4164

This Thesis is protected by copyright and/or related rights. It has been brought to you by the The Research Repository @ WVU with permission from the rights-holder(s). You are free to use this Thesis in any way that is permitted by the copyright and related rights legislation that applies to your use. For other uses you must obtain permission from the rights-holder(s) directly, unless additional rights are indicated by a Creative Commons license in the record and/ or on the work itself. This Thesis has been accepted for inclusion in WVU Graduate Theses, Dissertations, and Problem Reports collection by an authorized administrator of The Research Repository @ WVU. For more information, please contact researchrepository@mail.wvu.edu. 


\title{
GALLIUM NITRIDE BASED BLUE LASER DIODES
}

\author{
Sridhar Kuchibhatla \\ Thesis submitted to the \\ College of Engineering and Mineral Resources \\ at West Virginia University \\ in partial fulfillment of the requirements \\ for the degree of \\ Master of Science \\ in \\ Electrical Engineering
}

Dimitris Korakakis, Ph.D., Chair

Lawrence A Hornak, Ph.D.

Mark A Jerabek, Ph.D.

Lane Department of Computer Science and Electrical Engineering

Morgantown, West Virginia

2005

Keywords: Blue laser diodes, Quantum well, ICP-RIE

Copyright 2005 Sridhar Kuchibhatla 


\section{ABSTRACT \\ GALLIUM NITRIDE BASED BLUE LASER DIODES Sridhar Kuchibhatla}

Group III nitride wide band gap semiconductors have recently attracted considerable attention due to their applications for optical devices operating in the blue and UV wavelength regions. Nitride materials are stable at high temperatures and also chemically stable. Analysis of InGaN based blue laser diodes is required for the research and development of future deep UV laser diodes for bio-sensing applications. The conventional multiple quantum wells suffer from inhomogeneous carrier distribution across quantum wells. This inhomogeneous distribution increases the threshold current density of the laser diode. This project addresses the issue through the design of a novel structure for the active region of InGaN blue laser diodes. As a possible replacement for the AlGaN cladding layers optical properties of a 1D photonic crystal was investigated. Part of the work deals with the etching of $\mathrm{GaN}$ in $\mathrm{SF}_{6}$ plasma using ICP-RIE systems. 


\section{ACKNOWLEDGEMENTS}

I would like to express my sincere thanks to my advisor Dr.Dimitris Korakakis for providing me with an opportunity to work on nitride based laser diodes, for his guidance and support which motivated me to come up with novel designs. I would also like to thank Dr.Lawrence Hornak and Dr.Mark Jerabek for their support and suggestions for the improvement of the design.

I also wish to thank Dr.Kolin Brown for training me on the lab equipment and for helping me to analyze the experimental results.

I would like to acknowledge Smiti, Kalyan, Divya, Lavanya and all my lab colleagues for their support and timely help for the completion of my lab experiments.

Last but not the least I would like to acknowledge Dr.Cooley and Ms.Lucy Freed for their constant support, encouragement and guidance through out my Masters program. 


\section{DEDICATION}

It is with a deep sense of pride and joy that I dedicate this work to my mother Mrs K.Leelavathi, father Mr K.V. Avadhanulu ,to my sisters Sirisha and Usha as without their support I wouldn't have been able to come this far. I would also like to dedicate my thesis to Mr.Kalyan Reddy Kasarla, who has been of great support to me all through my career. 


\section{TABLE OF CONTENTS}

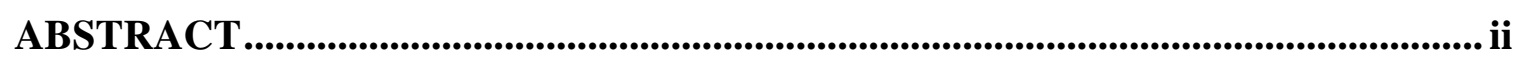

ACKNOWLEDGEMENTS ........................................................................................... iii

DEDICATION................................................................................................................ iv

TABLE OF CONTENTS ......................................................................................

LIST OF FIGURES ......................................................................................... vii

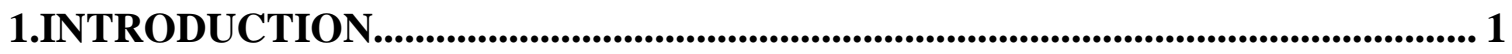

2.LITERATURE REVIEW ................................................................................................ 3

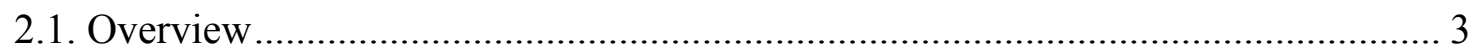

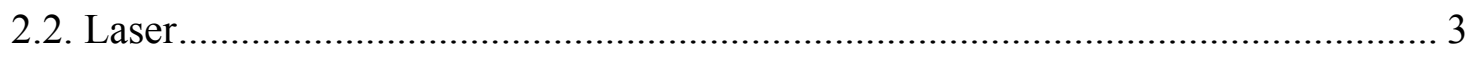

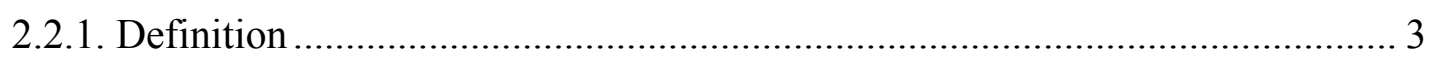

2.2.2. Amplification of stimulated emission in a Fabry -Perot cavity ...................... 5

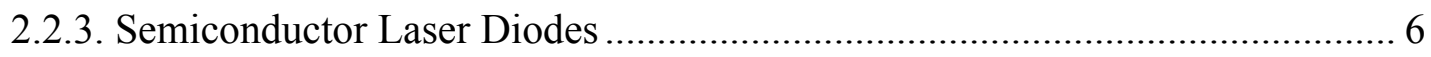

2.2.4. Types of Semiconductor laser Diodes .......................................................... 7

2.2.4.1 P-N junction Laser Diode .................................................................. 7

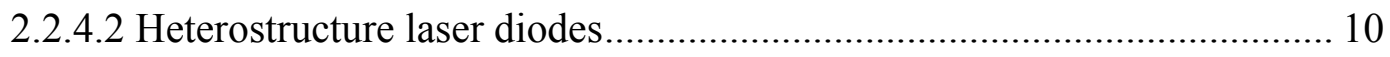

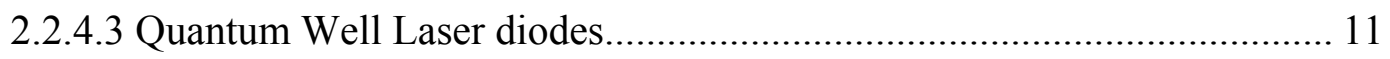

2.2.5. Characteristics of an edge emitting laser diode .............................................. 17

2.2.5.1. Longitudinal Mode.............................................................................. 17

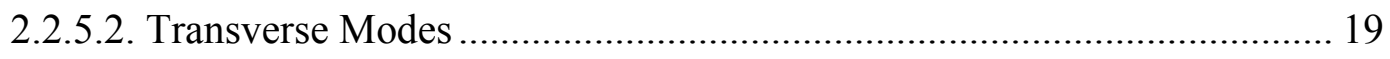

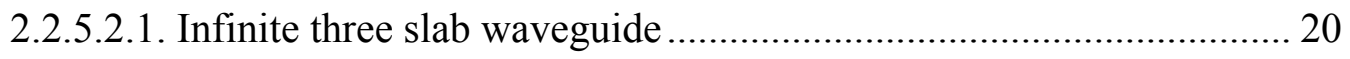

2.2.5.2.2. Single transverse mode condition for both TE and TM modes ........ 22

2.2.5.2.3. Confinement of the electric field to the coreTE $\mathrm{E}_{00}$ mode for our design .

2.2.5.2.4. Gaussian Beam.............................................................................. 23

2.2.6 Output power vs Current characteristics...................................................... 25

2.2.7. Applications of semiconductor laser diodes ................................................. 25

2.2.8. Blue Laser Diodes................................................................................... 26

2.2.8.1 Blue laser diode applications .............................................................. 26 
2.2.8.2. Materials used for blue laser diodes.................................................... 26

2.2.8.2.1. Wide bandgap materials for blue laser diodes ............................. 26

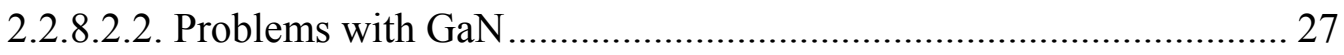

2.2.8.3. Group III-V based InGaN/AlGaN Multi Quantum Well laser diodes ..... 27

2.2.8.3.1. Bandgap properties and Optical properties of InGaN..................... 28

2.2.8.3.2. Issues related to the indium phase segregation - quantum dots ....... 29

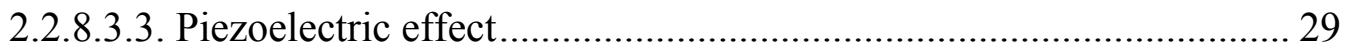

2.2.8.3.4. Variation of bowing parameter with Indium mole fraction .............. 30

2.2.8.3.5. Bandgap and Optical properties of AlGaN .................................. 31

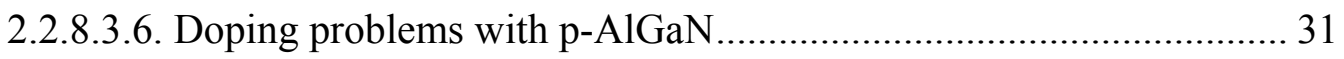

2.2.8.2.7. Structure of InGaN laser diode .................................................. 32

3. PROPOSED STRUCTURE FOR BLUE LASER DIODE .................................. 35

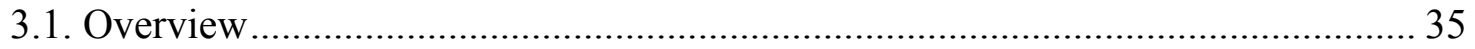

3.2. Design of MQW InGaN blue laser diode .................................................... 35

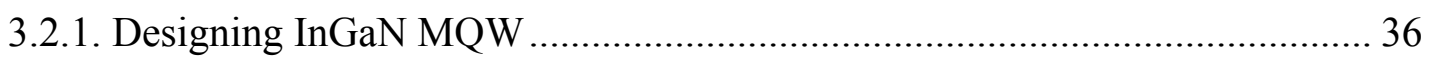

3.2.1.1. Emission of blue wavelength $(440 \mathrm{~nm}-480 \mathrm{~nm})$................................. 36

3.2.1.2. Designing GaN Optical guiding region \& AlGaN Cladding region........ 39

3.2.2. Proposed structures for the active region quantum wells ............................ 41

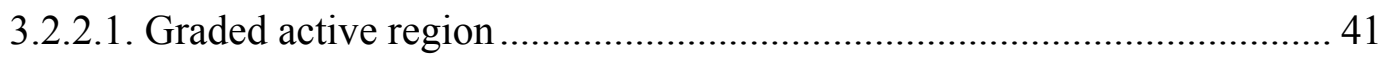

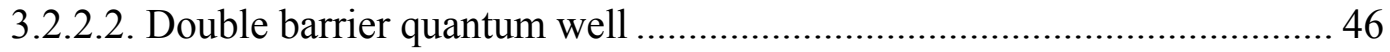

3.2.3. Novel structure for cladding layer 1D Photonic Crystal (PC) ...................... 49

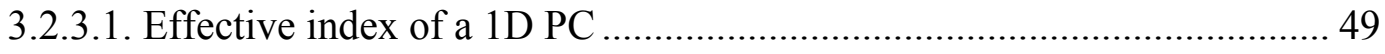

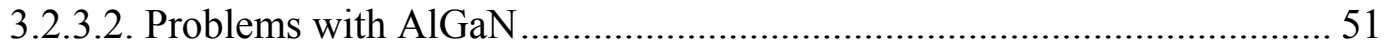

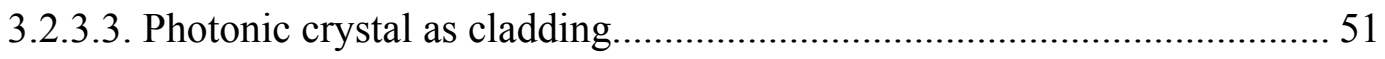

4. ETCHING TECHNIQUES FOR GaN .......................................................... 52

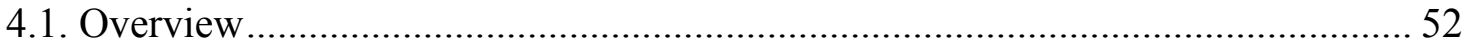

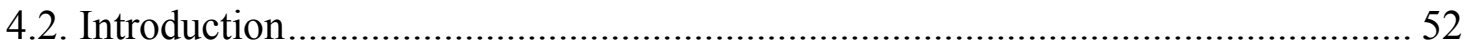

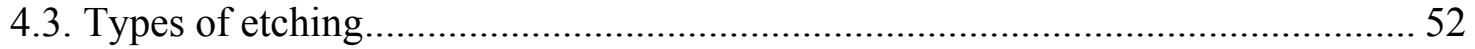

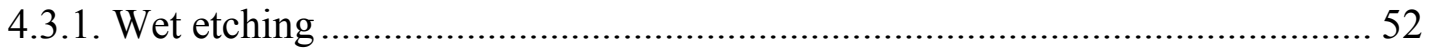




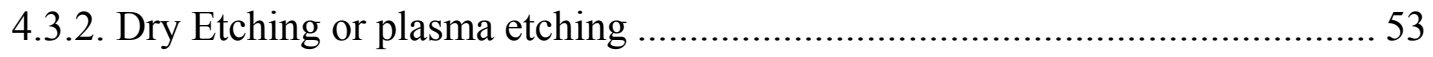

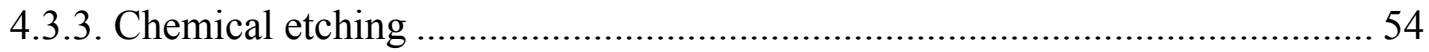

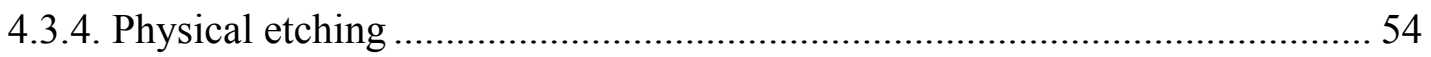

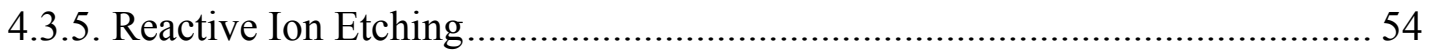

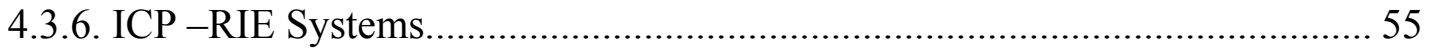

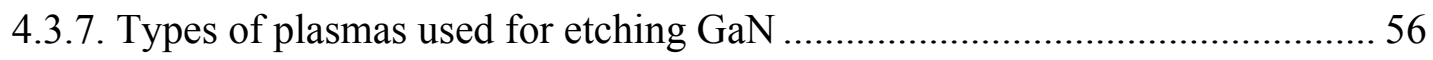

5.EXPERIMENT-ETCH RATE OF GaN ................................................................. 58

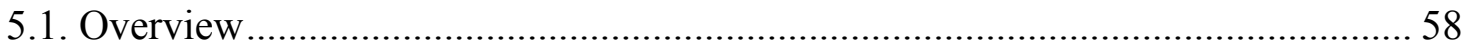

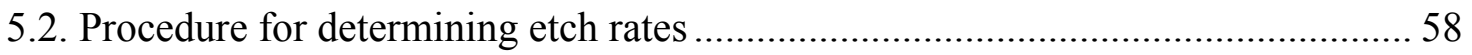

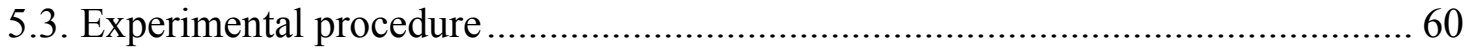

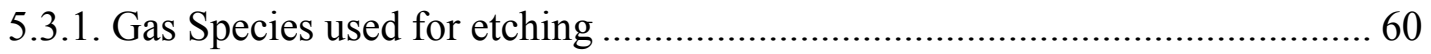

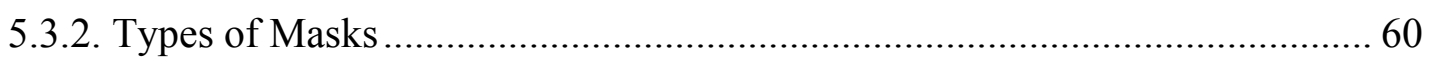

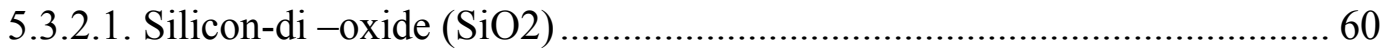

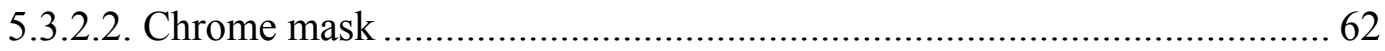

6. CONCLUSIONS AND FUTURE WORK ..........................................................67

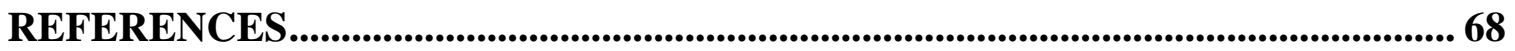

APPENDIX A: SOLUTION FOR INDIUM MOLE FRACTION OF THE GRADED ACTIVE REGION-USING MATLAB PROGRAM ............................................. 74

APPENDIX B - SOLUTION FOR THE LOWEST ENERGY IN THE CONDUCTION BAND OF THE WIDER QUANTUM WELL IN A DOUBLE BARRIER QUANTUM WELL-USING MATLAB PROGRAM ............................ 86

APPENDIX C:SOLUTION TO 1D TIME-INDEPENDENT SCHRODINGER WAVE QUATION USING MATLAB. 94 
APPENDIX D: PROTOCOL FOR DEPOSITING STRIPES OF CHROME ON

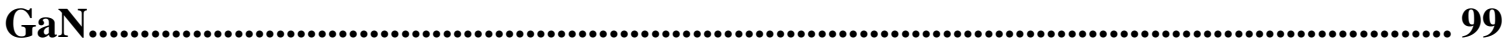




\section{LIST OF FIGURES}

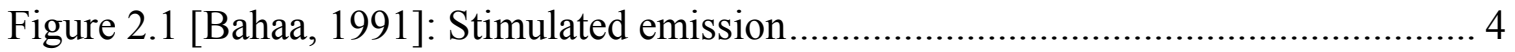

Figure 2.2 [Bahaa, 1991]: Spontaneous Emission .................................................. 5

Figure 2.3 [Bahaa, 1991]: Laser action in the active region ........................................ 6

Figure 2.4 [Bahaa, 1991]: Energy band diagram of a direct bandgap semiconductor........ 6

Figure 2.5 [Bahaa, 1991]: Energy band diagram of an indirect bandgap semiconductor .. 7

Figure 2. 6 [Streetman, 2000]: P-N junction diode under forward bias .......................... 8

Figure 2.7 [Bahaa, 1991]: Fermi level in a semiconductor under population inversion .... 8

Figure 2. 8 [Streetman, 2000]: Fermi levels in a p-n junction diode under forward bias... 9

Figure 2. 9 [Coldren, 1995]: Energy levels in double heterostructure material .............. 10

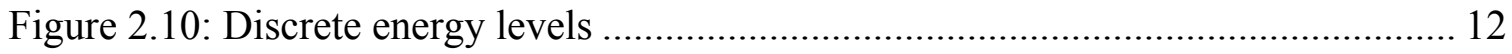

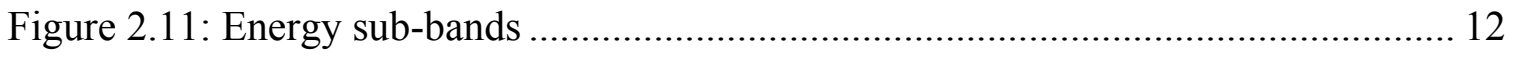

Figure 2.12: Energy levels (tan solutions, lowest energy level in tan solution) in an

InGaN quantum well with GaN barriers of width $5 \mathrm{~nm}$ Indium mole concentration of .22 .

Figure 2.13: TE0 mode profile for a single $\mathrm{GaN}$ quantum well active region with InGaN barriers of width $50 \mathrm{~A}^{\circ}$ and $400 \mathrm{~nm}$ emission wavelength. Simulated using online 1D mode solver

Figure 2.14: TE0 mode profile for a single GaN DHLD active region with InGaN barriers of width $100 \mathrm{~nm}$ and 400nm emission wavelength. Simulated using online 1D mode solver

Figure 2.15 [Coldren, 1995]: Energy levels in an SCH structure

Figure 2.16: Emission spectrum of a laser diode with quantum well and bulk as active regions

Figure 2.17 [Bahaa, 1991]: Longitudinal modes in a fabry-perot cavity........................ 18

Figure 2.18 [Streetman, 2000]: Wavelengths in a fabry perot cavity ............................ 19

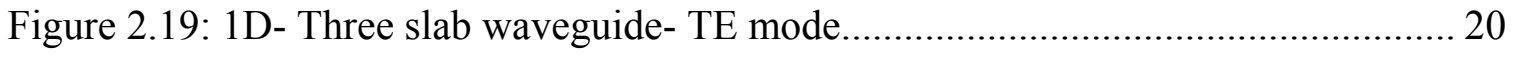


Figure 2.20: TE and TM modes in a three slab waveguide, Intensities of TE and TM profiles for $\mathrm{n} 1=2.5067, \mathrm{n} 2=2.47, \lambda=420 \mathrm{~nm}$. Simulated using online $1 \mathrm{D}$ mode solver

Figure 2.21: Percentage of the optical field confined to the core for varying wavelengths, core thickness and refractive index of cladding $\mathrm{a}=0.5 \mu \mathrm{m}_{1}=2.5067 \mathrm{n}_{2}=2.4760 \lambda$ $=410 \mathrm{~nm}$. Simulated using online 1D mode solver.

Figure 2.22 [Bahaa, 1991]: Gaussian field distribution outside the laser cavity with increasing radial distance 24

Figure 2.23 [Kasap, 2001]: Typical structure of an edge emitting laser diode. 24

Figure 2.24 [Kasap, 2001]: Output power vs injection current of an edge emitting laser diode. 25

Figure 2.25: Energy gap of $\mathrm{InGaN}$ as function of indium mole fraction......................... 28

Figure 2.26 [Chichibu, 1998]: Piezoelectric field in an InGaN quantum well ..... 29

Figure 2.27 [Ferhat, 2001] :Variation of InGaN bandgap bowing as a function of Indium mole fraction ,Bandgap bowing is indicated by dark circles 30

Figure 2.28 [Obata, 2004]: Variation of hole concentration in AlGaN as a function of $\mathrm{Al}$ mole fraction 32

Figure 2.29 [Nakamura, 1996]: Structure of blue laser diode fabricated by Shuji

Nakamura 33

Figure 2.30 [Czyszanowski1, 2001]: Refractive index profiles of advanced nitride laser diodes with electric field intensity in the optical guiding region ........................... 34

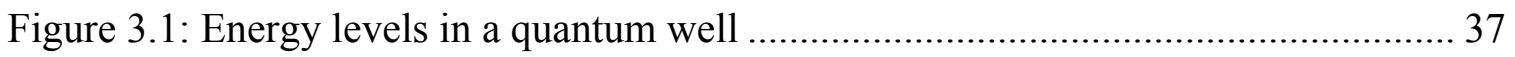

Figure 3.2 Appendix C: Emission wavelength for InN bandgap of $1.9 \mathrm{ev} . . . \ldots \ldots \ldots \ldots \ldots \ldots . . . . . . . . . . .38$

Figure 3.3 Appendix C: Emission wavelength for InN bandgap of $1.9 \mathrm{ev}$...................... 38

Figure 3.4: Theoretical vs experimental data collected form journals for InGaN laser diodes fabricated on MOCVD and operated at RT 39

Figure 3.5: Electric field intensity in the optical guiding region and cladding region ..... 40

Simulated using online 1D mode solver 40

Thickness of Optical guiding region is: $0.22 \mu \mathrm{m}$ 40

Figure 3.6 [Domen, 1998]: Stimulated emission and absorption in a multiple InGaN quantum well. 
Figure 3.7: Effective mass of electrons and light holes as a function of indium mole fraction 43

Figure 3.8: Effective mass of heavy electrons as a function of indium mole fraction ..... 44

Figure 3.9 Appendix A: Variation of well thickness and indium mole fraction in the active region for an emission wavelength of $450 \mathrm{~nm}$

Figure 3.10: Alignment of discrete energy levels in the structure Fig 3.7 conduction band for InN bandgap of 1.9e.v. 45

Figure 3.11: Alignment of discrete energy levels in the conduction band for $\mathrm{InN}$ bandgap of 1.9e.v and different combination of quantum well thickness. 45

Figure 3.12 Appendix B: Simple double barrier for emission wavelength of $450 \mathrm{~nm} . . . . .47$ and for $\mathrm{InN}$ bandgap of 1.9e.v. 47

Figure 3.13 Appendix B: Lowest energy state separation from the edge of barrier2 for emission wavelength of $450 \mathrm{~nm}$ 48

Figure 3.14: Variation of refractive index of a 1D photonic crystal in direction of propagation of light. 50

Figure 3.15: Effective index of 1D photonic crystal $\mathrm{Al}_{0.32} \mathrm{Ga}_{0.68} \mathrm{~N} /$ air for different incidence angles, when incident from GaN 50

Figure 4.1 [Stocker, 1998]: Undercut in GaN resulting from wet etching ....................... 53

Figure 4.2 Oxford Plasma Systems: RIE System ………………………………....... 55

Figure 4.3 Oxford Plasma Systems: ICP-RIE............................................................... 56

Figure 5.1: Unetched Sample ............................................................................... 58

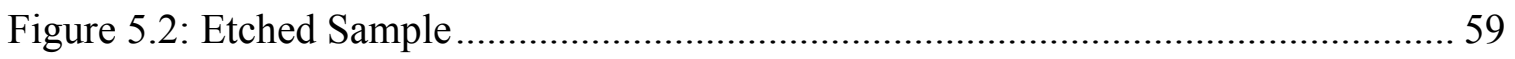

Figure 5.3: Sample after mask removal ................................................................ 59

Figure 5.4: Etch rates of Silicon-dioxide and Photoresist vs. Chamber Pressure............. 61

Figure 5.5 Etch rates of Silicon-dioxide and Photoresist vs. Flow rate of $\mathrm{SF}_{6} \ldots \ldots \ldots \ldots \ldots . . . .61$

Figure 5.6: Etch rate of GaN vs Chamber pressure ....................................................... 62

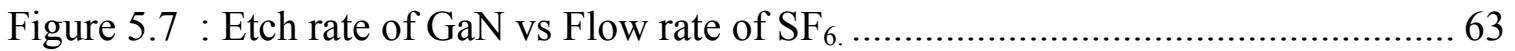

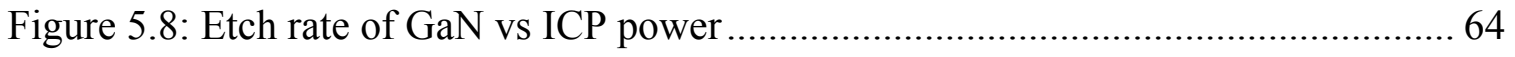

Figure 5.9: $\mathrm{SEM}$ image of $\mathrm{GaN}$ at $\mathrm{ICP}=170 \mathrm{~W}, \mathrm{RF} 150 \mathrm{~W}, \mathrm{FR}=30 \mathrm{sccm}, \mathrm{P}=17 \mathrm{mT}$....... 65

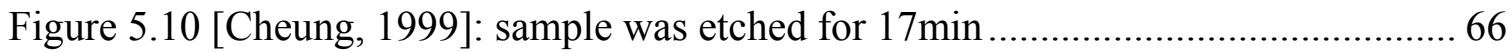




\section{INTRODUCTION}

Semiconductor laser diodes (SLDs) due to their small size, low power dissipation and high quantum efficiency than solid state lasers have tremendous applications in the field of optical data storage, printing, optical communications, spectroscopy etc.

As secondary data storage devices optical discs are preferred over magnetic discs due to unique features such as small size, high data storage capacity, high immunity to external EM interference and therefore a longer life time. Information on optical disks is recorded in the form tiny grooves and planes by SLDs. The capacity of the disc can be improved by increasing aerial density of these grooves. The area of the groove is proportional to the incident light wavelength. The spot $d$ diameter is approximately given by

$$
\mathrm{d}=\lambda / \mathrm{N} . \mathrm{A}
$$

N.A - Numerical aperture of the lens.

Thus the density and at the same time resolution can be improved by a reduction in emission wavelength of the laser diode. Currently infra-red laser diodes which are at affordable prices are being widely used. Also high resolution laser color printers and low cost full color projection systems could be designed by moving towards shorter wavelength laser diodes.

It was therefore necessary to produce lasers which emit shorter wavelength. Research oriented towards materials emitting shorter wavelength has to led to $\mathrm{ZnSe}$ and $\mathrm{GaN}$. These materials have a wide band gap and sufficient to emit light in the ultraviolet region

and by forming alloys with other materials the band gap could be varied. Inspite of having a high defect density GaN based materials have been found to exhibit a higher quantum efficiency and longer life time when compared to laser diodes based on $\mathrm{ZnSe}$ [Ueta, 2004]. Various reasons like formation of quantum dots, periodic variations in the quantum well thickness of the active region have been attributed for this high quantum efficiency [Narukawa, 1996], [Narayan, 2002]. GaN can be alloyed with other material 
systems like $\mathrm{InN}$ and AlN to obtain a range of emission wavelengths. The emission wavelength can be changed by varying the Indium and Aluminium concentrations.

Study of characteristics of InGaN blue laser diode is important in understanding the behavior of deep UV laser diodes fabricated using AlInGaN [Akita, 2004]. Deep UV lasers find applications in biosensing such as detecting chemical agents.

Efforts are being directed towards improving the performance of $\mathrm{GaN}$ based laser diodes by improving the crystal quality of $\mathrm{GaN}$, using different substrates, improving the p-GaN contacts, reducing the overflow current by modifying the waveguide structure [Kuramata,1999],[Song ,2004],[ Bour,2000].

ICP-RIE technique has been used for anisotropic etching GaN laser diodes for vertical and smooth side walls due to its selectivity and low damage to the device. 


\section{LITERATURE REVIEW}

\subsection{Overview:}

This chapter discusses the operation of a semiconductor laser diode, characteristics of a fabry-perot laser diode. The necessity of a separate confinement heterostructure $(\mathrm{SCH})$ for a quantum well laser diode and the evolution of blue laser diodes are discussed.

\subsection{Laser:}

\subsubsection{Definition:}

Laser stands for Light amplification by stimulated emission of radiation. A system having exited electrons is not in an equilibrium state or it is in an unstable state. To attain stability the electrons relax to the ground state by losing energy in the form of a photon or phonon. In the case of a photon if:

$\mathrm{E}_{\mathrm{g}}$ - Energy of the electron in ground state.

$\mathrm{E}_{\mathrm{e}}$ - Energy of the electron in excited state.

h - Plank's constant.

c - Velocity of light in vacuum.

$\lambda$ - Wavelength of the emitted EM wave.

$$
\text { Energy of the emitted photon } \Delta \mathrm{E}=\mathrm{E}_{\mathrm{g}}-\mathrm{E}_{\mathrm{e}}=\frac{h c}{\lambda}
$$

The emission mechanism for an unstable system can be either stimulated or spontaneous emission.

Stimulated emission: In this mechanism photons induce electrons to loose energy by forcing them to emit photons. The emitted photons have the same frequency, phase and polarization. This occurs when the density of electrons in excited state is more than the density of electrons in ground state. This is called population inversion, an unstable state. 


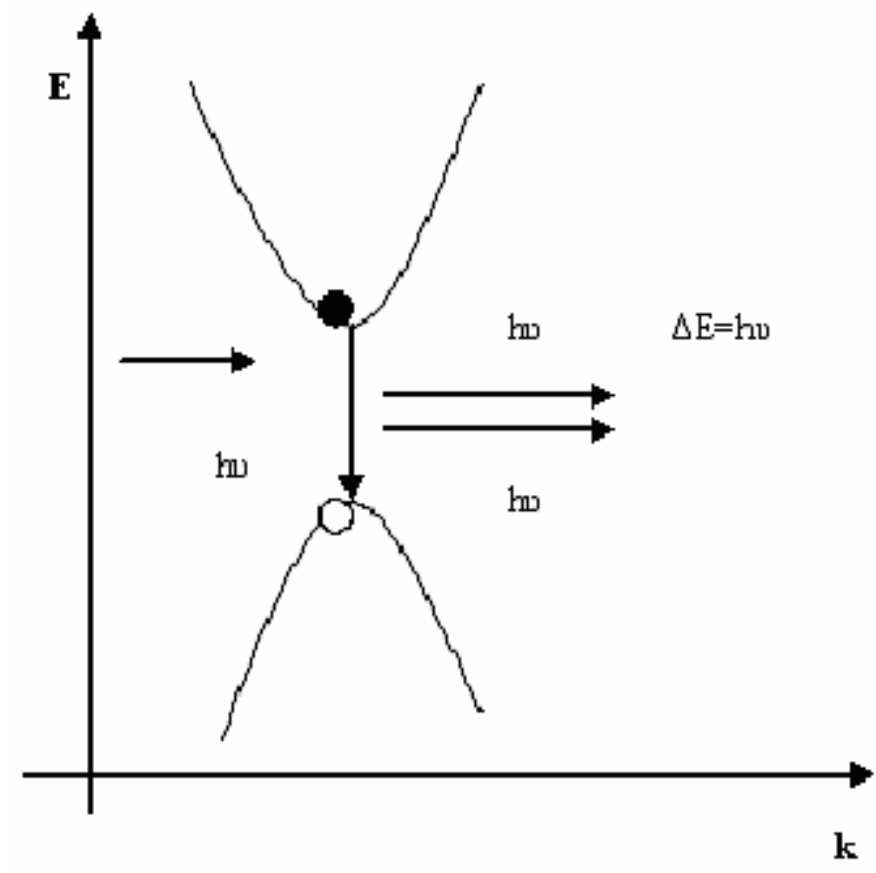

Figure 2.1 [Bahaa, 1991]: Stimulated emission

Spontaneous emission: In this mechanism the photons are emitted randomly without the interference from other photons. The emitted photons have random directions and polarizations in other words they are incoherent. Population inversion is not needed in this case. The electrons emit light without coupling from external photons based on their relaxation time. 


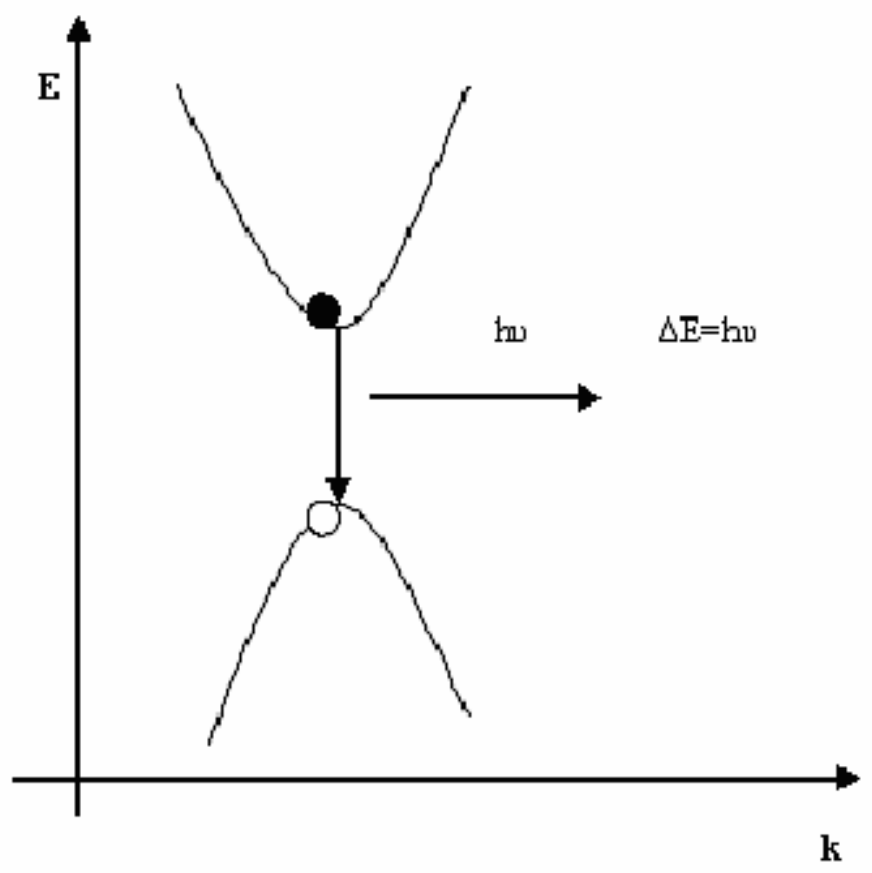

Figure 2.2 [Bahaa, 1991]: Spontaneous Emission

\subsubsection{Amplification of stimulated emission in a Fabry -Perot cavity:}

To amplify the stimulated emission, in order to get a coherent beam, an optical feedback network that consists of a highly reflecting mirror and a partially reflecting mirror at either end of the active region is provided. The emitted light is amplified several times before leaving the active region. Lasing occurs when the net gain for a roundtrip in the cavity is more than the losses. Major losses include absorption of optical absorption along the cavity length and losses through the partially reflecting mirror. The system acts as an active region and is the core. It also acts as the guiding layer for the light. 


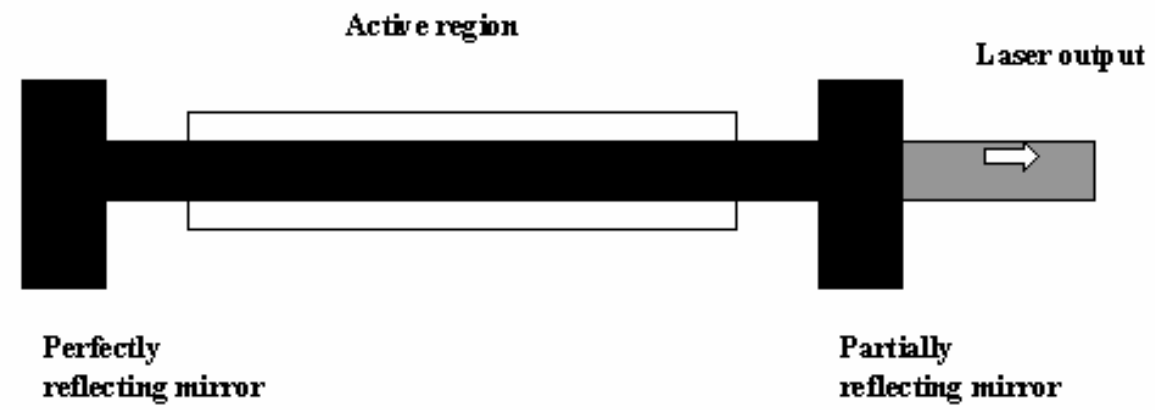

Figure 2.3 [Bahaa, 1991]: Laser action in the active region

\subsubsection{Semiconductor Laser Diodes:}

Emission of light in the core for a semiconductor laser diode is through the process of recombination of electrons in the conduction band with the holes in the valence band. Semiconductors having a direct band gap are capable of emitting photons.

Direct band gap: The conduction band minimum and the valence band maximum have the same wave vector that is at $\mathrm{k}=0$ Fig 2.4. Since by definition the highest energy of the valence band is set at $\mathrm{k}=0$.

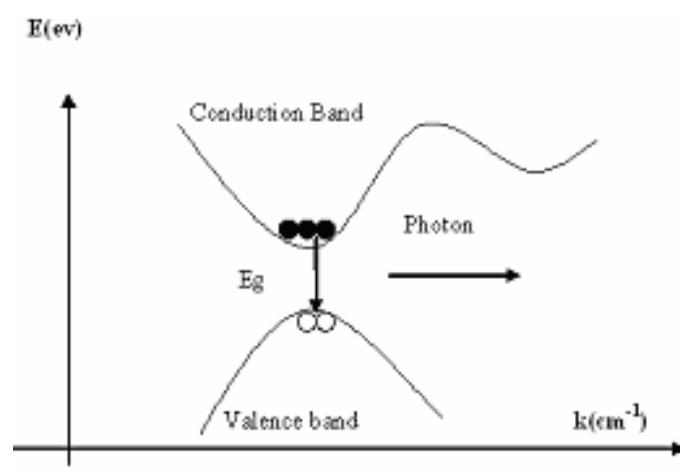

Figure 2.4 [Bahaa, 1991]: Energy band diagram of a direct bandgap semiconductor

Indirect band gap: The conduction band minimum and valence band maximum are at a different wave vector Fig 2.5. 


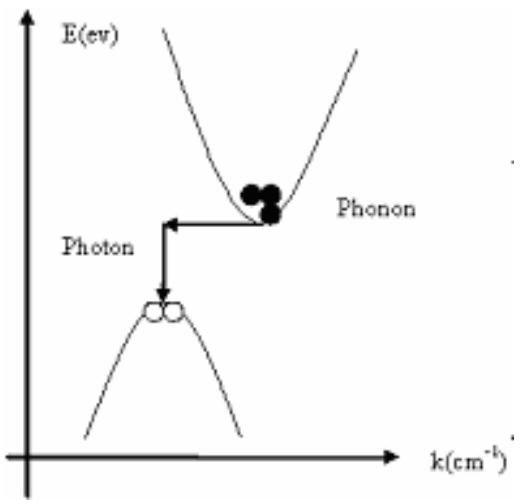

Figure 2.5 [Bahaa, 1991]: Energy band diagram of an indirect bandgap semiconductor

\section{Conservation of momentum and energy of a photon:}

Efficient emission of photons is possible only in direct band gap semiconductors.

An electron changing state must have both energy and momentum conserved. In case of direct band gap semiconductors there is little change in momentum between the two states so energy is given up in the form of photons. In the case of indirect band gap semiconductors the electron must loose energy in and transfer momentum. This is achieved through lattice vibrations in the form of phonons. The probability of electrons losing momentum and still emitting photons at the desired wavelength is very low [Bahaa,1991].

\subsubsection{Types of Semiconductor laser Diodes:}

\subsubsection{P-N junction Laser Diode:}

A simple laser diode can be constructed using a direct band gap semiconductor material. The diode consists of heavily doped p-n regions and highly reflecting facets. The p-n junction acts as the active region. When the diode is forward biased electrons and holes recombine at the junction producing photons Fig 2.6. When a sufficiently large forward bias voltage is applied across the diode it leads to accumulation of a large number of carriers around the junction leading to conditions for population inversion at the junction and hence stimulated emission. 


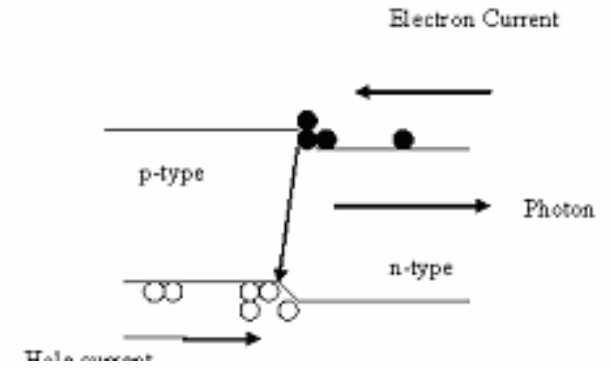

Figure 2. 6 [Streetman, 2000]: P-N junction diode under forward bias

\section{Conditions for stimulated emission in a semiconductor:}

Unlike a solid state laser viz Nd-Yag, where there are two, three or four level systems, a semiconductor has a continuum of bands and the condition for population inversion is different in a $\mathrm{p}-\mathrm{n}$ junction laser diode. The condition for stimulated emission for a semiconductor with energy gap Eg is:

$$
\mathbf{E}_{\mathbf{F}}^{\mathrm{e}}-\mathbf{E}_{\mathbf{F}}^{\mathrm{h}} \geq \mathbf{E}_{\mathbf{g}} \geq \mathbf{h} \boldsymbol{V}
$$

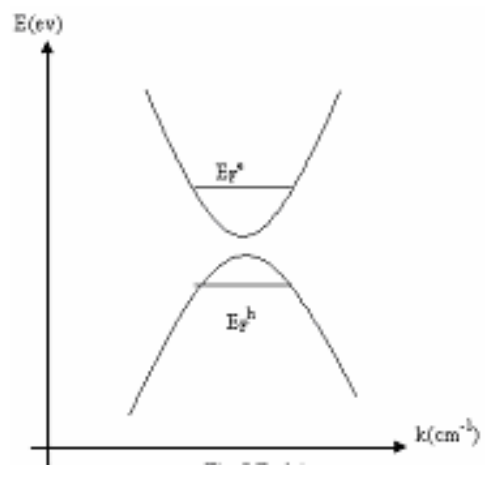

Figure 2.7 [Bahaa, 1991]: Fermi level in a semiconductor under population inversion

$\mathbf{E}_{\mathbf{F}}{ }^{\mathbf{e}}$ - Fermi level in n type.

$\mathbf{E}_{\mathbf{F}}{ }^{\mathbf{h}}-$ Fermi level in p-type.

Ec-Conduction band edge.

$\mathbf{E v}$ - valence band edge. 


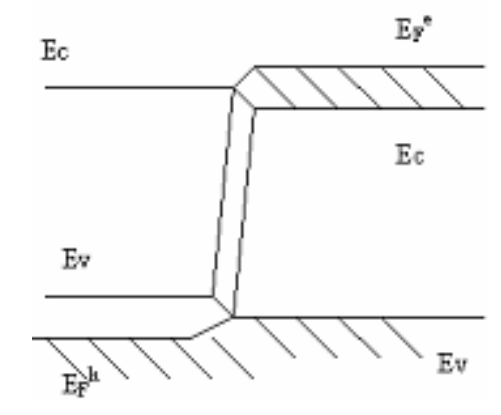

Figure 2. 8 [Streetman, 2000]: Fermi levels in a p-n junction diode under forward bias

Lasing occurs when the net gain along the plane of the junction equals the losses. The minimum current at which stimulated emission overcomes the losses leading to a lasing action is called the threshold current density.

\section{Amplification along the stripe of an edge emitting laser diode:}

Most edge emitting laser diodes have stripe geometry, where the metallic contacts extend along the length of the resonant cavity. The stimulated emission is amplified only along the direction of the stripe. In other directions the there is no population inversion for supporting stimulated emission. More photons would stimulate the electrons to emit light along the stripe; therefore light is amplified only along the stripe. In stimulated emission the photons force the excited electrons to emit photons having the same polarization and this leads to maximum gain in a particular direction i.e. along the length of the stripe geometry. In spontaneous emission photons are emitted in random directions. The intensity of emitted light is very low. As fewer photons are generated in the cavity due to the absence of stimulated emission and also due to the fact that emitted photons have random directions, so the majority of the photons do not leave the cavity at the same time.

\section{Disadvantages of a p-n junction laser diode:}

A simple $\mathrm{p}-\mathrm{n}$ junction laser diode has the disadvantage of maximum optical power spread out of the active region due to less difference in refractive index between active region and the material surrounding it thereby leading to improper optical confinement. 


\subsubsection{Heterostructure laser diodes:}

It is difficult to achieve population inversion at low currents for a simple p-n junction diode as the carriers tend to diffuse away from the junction also the optical field is not confined to the active region. Therefore the losses are high. So a large current is required to achieve population inversion and hence lasing action. These problems can be overcome by using a heterostructure.

A heterostructure junction is formed by joining a lower band gap semiconductor with a higher band gap semiconductor. A double heterostructure laser diode (DHLD) is formed by sandwiching a lower bandgap material in between two higher bandgap materials, called the cladding layers. In most cases the band gap of the materials on either side of are equal. This structure acts as a potential well Fig 2.9.

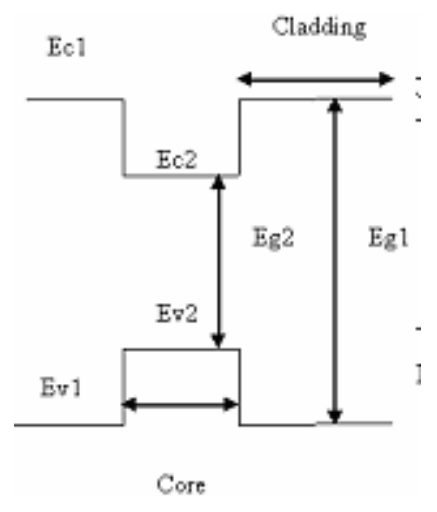

Figure 2. 9 [Coldren, 1995]: Energy levels in double heterostructure material

Under forward bias electrons from n-region and holes from the p- region are injected into the lower bandgap region which acts as the active region or core. The injected carriers are trapped in the potential well as they do not have sufficient energy to cross the energy barrier. When sufficient numbers of carriers are pumped into the active region population inversion occurs in the lower bandgap material leading to stimulated emission.

\section{Confinement of light in a DHLD}

A DHSLD is far more efficient than a simple p-n junction laser diode since it traps the carriers in a well like structure and prevents them from diffusing out, thereby spatially confining them thus increasing the probability of their recombination[Bahaa, 1991]. The 
layer with the lower band gap has a higher refractive index when compared to the regions having a higher band gap. Also due to the difference in refractive index of the two regions this structure doubles up as a waveguide. Light is efficiently confined in the core region due to total internal reflection. This minimizes the spreading of the optical field out of the core and also minimizes the losses. These factors contribute to achieving a lower threshold current density at lower current densities when compared to a simple p-n junction laser diode.

\subsubsection{Quantum Well Laser diodes:}

As the thickness of the quantum well decreases the volume of the active region to be pumped for achieving population inversion decreases, in other words the number of states needed to achieve population inversion decreases; thereby population inversion is achieved at lower current densities that may lead to lower threshold current densities.

\section{Quantum Well:}

In a bulk material there is a continuum of energy states. As the thickness of the material is reduced the number of atoms per unit volume decreases. The difference between the energy levels is too high to be considered as continuum of energy states. In such situations we can expect subands of energy levels which are nothing but discrete energy levels. If the material of such thickness is sandwiched between materials having a higher energy gap we end up with a potential well which has discrete energy levels in it which is called a quantum well Fig 2.10, Fig 2.11. 


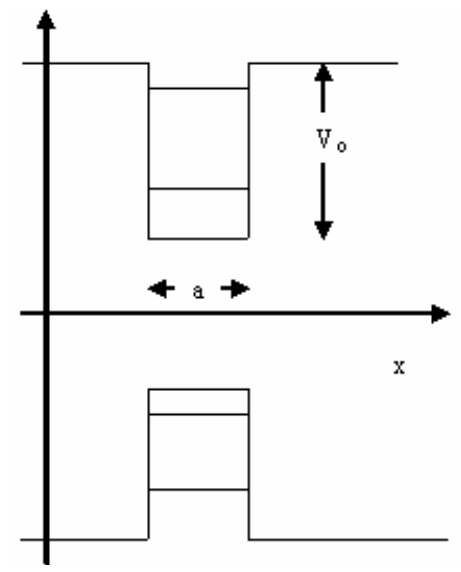

Figure 2.10: Discrete energy levels

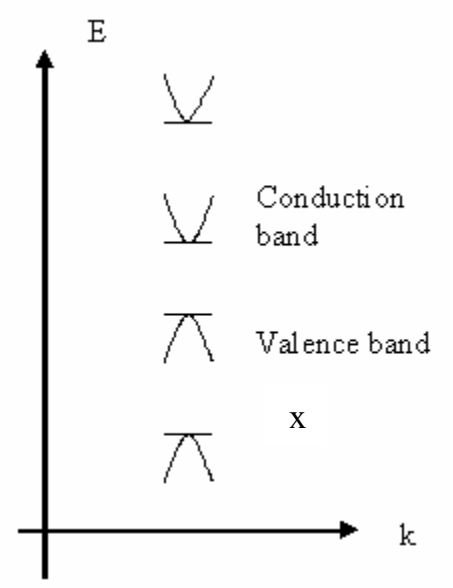

Figure 2.11: Energy sub-bands

The quantum well has additional features viz:

1. Reduced density of states when compared to bulk structures.

2. Discrete energy levels.

3. Constant density of states in individual sub bands.

\section{Calculating Energy levels:}

The energy levels in the conduction band and valence are determined by solving the time 1D time independent Schrödinger equation for a finite potential quantum well subjected to boundary conditions. 
Schrödinger equation is a mathematical model which describes the behavior of a system as a function of spatial and time co-ordinates in terms of a wave function $\Psi$. Solving the Schrödinger equation in time independent form gives the possible wave functions that can exist within the region. Different $\Psi$ functions give the possible energy levels in the region.

$$
-\frac{\hbar^{2}}{2 m} \frac{\partial^{2} \psi(x, t)}{\partial x^{2}}+U(x) \psi(x, t)=i \hbar \frac{\partial \psi(x, t)}{\partial}
$$

This equation can be separated into Time independent and time dependent.

The Time Independent Schrödinger Equation is given by:

$$
-\frac{\hbar^{2}}{2 m} \frac{\partial^{2} \psi(x)}{\partial^{2} x}+U(x) \psi(x)=E \psi(x)
$$

$E$ is the energy of the system.

Carriers at the edge of energy bands follow a parabolic E-k relationship. They behave as free carriers as observed for uniform potentials. The carriers encounter no potentials inside the well. The carriers encounter potential barriers, $V_{o}$, at the extremes of the low bandgap semiconductor.

\section{Boundary Conditions:}

For $|\mathrm{x}|<\mathrm{a} / 2$ the potential $\mathrm{V}(\mathrm{x})=0$.

$$
\begin{aligned}
& \mathrm{U}(\mathrm{x})=0 . \\
& \frac{-\hbar^{2}}{2 m} \frac{d^{2} \psi(x)}{d x^{2}}+0 \psi(x)=E \psi(x) \\
& \frac{d^{2} \psi(x)}{d x^{2}}=-k^{2} \psi(x) \\
& k^{2}=\frac{2 m E}{\hbar^{2}}
\end{aligned}
$$

General solution is of the form

$\Psi=\mathrm{A} \operatorname{Sinkx}+\mathrm{B} \operatorname{Coskx}$

For $|x|>=a / 2$ the potential $V(x)=V_{o}$.

Since $\mathrm{V}(\mathrm{x})$ is symmetric then the solution can be either odd or even. 
Solution in the well can be ASinkx or BCoskx.

In the barrier region

$$
\frac{d^{2} \psi}{d x^{2}}-\frac{2 m}{\hbar^{2}}\left(V_{o}-E\right) \psi=0 \quad \gamma=\frac{\sqrt{2 m\left(V_{o}-E\right)}}{\hbar}
$$

General solution is of the form

$\psi(x)=E e^{-\gamma x}$

\section{Even Solution:}

The wavefunction and its derivatives must be continuous

The boundary conditions give us:

$C_{1}=C_{2} \cos k_{1} \frac{w}{2}$

$\frac{k_{2}}{m_{3}} C_{1}=\frac{k_{1}}{m_{w}} C_{2} \sin k_{1} \frac{w}{2}$

Solving 2.6 and 2.7 we get the quantization condition:

$k_{2}=\frac{m_{3} k_{1}}{k_{w}} \tan k_{1} \frac{w}{2}$

\section{Odd Solution:}

Boundary conditions give us:

$$
\begin{aligned}
& C_{1}=C_{2} \sin k_{1} \frac{w}{2} \\
& \frac{-k_{2} C_{1}}{m_{3}}=\frac{k_{1}}{m_{w}} C_{2} \cos k_{1} \frac{w}{2}
\end{aligned}
$$

The quantization condition:

$$
k_{2}=-\frac{m_{3} k_{1}}{m_{w}} \cot k_{1} \frac{w}{2}
$$


Now a plot with $\mathrm{E}$ as a variable must satisfy the quantization condition. The value of $\mathrm{E}$ at which the equations turn zero gives us the energy levels of the system.
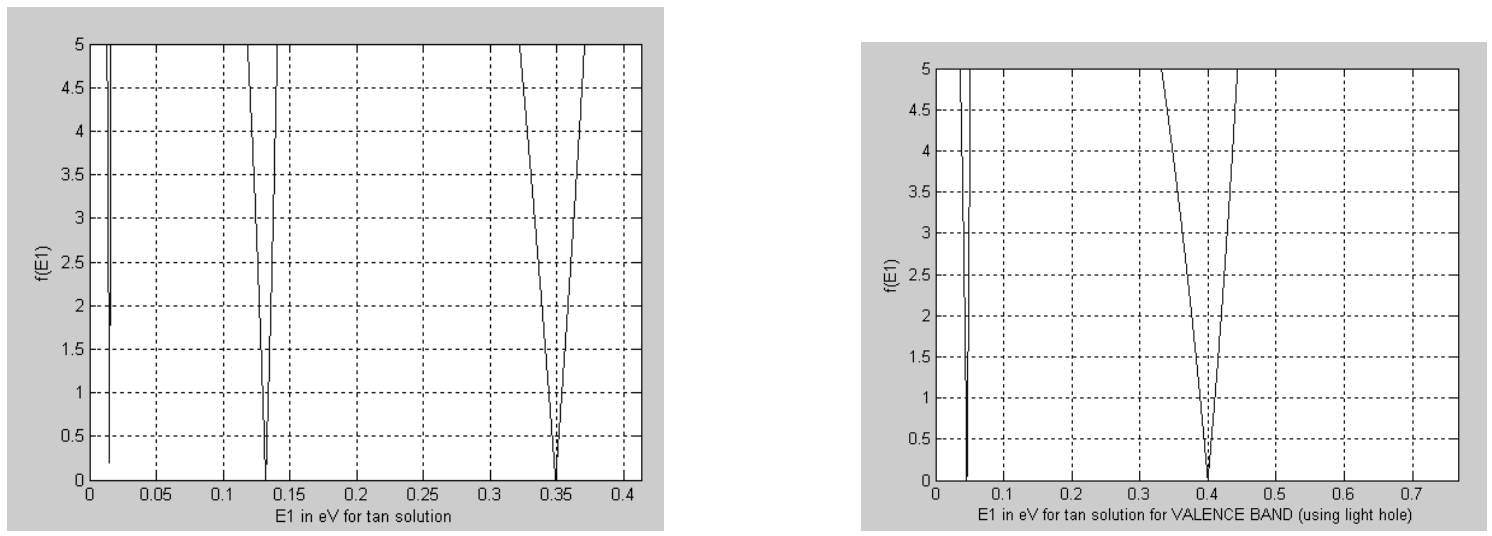

Figure 2.12: Energy levels (tan solutions, lowest energy level in tan solution) in an InGaN quantum well with GaN barriers of width $5 \mathrm{~nm}$ Indium mole concentration of .22

From the plots we see that the energy levels are discrete and these materials behave as quantum wells.

\section{Threshold carrier density of DHLD and quantum well structure:}

The carrier recombination in bulk DHLD is spread over a wide range of wavelengths but in a quantum well based laser diode the carrier recombination is spread over a relatively narrow range of wavelengths. In a quantum well the gain required to achieve lasing therefore requires fewer carriers than bulk.

\section{Effective bandgap of quantum well:}

The effective band gap of the active region also increases as there are no states below the lowest energy level both in the conduction and valence band.

Band gap of bulk for same material $=\mathrm{E}_{\mathrm{g}}$

In a quantum well of the same material, if e1 and e 2 are the lowest and highest energy levels in the conduction and valence band respectively then

The effective band gap of the quantum well $=\mathrm{e} 1+\mathrm{e} 2+\mathrm{E}_{\mathrm{g}}$ 


\section{Confinement of optical field with decreasing thickness of the active region:}

The quantum wells because of their extremely small thickness when compared to the wavelength of the emitted light cannot confine the light properly Fig 2.13. In fact the active region thickness cannot be decreased beyond a certain point as this would lead to inefficient optical confinement increasing the losses and also the threshold current density.

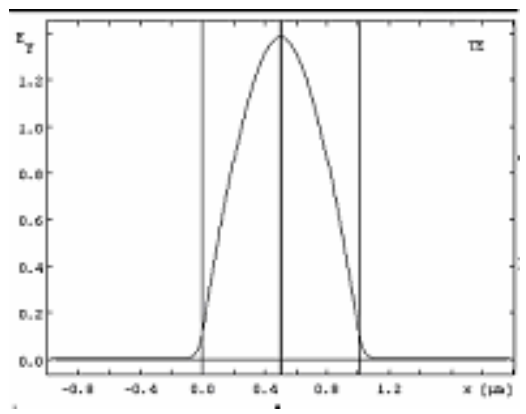

Figure 2.13: TE0 mode profile for a single GaN quantum well active region with InGaN barriers of width $50 \mathrm{~A}^{\circ}$ and $400 \mathrm{~nm}$ emission wavelength. Simulated using online 1D mode solver

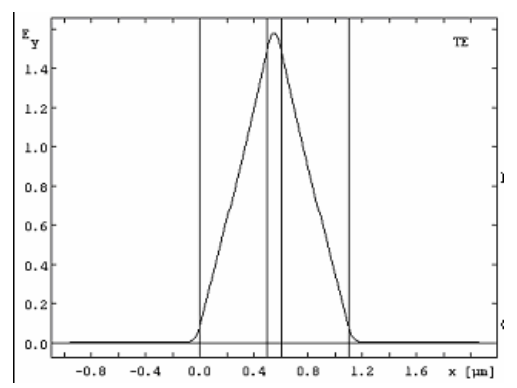

Figure 2.14: TE0 mode profile for a single GaN DHLD active region with InGaN barriers of width $100 \mathrm{~nm}$ and $400 \mathrm{~nm}$ emission wavelength. Simulated using online 1D mode solver

A separate confinement heterostructure ( $\mathrm{SCH})$ Fig 2.14 confines the carriers and the light in separate regions. The carrier confining region was the quantum well and the optical guiding region was called the gain guiding region. 


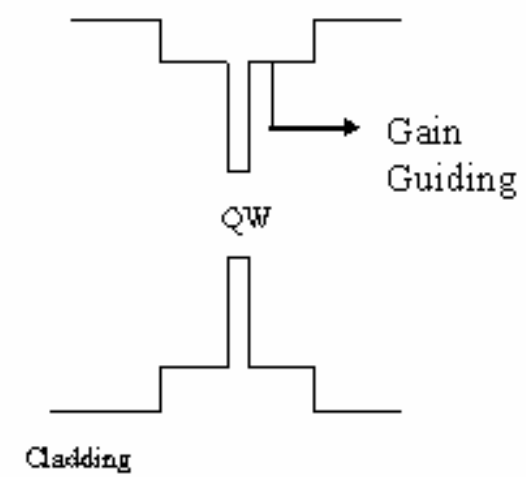

Figure 2.15 [Coldren, 1995]: Energy levels in an SCH structure

\subsubsection{Characteristics of an edge emitting laser diode:}

\section{Modes in the laser diode:}

Depending on the wavelength and the electric field distribution there are two modes existing in a laser diode cavity a longitudinal mode and a transverse mode.

\subsubsection{Longitudinal Mode:}

This mode determines the possible wavelengths which the laser diode cavity supports. An emitted wave having a wavelength $\lambda$ is supported by a cavity of length ' 1 ' only when $1=\mathrm{q}(\lambda / 2 \mathrm{n})$. This condition gives rise to standing waves

where

$\mathrm{q}$ is an integer having values from $\mathrm{q}=1,2,3 \ldots \ldots \ldots$

$\mathrm{n}$ refractive index of the medium

$\lambda$ Free space wavelength.

A relative comparison of the emission spectrum envelope of a bulk and quantum well laser diode would look as follows Fig 2.14: 


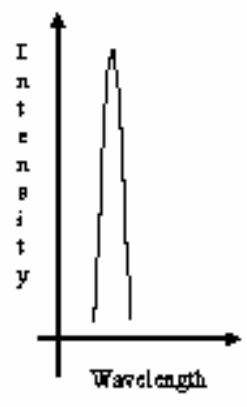

QW

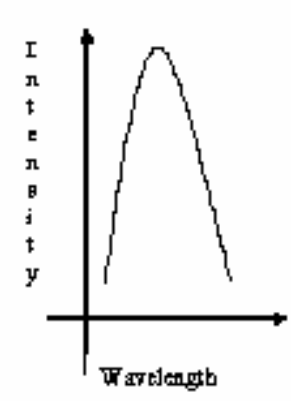

Bulk

Figure 2.16: Emission spectrum of a laser diode with quantum well and bulk as active regions

Waves which do not follow the above conditions interfere destructively. Lasing is not supported for these wavelengths because of no gain during roundtrip.

Given the refractive index and the length of the laser cavity the number of modes ' $\mathrm{m}$ ' and the spectral separation of these modes can be determined.

$$
d \lambda=\left[\frac{\lambda^{2}}{2 n l}\right]
$$

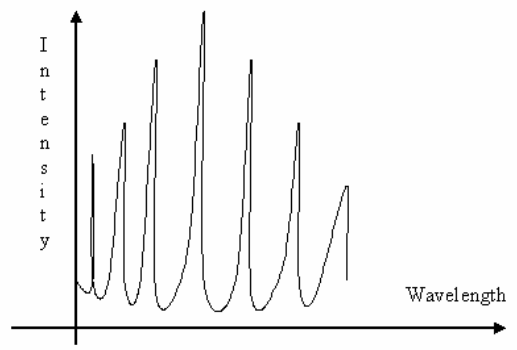

Figure 2.17 [Bahaa, 1991]: Longitudinal modes in a fabry-perot cavity

From the plot Fig 2.17 we see that spectrum is discrete indicating that only certain wavelengths can attain optical gain.

\section{Cavity length and number of longitudinal modes in the cavity:}

When $1>>\lambda$ then the spacing between the modes is very small and many modes can exist in the output spectrum. The condition for a single mode, $\mathrm{q}=1$, operation is given by 


$$
1=\lambda / 2 \mathrm{n}
$$

From the above 2.10 we see that the cavity length determines the number of longitudinal modes present in the laser cavity. Shorter cavities allow fewer wavelengths to propagate in the cavity than a longer cavity..

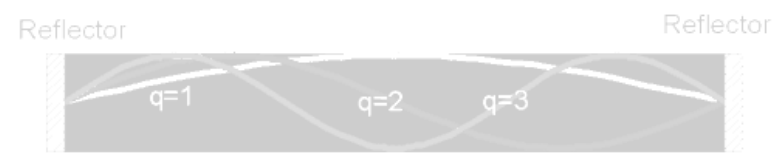

Figure 2.18 [Streetman, 2000]: Wavelengths in a fabry perot cavity

The reflection of these waves at the boundaries gives rise to standing wave distributions in between boundaries these are called transverse modes. Each wavelength can have certain number of transverse modes depending on the dimensions of the cavity. The optical gain at each wavelength is a combination of different modes possible for that wavelength.

\subsubsection{Transverse Modes:}

Due to interference from waves propagating in the cavity there is a standing wave distribution with respect to the dimensions of the cavity in the direction perpendicular to the direction of propagation (across the area of cross section of the cavity) and along the length of the cavity.

These modes are again classified into TE and TM modes depending on the polarization of the electric field with respect to the direction of propagation in the active region.

TE polarization: If the magnetic field has a component in the direction of propagation. TM polarization: If the electric field has a component in the direction of propagation. 


\subsubsection{Infinite three slab waveguide:}

A stripe edge emitting laser diode cavity has a standing wave distribution across the cavity due to the reflections in the direction normal to the direction of propagation. Waves traveling in the other directions attenuate quickly due to the absence of stimulated emission along those directions. Therefore standing waves are not observed in those directions. This pattern is similar to the field distribution in a three slab waveguide. For determining the transverse modes in a laser diode a three slab waveguide model was considered Fig 2.17. The figure indicates that the electric field is restricted between the boundaries in the $\mathrm{y}$-direction but can spread out freely in the $\mathrm{x}$ and $\mathrm{z}$ directions. The boundaries in the $y$ - direction reflect light and hence a standing wave pattern is observed in that direction. In the other directions there is no standing wave pattern due to the absence of boundaries.

The number of transverse modes in an edge emitting laser diode is simply given by solution for three slab waveguide

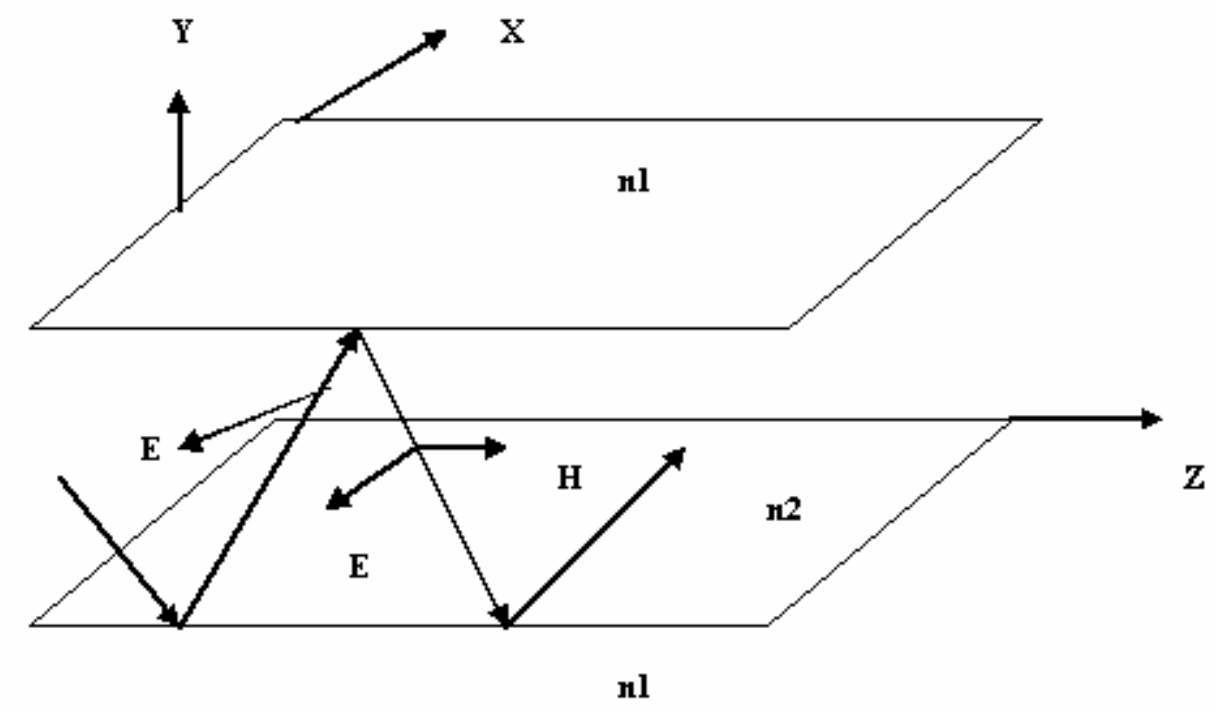

Figure 2.19: 1D- Three slab waveguide- TE mode 
The TE and TM modes are expressed as $\mathrm{TE}_{0 \mathrm{~m}}$ and $\mathrm{TM}_{0 \mathrm{~m}}$ respectively. The subscript $\mathrm{m}$ indicates the number of nodes or zero intensity fields along the direction of confinement for the TE and TM modes. There are no nodes along $\mathrm{x}$ axis which is represented by a subscript with a constant value 0 .

In Fig 2.19 the direction of propagation of the wave is along the z-axis and the polarization to TE we have the magnetic field in the direction of propagation and electric field perpendicular to the direction of propagation.

The number of modes existing in a slab waveguide a function of the normalized frequency parameter, $V$ number, which is given by:

$$
V=\left[\frac{2 \pi a\left(n_{1}^{2}-n_{2}^{2}\right)^{\frac{1}{2}}}{\lambda}\right]
$$

a - thickness of the slab waveguide

$\lambda-$ free space wavelength

$\mathrm{n}_{1}-$ refractive index of core

$\mathrm{n}_{2}-$ refractive index of cladding.

For single mode operation for both TE and TM modes the cutoff condition is $V \leq \pi$ for $\mathrm{a}=0$ the lowest order mode disappears.

A plot of the field distribution with respect to the direction of propagation can be obtained by solving the Maxwell's EM equations subjected to the appropriate boundary conditions. Only the $\mathrm{TE}_{00}$ mode has a field distribution that is close to the gaussian distribution Fig 2.22. The emitted energy is distributed in-between the transverse modes. In order to get a high gain a high current density is required. To achieve lasing at low current densities, if the other modes are suppressed then the quantum wells can placed 
such that they enhance the gain uniformly, and increase the peak value at emission intensities which is not possible with the presence of other modes.

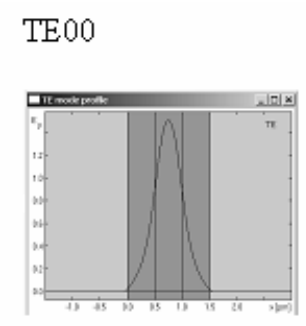

TM00

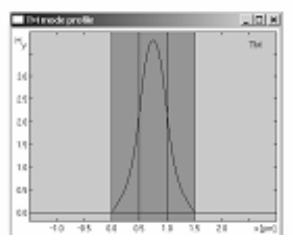

TE01

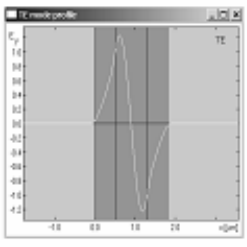

TM01

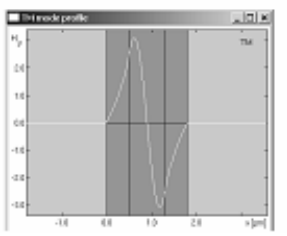

TE02

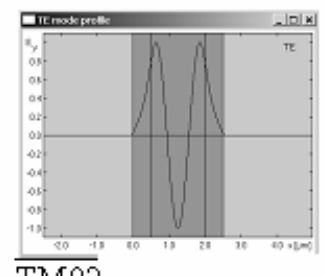

$\overline{\mathrm{TMO} 2}$

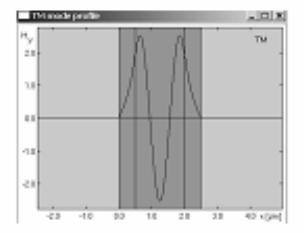

Figure 2.20: TE and TM modes in a three slab waveguide, Intensities of TE and TM profiles for $\mathrm{n} 1=2.5067, \mathrm{n} 2=2.47, \lambda=420 \mathrm{~nm}$. Simulated using online 1D mode solver

It is for this reason single mode operation is desirable. If fewer longitudinal modes exist then the emitted power will be entirely consume by the supported wavelength.

\subsubsection{Single transverse mode condition for both TE and TM modes:}

$$
\text { Thickness } a=\left[\frac{\lambda}{2\left(n_{1}^{2}-n_{2}^{2}\right)^{\frac{1}{2}}}\right]
$$

Multimode condition results when the factor $\mathrm{V}>3.114$. When $\mathrm{V}>10$ the number of modes is approximately given by: $\mathrm{M}=\left[\frac{V^{2}}{2}\right]$.

\subsubsection{Confinement of the electric field to the core $T E_{00}$ mode for our design:}

The more the optical field is confined to the optical guiding region or core the less is the intensity of the evanescent field in the cladding. This implies a high gain in the cavity for a same current density. The extent to which a field is confined to a cavity depends on the 
thickness of the core, the wavelength and the relative refractive index between the core and cladding.

A plot of the confinement of field intensity at the boundary of the core and cladding region(cross over point) as a percentage of maximum field intensity for single mode operation with $\mathrm{n}_{1}=2.5067 \lambda=410 \mathrm{~nm} \mathrm{a}=0.5 \mu \mathrm{m}$ for varying refractive indices, thickness of core is given below Fig 2.21 .
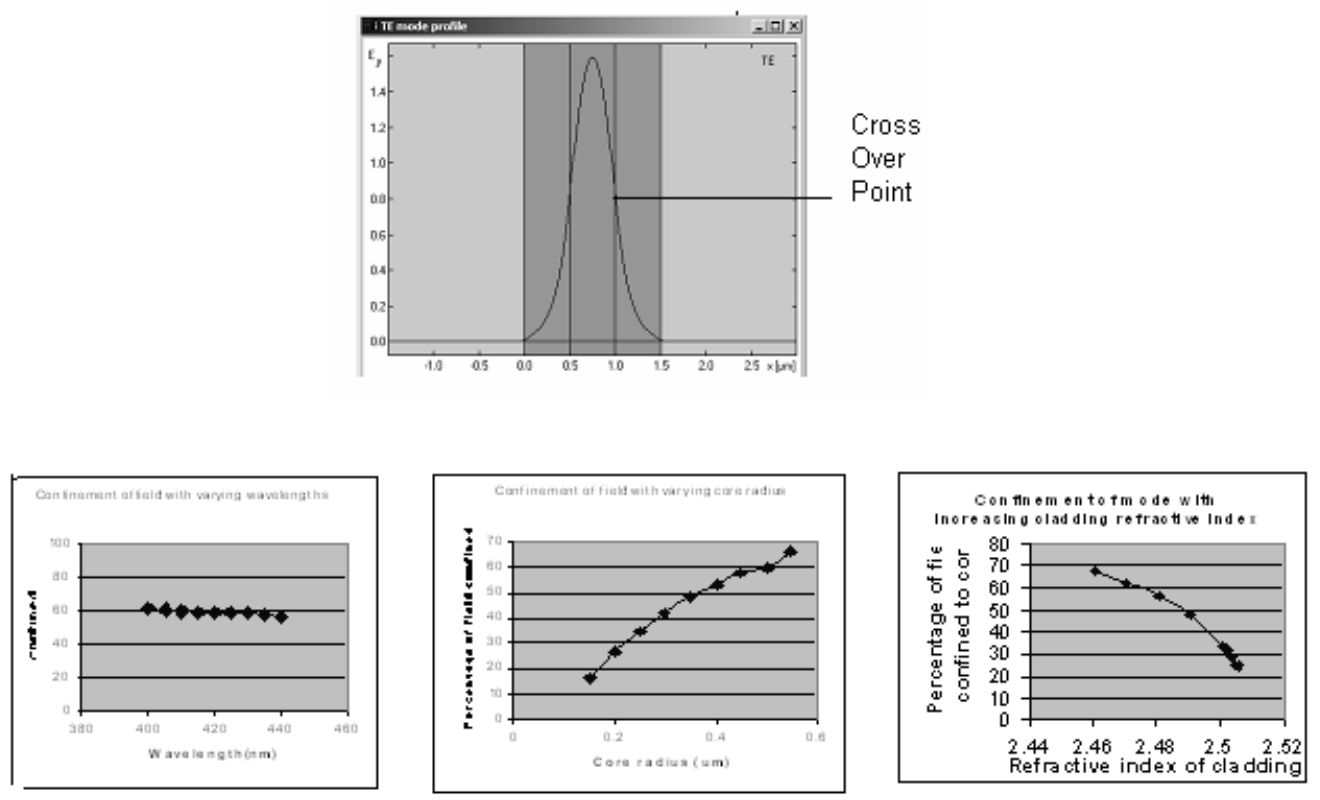

Figure 2.21: Percentage of the optical field confined to the core for varying wavelengths, core thickness and refractive index of cladding $\mathrm{a}=0.5 \mu \mathrm{m} \mathrm{n}_{1}=2.5067 \mathrm{n}_{2}=2.4760 \lambda=$ $410 \mathrm{~nm}$. Simulated using online 1D mode solver

\subsection{Gaussian Beam:}

The electric field distribution of the lowest order mode of laser diode intensity follows a gaussian type distribution. The intensity of the electric field decreases radially when moving away from the center of the axis Fig 2.21. The field distribution is more uniform with increasing distance along the axis. 


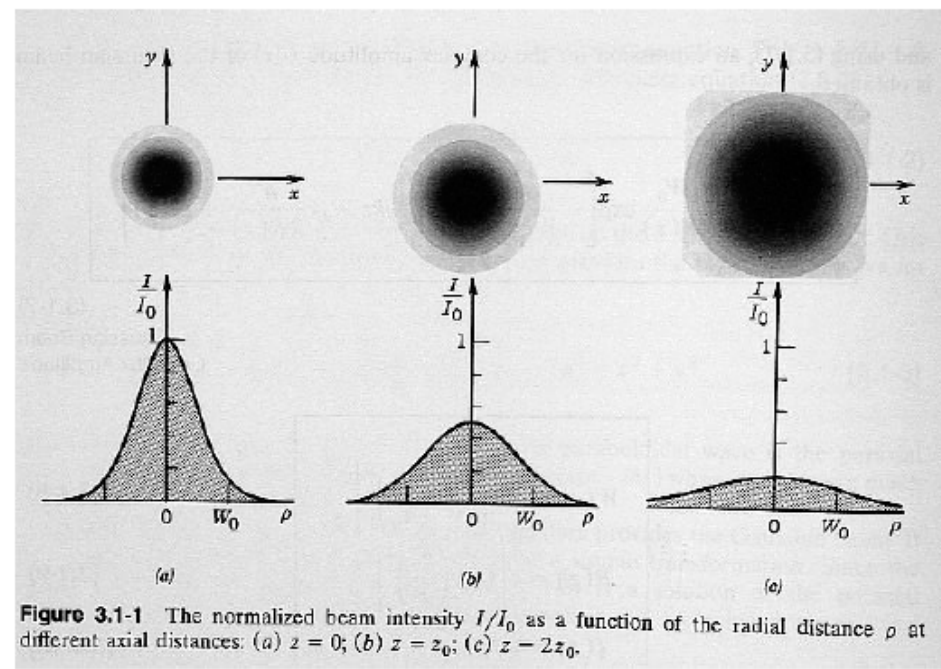

Figure 2.22 [Bahaa, 1991]: Gaussian field distribution outside the laser cavity with increasing radial distance

For a an edge emitting laser diode the output beam spreads elliptically due to diffraction effects at the rectangular cross section edges Fig 2.22.

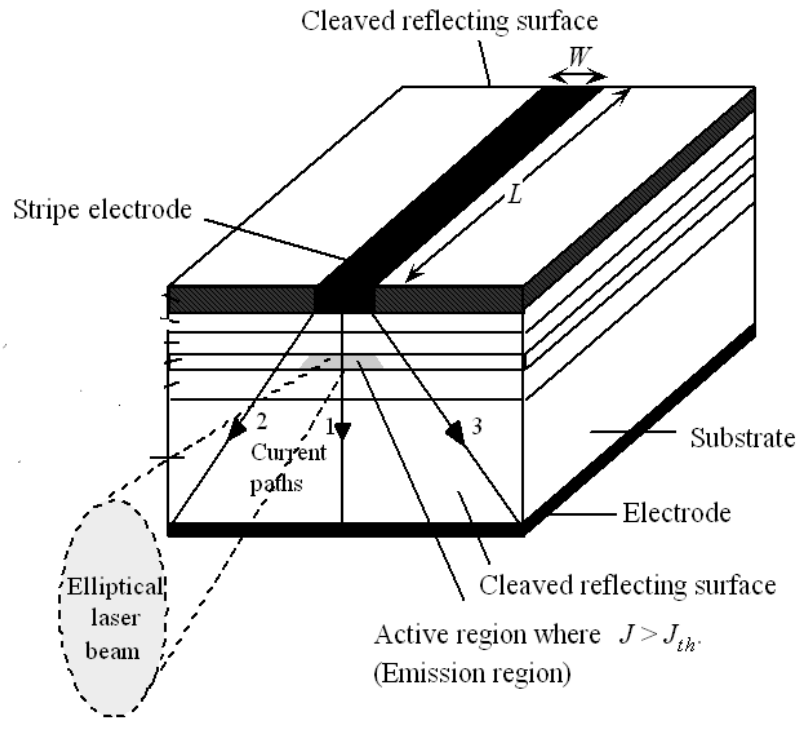

Figure 2.23 [Kasap, 2001]: Typical structure of an edge emitting laser diode 


\subsubsection{Output power vs Current characteristics:}

The current density at which the laser begins to laze is called threshold current. From the plot between output power vs driving current Fig 2.23, we can see that the output power is very low for currents below threshold current indicating a spontaneous emission and for driving currents above the threshold the output power increases exponentially indicating stimulated emission dominates Fig 2.23 .Also the value of the threshold current density increases with increasing temperature of operation. At higher temperatures electrons have sufficient energy to overcome the potential barrier and are therefore not confined to the potential well. Therefore a higher current density is required for laser action.

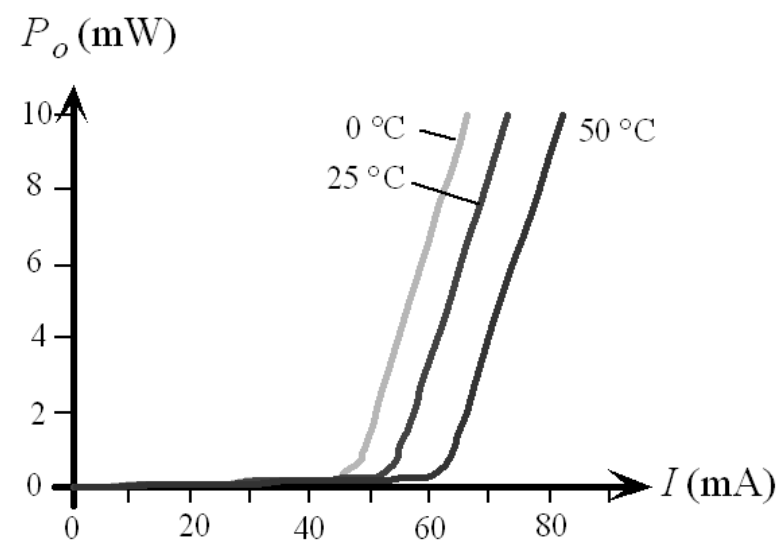

Figure 2.24 [Kasap, 2001]: Output power vs injection current of an edge emitting laser diode

\subsubsection{Applications of semiconductor laser diodes:}

- SLD find applications in laser printing for high resolution printing.

- As the focused laser beams SLD can code a digital signal in forms of pits into a CD.

- The compact size and Gaussian beam emission makes them convenient for integrating into fibers for applications in optical fiber communication.

- High power single mode diode lasers are used in medical applications for retinal and blood vessel surgery. 


\subsubsection{Blue Laser Diodes:}

Shuji Nakamura of Nichia was the first to produce blue light emitting laser using GaN and its alloys. Blue laser diodes emit light in the wavelength of range 440nm- $485 \mathrm{~nm}$. This region of operation has led to applications in optical data storage i.e. in CDs and DVDs and high resolution printing.

\subsubsection{Blue laser diode applications:}

- The short wavelength of blue laser diodes decreases the beam spot size used to etch pits in the optical storage devices like CDs and DVDs this increases the density of data that can be stored on the disc and hence more memory.

- In order to improve the quality and resolution of the images or text printed, printers require the number of dpi (dots per inch) to be high. This can be achieved by using a blue laser diode which has a shorter wavelength when compared to the currently used an infra red beams and hence a smaller beam spot size can be achieved.

\subsubsection{Materials used for blue laser diodes:}

Emission of photons in the blue region requires a material having a bandgap of $2.64 \mathrm{ev}$. This band gap can also be tailored by alloying wide bandgap $(>2.4 \mathrm{ev})$ materials.

\subsubsection{Wide bandgap materials for blue laser diodes:}

The materials under investigation were group III-V, II-V1, and SiC investigation as they possessed sufficient energy gap for emission in the blue region. $\mathrm{SiC}$ due to its indirect band gap has poor internal quantum efficiency and was ruled out for the active region of the laser diode [Troika, 1999]. Other material systems with direct bandgap under consideration were Zone (3.3ev), GaN (3.4ev) [Alivova, 2003] and $\mathrm{ZnSe}(2.7 \mathrm{ev}$ )[Wilmers, 1999]. ZnSe based laser could never be realized commercially due to their short life time [Ueta, 2004] and the future of $\mathrm{ZnO}$ light emitter depends on producing low resistivity p - contacts [Look, 2004]. Experimental results showed that laser diodes fabricated using GaN had high internal quantum efficiency and longer life time inspite of having a high dislocation density. These results led to the commercialization of GaN based blue laser diodes [Nakamura, 1998]. 


\subsection{Problems with GaN:}

Even though GaN has the potential to address critical issues like long life time for commercial purposes exceeding 10000hours of RT CW operation, there are several problems associated with the fabrication of these devices. The main drawback is the crystal quality. The lack of high quality substrates for homoepitaxy or other lattice matched substrates leads to a material with high dislocation density [Monroy, 2003]. Dislocations act as non radiative recombination centers thus reducing the internal quantum efficiency of the material [Lester, 1994]. The other problem is related to the high activation energy of dopants in GaN especially for p-type doping and lack of suitable metallic contacts for the $\mathrm{p}-\mathrm{GaN}$. The above are some of the critical parameters which hinder GaN from operating to its potential.

To overcome these problems to some extent, unique defect reduction substrates like ELOG (Epitaxially laterally overgrown $\mathrm{GaN}$ ) were grown to prevent the propagation of defects into the GaN laser diode during fabrication [Nagahama,2001]. In spite of not finding a metal with proper work function for formation of low- resistivity ohmic contacts to $\mathrm{p}-\mathrm{GaN}$ deposition of metals like $\mathrm{Au} / \mathrm{Ni}, \mathrm{Ti} / \mathrm{Pt} / \mathrm{Au}$, or $\mathrm{Au} / \mathrm{Ni} / \mathrm{Au}$ on $\mathrm{p}-\mathrm{GaN}$ along with an additional step of thermal annealing under various test conditions produced low resistivity ohmic contacts to p-GaN [Maruyama, 2001], [Zhou, 2000], [Narayan, 2002].

\subsubsection{Group III-V based InGaN/AlGaN Multi Quantum Well laser diodes:}

The first edge emitting InGaN laser diode was demonstrated by Nakamura. The life time of the laser diode was a few hours and it was operated under pulsed and CW conditions at RT [Nakamura, 1996]. The laser diode structure was a separate confinement heterostructure $(\mathrm{SCH})$ with a multi quantum well (MQW) InGaN as the active region. $\mathrm{GaN}$ was the optical guiding region with an $\mathrm{AlGaN}$ layer as the cladding. The emission spectrum was in the violet region centered at $417 \mathrm{~nm}$. 


\subsection{Bandgap properties and Optical properties of InGaN:}

The bandgap of $\mathrm{InGaN}$ is given by:

$\mathrm{Eg}\left(\operatorname{In}_{\mathrm{x}} \mathrm{Ga}_{1-\mathrm{x}} \mathrm{N}\right)[\mathrm{eV}]=\mathrm{Eg}(\operatorname{InN}) \mathrm{x}+\mathrm{Eg}(\mathrm{GaN})(1-\mathrm{x})-\mathrm{bx}(1-\mathrm{x})$

[Binder , 2000]

The bowing parameter is given from the plots Fig 2.26

From the above the bandgap of InGaN can be varied from $1.89 \mathrm{ev}$ to $0.8 \mathrm{ev}$ (the bandgap of $\mathrm{InN}$ is still being debated) for an indium mole fraction of 1 to $3.42 \mathrm{ev}$ for an indium mole fraction of 0 Fig 2.24. This covers the emission in deep UV region to infrared in the visible spectrum. This makes the InGaN system a potential candidate for projecting full color images at low powers.

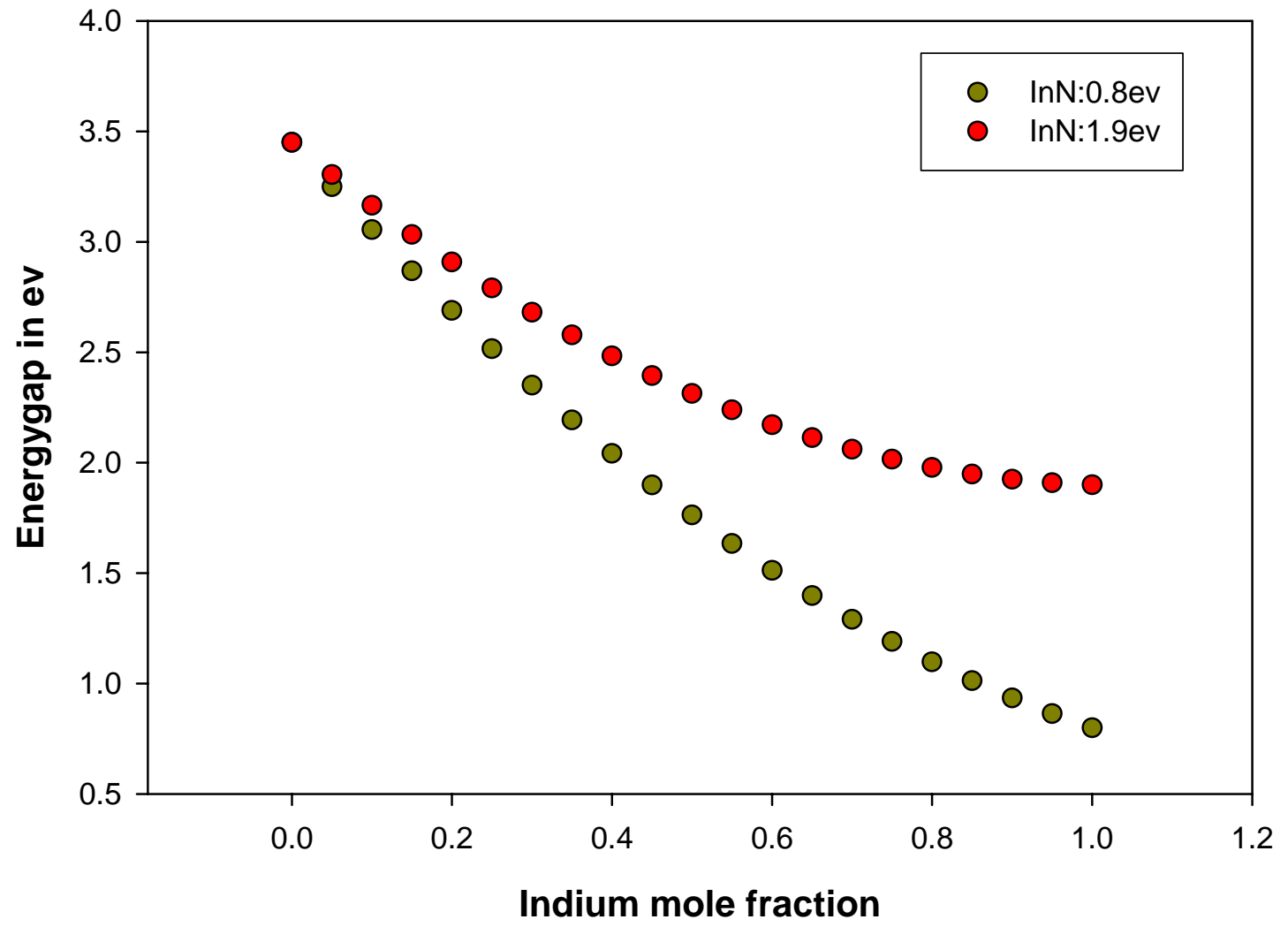

Figure 2.25: Energy gap of InGaN as function of indium mole fraction

In addition to the variation of indium mole fraction and quantum well thickness, the emission wavelength of an $\mathrm{InGaN}$ quantum well is also dependent on the growth 
conditions. This variation of growth conditions can lead to effects like strong piezoelectric effect in InGaN quantum well, formation of indium rich quantum dots and variation of bandgap bowing with changing indium mole fraction.

\subsection{Issues related to the indium phase segregation - quantum dots:}

The low miscibility between $\mathrm{InN}$ and $\mathrm{GaN}$ can lead to indium phase segregation leading to the formation of indium rich quantum dots. Carriers are trapped in these deep localized states giving out emissions at lower wavelengths [Wang, 1999], [Krestnikov, 2002].

\subsection{Piezoelectric effect:}

It was reported that the piezo electric field generated in the quantum wells effects the emission energy [Martin, 2002]. The discrepancy in the data of the predicted and experimental emission wavelengths of the quantum well Fig 3.4 may be well be an effect of the piezo electric effect.

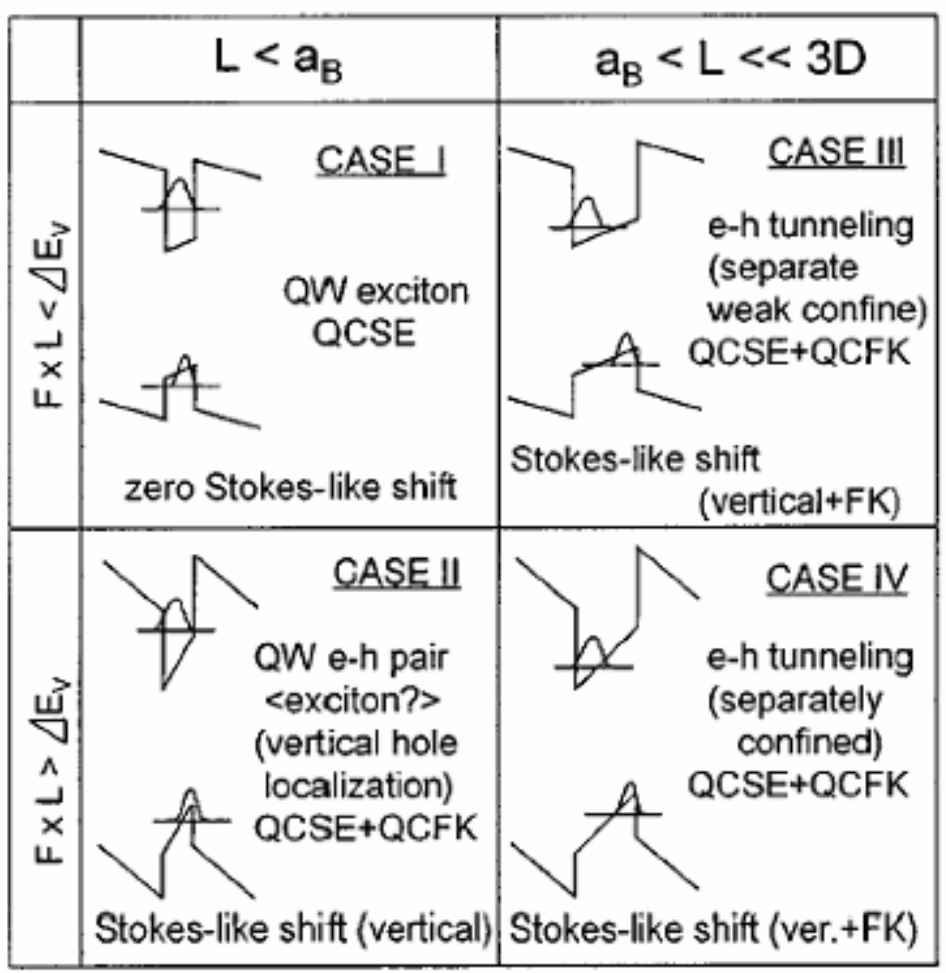

Figure 2.26 [Chichibu, 1998]: Piezoelectric field in an InGaN quantum well 


\subsection{Variation of bowing parameter with Indium mole fraction:}

First principle calculations have indicated that the band gap bowing varies with fluctuation in the indium mole fraction [Ferhat, 2001]. The plots Fig 2.26 reveal that the bowing parameter decreases with increasing indium mole fraction.
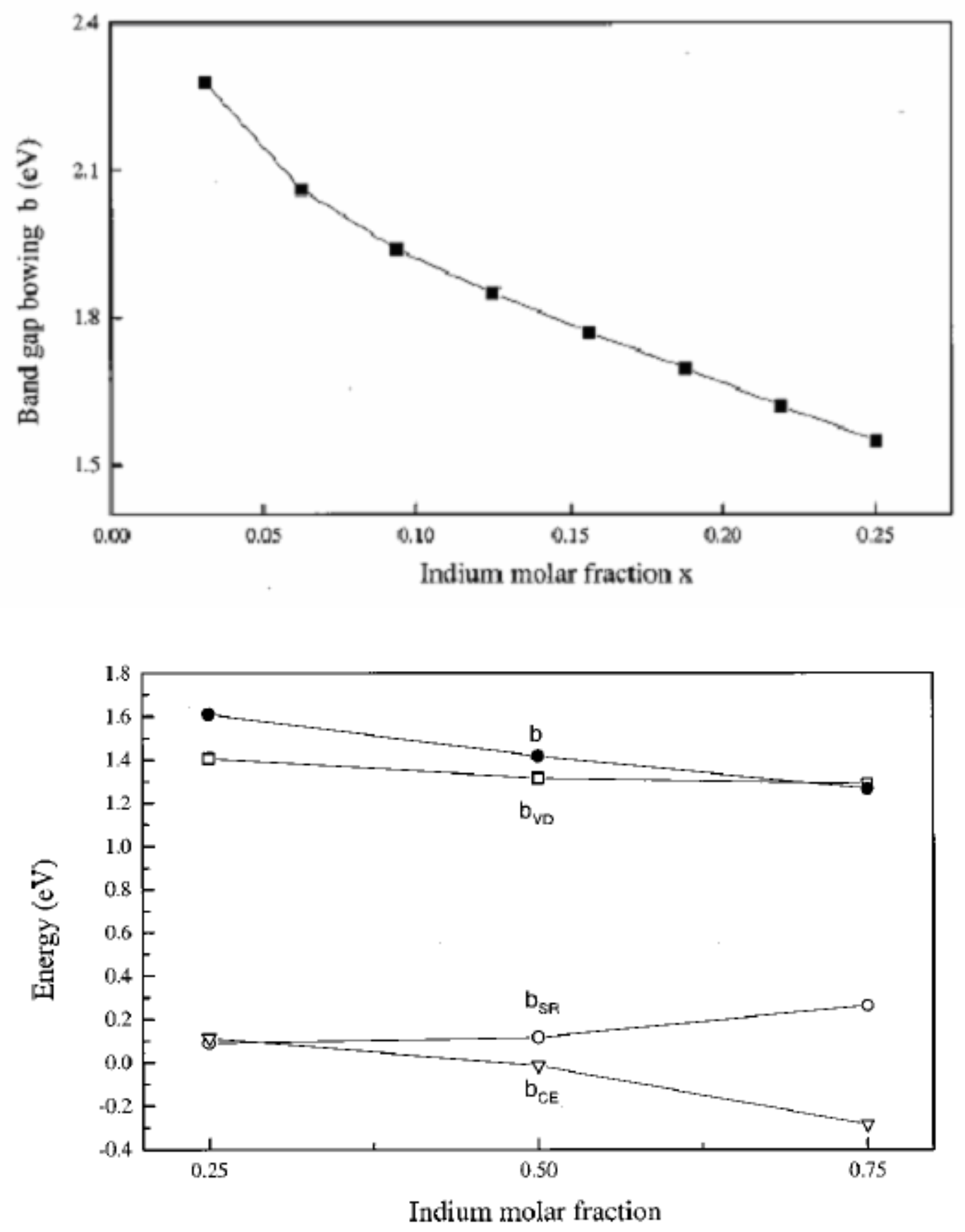

Figure 2.27 [Ferhat, 2001] :Variation of InGaN bandgap bowing as a function of Indium mole fraction, Bandgap bowing is indicated by dark circles 


\subsection{Bandgap and Optical properties of AlGaN:}

The bandgap of $\mathrm{AlGaN}$ is given by:

$\operatorname{Eg}\left(\mathrm{Al}_{\mathrm{x}} \mathrm{Ga}_{1-\mathrm{x}} \mathrm{N}\right)[\mathrm{eV}]=\operatorname{Eg}(\mathrm{AlN}) \mathrm{x}+\operatorname{Eg}(\mathrm{GaN})(1-\mathrm{x})-\mathrm{bx}(1-\mathrm{x})$

$\operatorname{Eg}(\mathrm{AlN})=6.28 \mathrm{ev}, \operatorname{Eg}(\mathrm{GaN})$ wurtzite structure $=3.42$, Bowing parameter $\mathrm{b}=-0.36 \mathrm{ev}$.

[ Umit Ozgur,2001]

The refractive index of $\mathrm{AlGaN}$ is given by:

$$
n(\lambda)^{2}=1+\frac{A_{o} \lambda^{2}}{\lambda^{2}-\lambda_{o}^{2}}
$$

[Ozgur, 2001]

$A_{o}\left(E_{g}\right)=B_{0}+B_{1} E_{g}+B_{2} E_{g}^{2}, \lambda_{0}\left(E_{g}\right)=C_{0}+C_{1} E_{g}+C_{2} E_{g}^{2}$

Values of $\mathrm{B}_{0}, \mathrm{~B}_{1}, \mathrm{~B}_{2}, \mathrm{C}_{0}, \mathrm{C}_{1}, \mathrm{C}_{2}$ depend on parallel or perpendicular polarization to the $\mathrm{c}-$ axis.

The refractive index of $\mathrm{AlGaN}$ decreases with increasing $\mathrm{Al}$ mole fraction, with the refractive index of GaN being 2.5067.

For efficient confinement of optical field in the SCH structure the refractive index difference between core and cladding i.e. GaN and $\mathrm{AlGaN}$ respectively must be very high. This requires a very high concentration of $\mathrm{Al}$ in $\mathrm{AlGaN}$.

\subsection{Doping problems with p-AlGaN:}

The difficulty in obtaining sufficient hole conductivity in wide-bandgap AlGaN is a problem that essentially originates from the deep Mg-acceptor level of $\mathrm{Mg}$-doped $\mathrm{AlGaN}$. Heavy Mg-doping of around $10^{20} \sim 10^{21} \mathrm{~cm}^{-3}$ is required in order to obtain adequate hole conductivity due to the low activation ratio of holes in high Al-content AlGaN. Such heavy Mg-doping causes the degradation of the AlGaN crystalline quality [Obata, 2004]. Achieving hole concentration of $10^{18} \mathrm{~cm}^{-3}$ at an $\mathrm{Al}$ mole fraction of 0.32 is now a possibility Fig2.27. But this mole fraction is not sufficient for confining the electrical field completely to the core. 


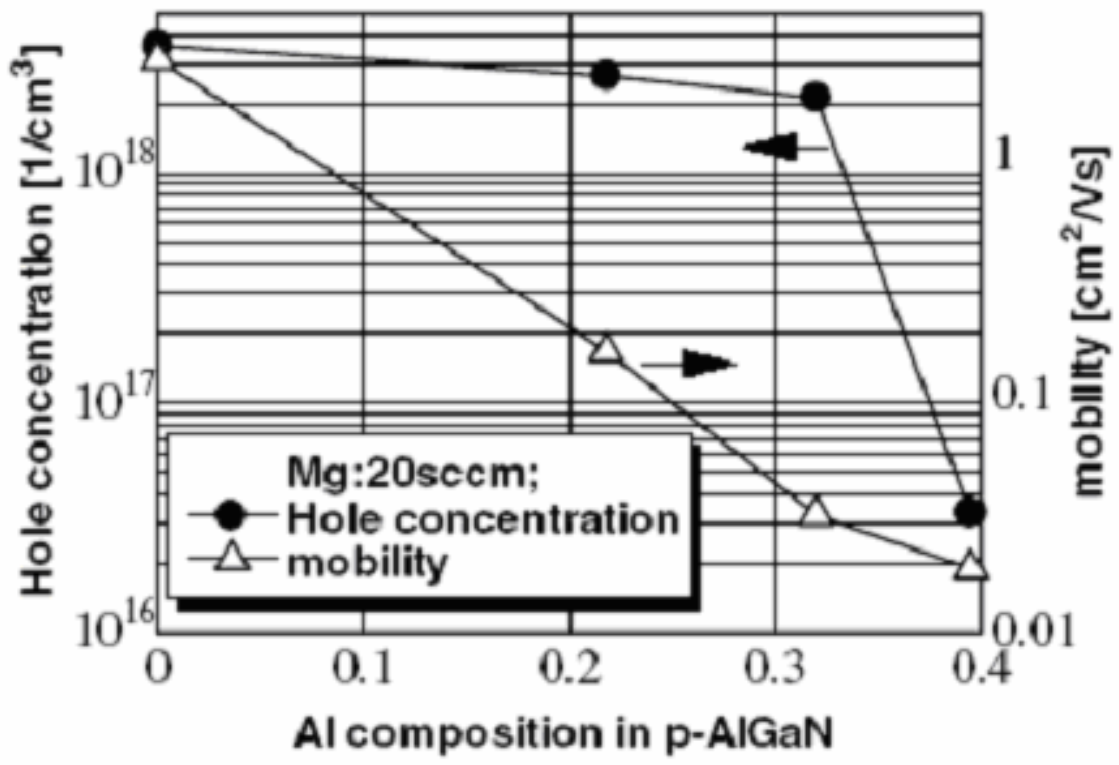

Figure 2.28 [Obata, 2004]: Variation of hole concentration in AlGaN as a function of Al mole fraction

\subsection{Structure of InGaN laser diode Fig 2.28:}

Laser diodes with different design layout have been fabricated and most of the designs reported were $\mathrm{SCH}$ devices which had multiple quantum wells as the active region with $\mathrm{GaN}$ as the optical guiding region and $\mathrm{AlGaN}$ layer as the cladding region. Additionally there is an electron blocking p-AlGaN layer which prevented overflow of electrons. Laser diodes with different emission wavelengths, efficiencies, output powers and threshold currents were reported by varying the properties of each of these layers. In some cases different substrates like ELOG on sapphire were tried out to reduce the propagation of defects into active region during growth.

The electron blocking layer $\mathrm{p}$-AlGaN creates a barrier for the holes in the p-side to move freely into the quantum well. Structures have been reported without the use of the electron blocking. 


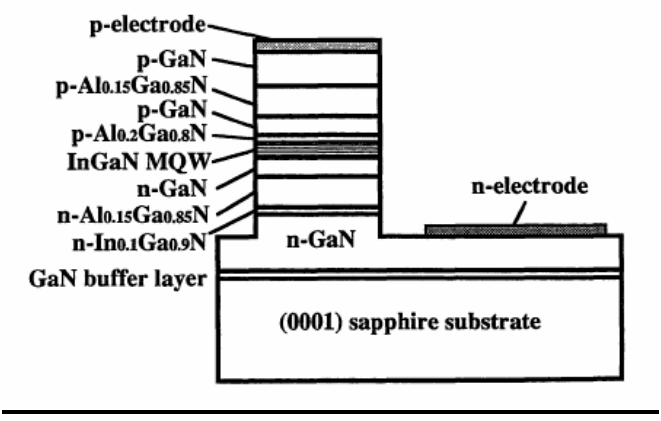

Figure 2.29 [Nakamura, 1996]: Structure of blue laser diode fabricated by Shuji Nakamura

Fujitsu 3 (1999)

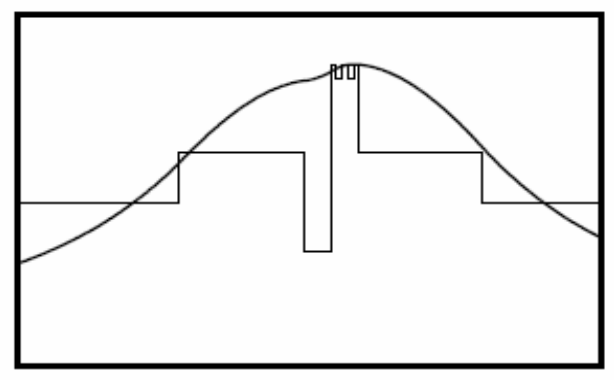

Nichia 1 (1996)

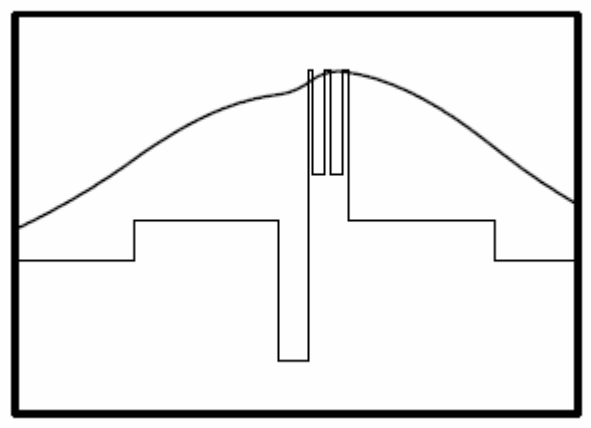

Nichia 2 (1997)

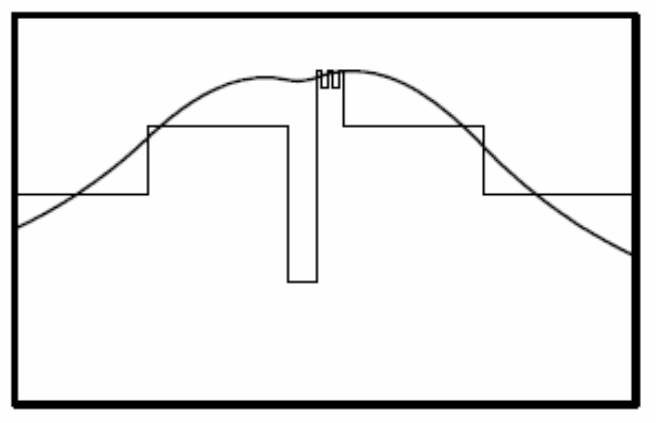



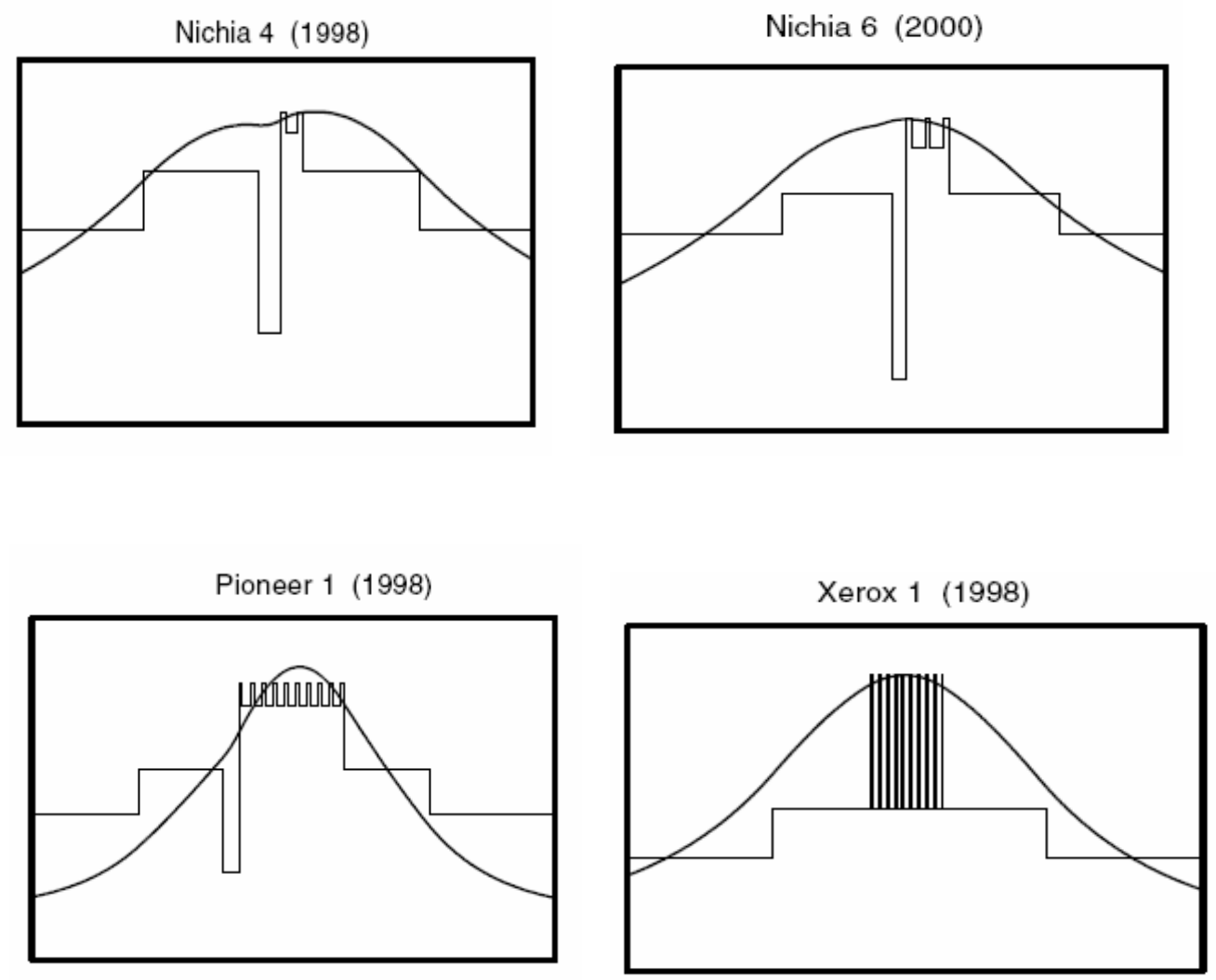

Figure 2.30 [Czyszanowski1, 2001]: Refractive index profiles of advanced nitride laser diodes with electric field intensity in the optical guiding region

In order to prevent cracking of $\mathrm{AlGaN}$ cladding layer $\mathrm{AlGaN} / \mathrm{GaN}$ modulation doped strained layer superlattice was used for some structures [Hansen, 1999]. 


\section{PROPOSED STRUCTURE FOR BLUE LASER DIODE}

3.1. Overview: This chapter discusses about the design of a separate confinement heterostructure for a laser diode emitting blue light. Further the problems with typical InGaN quantum well laser diodes are studied and solutions are proposed to overcome these problems.

\subsection{Design of MQW InGaN blue laser diode:}

Operation of a laser diode at low bias voltage is one of the major requirements for successful commercialization of a laser diode. Efficient confinement of the electric field to the optical gain region minimizes the optical losses and this requires a high refractive index contrast between the core and the cladding region and thus a high Al mole fraction in the cladding region. But there are conductivity problems associated with high $\mathrm{Al}$ mole fractions in n-AlGaN [Bradley, 2003] [Zhu, 2004] and p-AlGaN [Obata, 2004]. One of the major factors that prevent the operation of a blue laser diode at low bias voltage is the doping problem in $\mathrm{n}-\mathrm{AlGaN}$ and $\mathrm{p}-\mathrm{AlGaN}$ cladding layers at high $\mathrm{Al}$ mole fraction. At high $\mathrm{Al}$ mole fractions it is difficult to achieve high conductivity in $\mathrm{AlGaN}$ cladding layers .The low conductivity of the AlGaN layers increases the series resistance of the device and this requires applying a large bias to get the required carrier density for lasing action. This reduces the external quantum efficiency of the laser diode.

The active region consisting of InGaN quantum wells also plays an important role in efficient operation of the laser diode. The active region is responsible for confining the carriers in the active region through finite potential InGaN quantum wells. The small conduction band offset [Blood, 2000] causes electron current overflow which leads to improper confinement of carriers in the quantum well. Therefore a higher carrier density is required to achieve lasing.

In this work we modeled an $\mathrm{SCH}$ structure with $\mathrm{InGaN} \mathrm{MQW} \mathrm{as} \mathrm{the} \mathrm{active} \mathrm{region,} \mathrm{GaN}$ as optical guiding region and $\mathrm{AlGaN}$ as the cladding region for emission wavelength in 
the range of $440 \mathrm{~nm}-485 \mathrm{~nm}$. Alternate structures are proposed for the active region QW and claddings regions that may overcome the above stated drawbacks.

\subsubsection{Designing InGaN MQW:}

When the de broglie wavelength of the carriers in the active region of a DHLD is approximately less than or equal to the thickness of the active region, effects not typical of the bulk material known as quantum size effects occur\{Holyank,1980]. Under such conditions are not scattered sufficiently to thermalize from the band edge of the confinement layers to the lower confined - particle states of the quantum well. As such the carriers remain at higher energy levels and have high probability of escaping from the potential well. In this situation laser operation is not possible at lower confined states of the quantum well for low current densities. However if a quantum well is coupled to another via a barrier then the all the excess carriers at higher energy levels are coupled to the adjacent quantum well through the process of quantum tunneling [Holonyak, 1980]. Multiple quantum wells are used to increase the amount of power emitted by the laser diode as the power generated by a single quantum well is limited by the saturation effects. Based on the above, a two quantum well laser diode should have a lower threshold current when compared to a single quantum well laser diode.

\subsubsection{Emission of blue wavelength $(440 \mathrm{~nm}-480 \mathrm{~nm})$ :}

The emission in a quantum well is dominated by the difference in energy levels between the lowest discrete energy level in the conduction band and the highest discrete energy level in the valence band. The discrete energy levels in the conduction and valence band are given are given by solving the Schrödinger time independent wave equation for both the potential wells in the conduction band and valence band respectively (Appendix A). As most of the carriers settle down into the lowest energy level the emission energy is dominated by lower energy levels. The emission wavelength is given by: 


$$
\lambda=\frac{c h}{E_{c 1}-E_{v 1}}
$$

$E_{c 1}$ and $E_{v 1}$ and lowest and highest energy levels in the conduction and valence band of the quantum well respectively Fig 3.1.

c- Velocity of light in free space.

h- Planks constant.

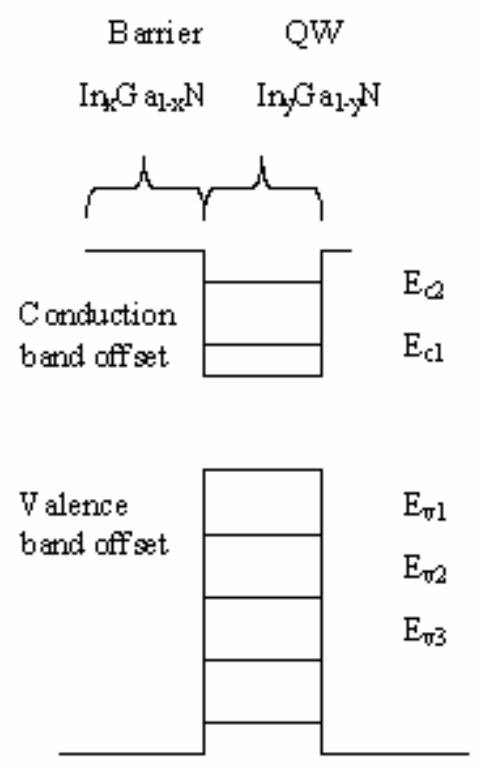

Figure 3.1: Energy levels in a quantum well

From the figure we observe that the emission wavelength is dependent on the position of the energy levels (Ec1 and Ev1) relative to the band edge. This relative position is a function of indium mole fraction in the barrier, the quantum and the quantum well thickness Fig 3.2. 


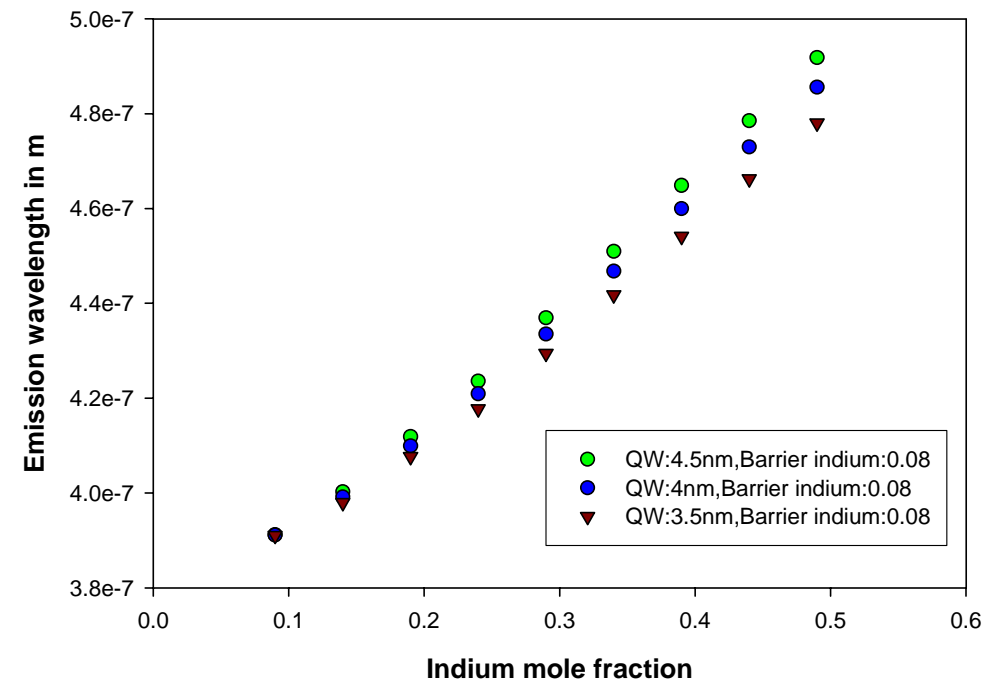

Figure 3.2 Appendix C: Emission wavelength for InN bandgap of 1.9ev

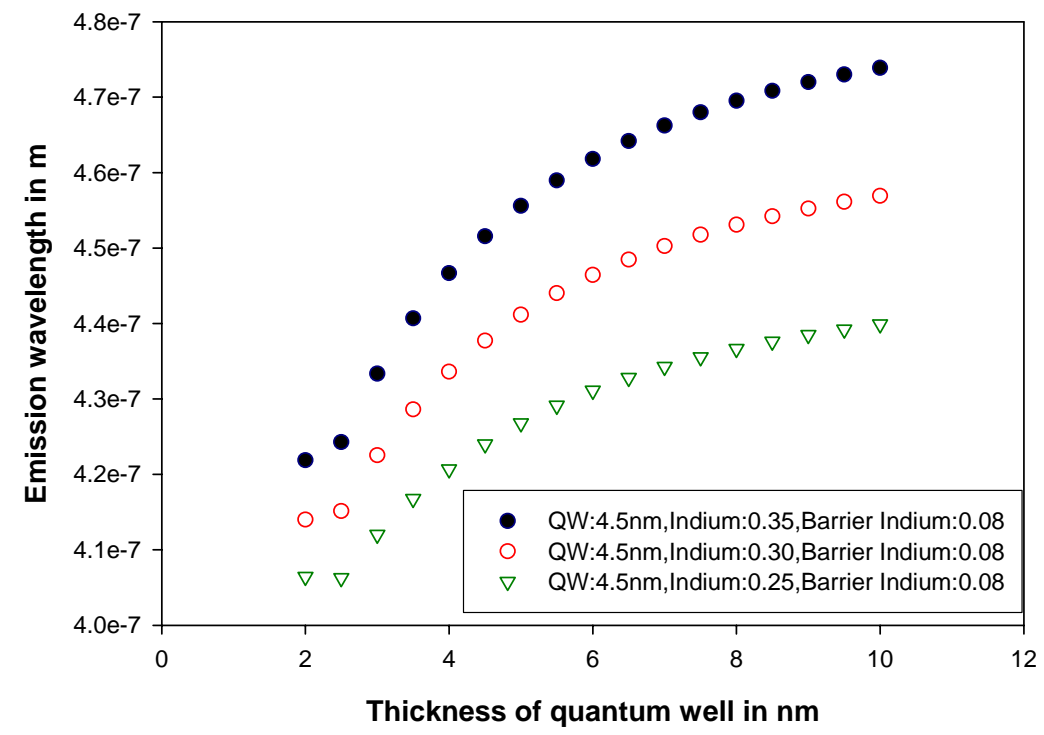

Figure 3.3 Appendix C: Emission wavelength for InN bandgap of 1.9ev

From the plots we see that the same emission wavelength can be tailored by using different combinations of well thickness and indium mole fraction in the quantum well. As mentioned in the previous chapters a higher indium mole fraction in quantum wells 
and thicker quantum wells have their own disadvantages, so a trade off must exist between the indium mole fraction and the well thickness for producing the desired wavelength.

Comparison of theoretical calculated emission wavelengths and experimental data collected from journals for laser diodes grown on MOCVD .Laser diodes operated at RT.

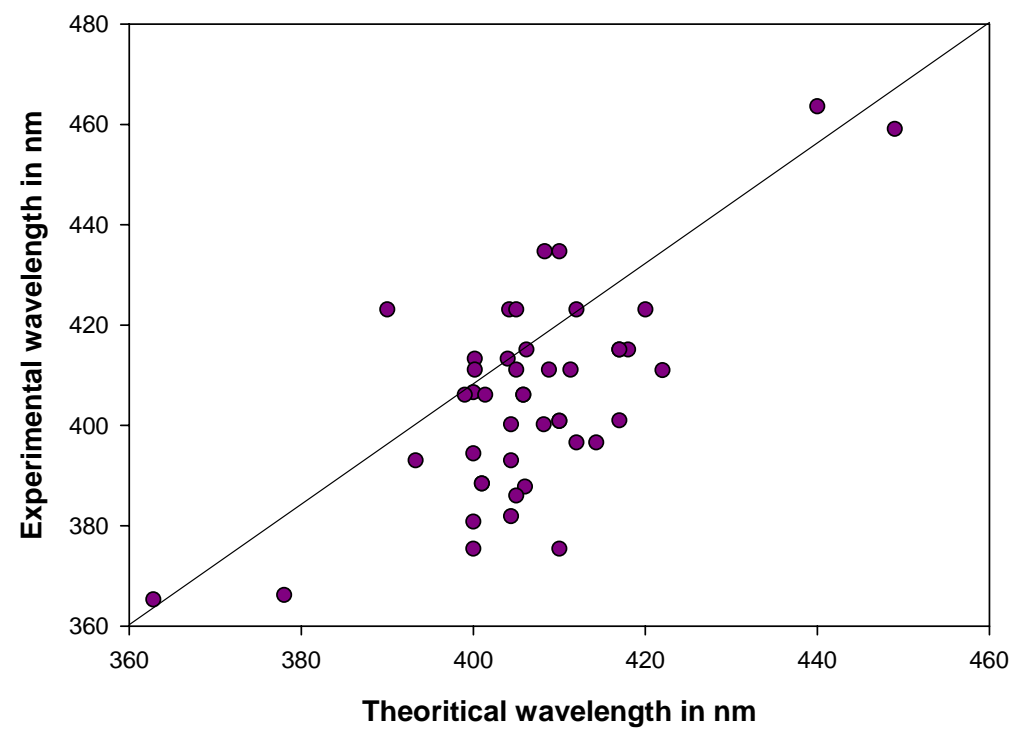

Figure 3.4: Theoretical vs experimental data collected form journals for InGaN laser diodes fabricated on MOCVD and operated at RT

From Fig 3.4 we observe that the predicted and experimental data do not match. This variation help us to understand the growth conditions needed for fabricating laser diodes whose emission wavelengths are predictable.

\subsubsection{Designing GaN Optical guiding region \& AIGaN Cladding region:}

It is assumed that the total thickness of the active region for a two quantum well structure with quantum well width of $3 \mathrm{~nm}$ and barrier thickness of $7 \mathrm{~nm}$, which is very small when compared to the wavelength of operation, does not affect the modes existing in the optical guiding region. The transverse modes existing in the laser diode are determined mainly on the thickness by GaN optical gain region and cladding region refractive index.

For single transverse mode operation the thickness of optical guiding region is given by 


$$
a=\left[\frac{\lambda}{2\left(n_{1}^{2}-n_{2}^{2}\right)^{\frac{1}{2}}}\right]
$$

For operation at wavelength of $450 \mathrm{~nm}$ and with an $\mathrm{Al}$ mole fraction of 0.32 for the cladding region.

Refractive index of AlGaN: 2.278349 at $\lambda=450 \mathrm{~nm}$

$$
n(\lambda)^{2}=1+\frac{A_{o} \lambda^{2}}{\lambda^{2}-\lambda_{o}^{2}}
$$

[Ozgur, 2001]

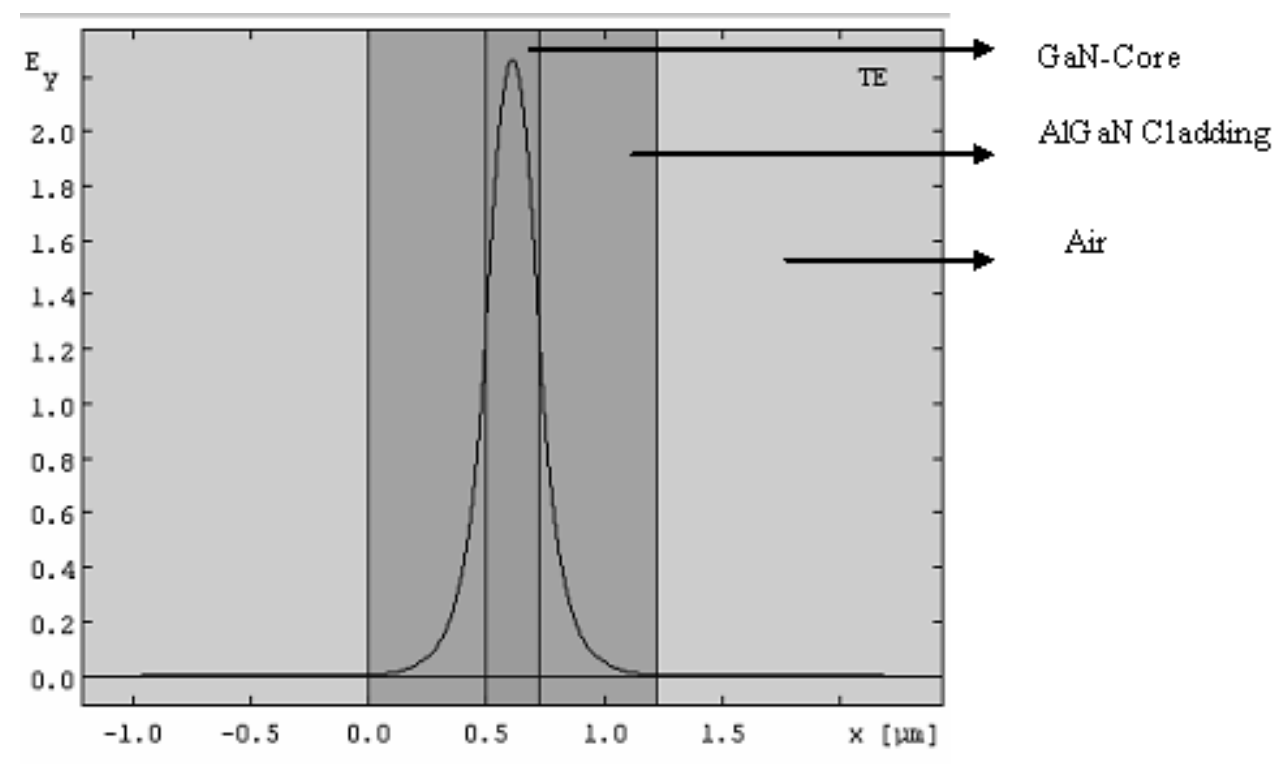

Figure 3.5: Electric field intensity in the optical guiding region and cladding region Simulated using online 1D mode solver Thickness of Optical guiding region is: $0.22 \mu \mathrm{m}$

From the plot we see that the light is not confined completely to the optical guiding region. The field which spreads into the cladding is the evanescent field, which represents loss. In order to compensate for this loss the carrier density needs to be increased to generate more power thus increasing the threshold carrier density of the laser diode. 


\subsubsection{Proposed structures for the active region quantum wells:}

\subsubsection{Graded active region:}

If the thickness of the quantum wells is sufficiently large when compared to the scattering length of the carriers then a large number of carriers thermalize easily to the lower energy levels. When the InGaN quantum well is of thickness comparable to the scattering length of the carriers the carriers do not thermalize sufficiently to settle down at lower energy states. In order to increase the fraction of carriers settling down at lower energy states a higher carrier density is needed. This implies an increase in the threshold current density of the laser diode. Multiple quantum wells solve this problem to some extent. Coupling of a quantum via a thin barrier to another barrier increases the effective length of the active region. The carriers at higher energy levels of the first quantum well have enough distance to travel to thermalize to the lower energy states of the second quantum well. The carriers that settle down in the other quantum well contribute to the net optical gain. This increased optical gain for the same carrier density overcomes the losses in the cavity at lower threshold current densities when compared to a single quantum well structure. But multiple quantum well structures of InGaN suffer from inhomogeneous carrier distribution Fig 3.6. This non uniform distribution of carriers in the quantum wells leads to stimulated emission in quantum wells close to the p-region and absorption in quantum wells away from the p-region and close to the n-region. This occurs due to the low mobility of holes [Domen, 1998]. 


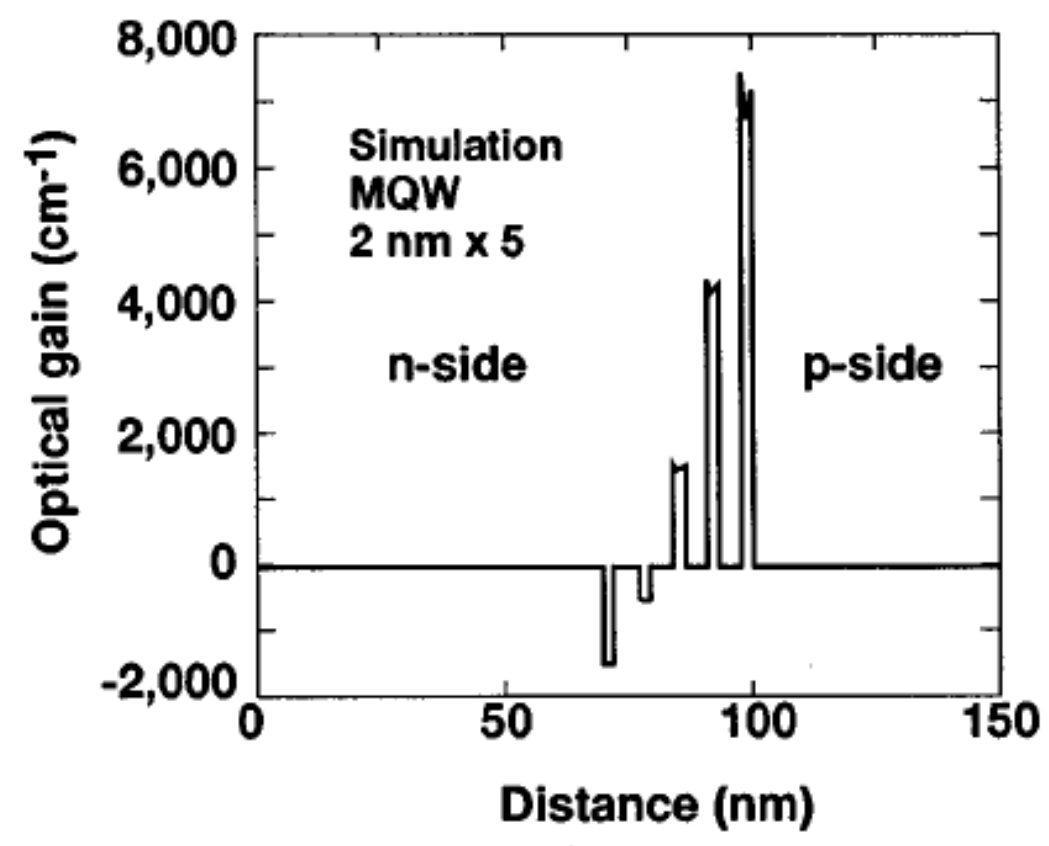

Figure 3.6 [Domen, 1998]: Stimulated emission and absorption in a multiple InGaN quantum well

For the conduction band, increasing the quantum well thickness of the well close to the $\mathrm{n}$ side increases the number of carriers settling down at lower energy states and gradually reducing the thickness of subsequent quantum wells towards to p-side reduces the number of carriers settling down in these wells at lower energy states, when compared to the conventional quantum well structure.

To achieve the same emission wavelength in the laser the Indium concentration of the successive wells has to be varied. Schrödinger time independent wave equation helps us to determine the indium concentration of successive quantum wells.

For understanding the consequences of such a structure we considered the effective mass of carriers as a function of indium mole fraction. The effective mass of carriers gives a measure of their mobility. Higher effective mass means a low mobility and low scattering length of the carriers. In InGaN quantum wells the mobility of holes and electrons is given by: 
$m_{e}=0.15 m_{0} 1-x 0.051 m_{0}$ - Effective mass of electrons.

$m_{h h}=1.449 m_{0}-x 0.146 m_{0}$ - Effective mass of heavy holes.

$m_{l h}=0.157 m_{0}-x 0.104 m_{0}$ - Effective mass of light holes.

$x$ - Indium mole fraction.

$m_{0}$ - Free mass of electron.

[Kuo, 2004]

From the values the effective mass of electrons, light holes and heavy holes in $\mathrm{GaN}$ is higher than that of the carriers in InN. Based on this with an increase in the indium mole fraction the effective masses of electrons and holes in the quantum well decreases Fig 3.7, 3.8 increasing their mobility.

From the above we can infer that to get a uniform distribution of carriers in the quantum wells if the effective mass of the electrons in the quantum wells close to the $\mathrm{n}$ - side is increased and the effective mass of holes in the quantum wells is reduced then we can have a uniformity in the distribution of carriers across the active region.

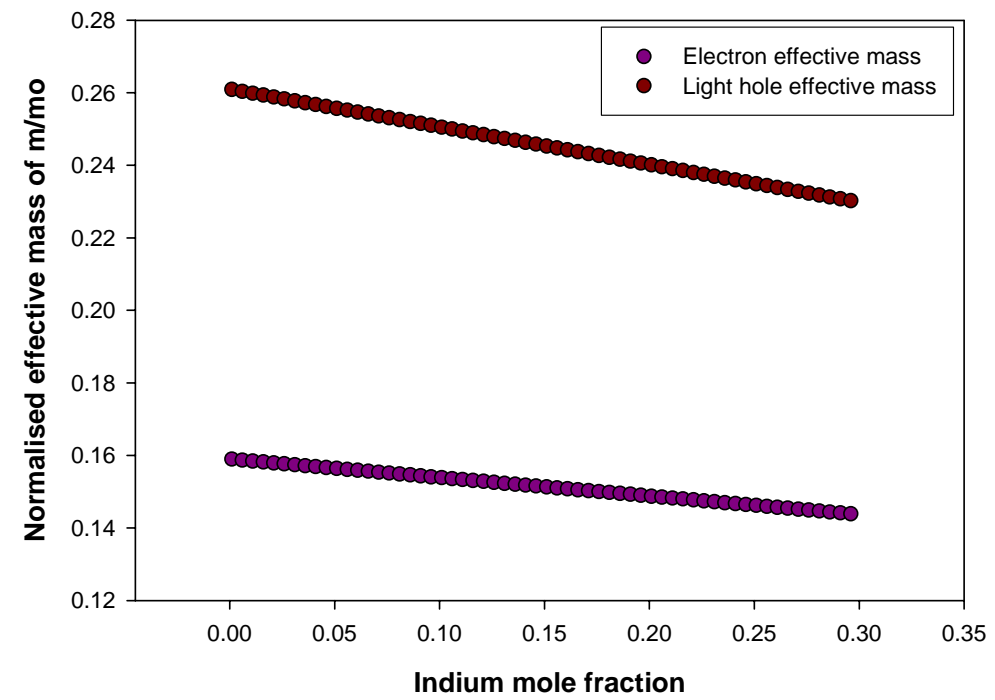

Figure 3.7: Effective mass of electrons and light holes as a function of indium mole fraction 


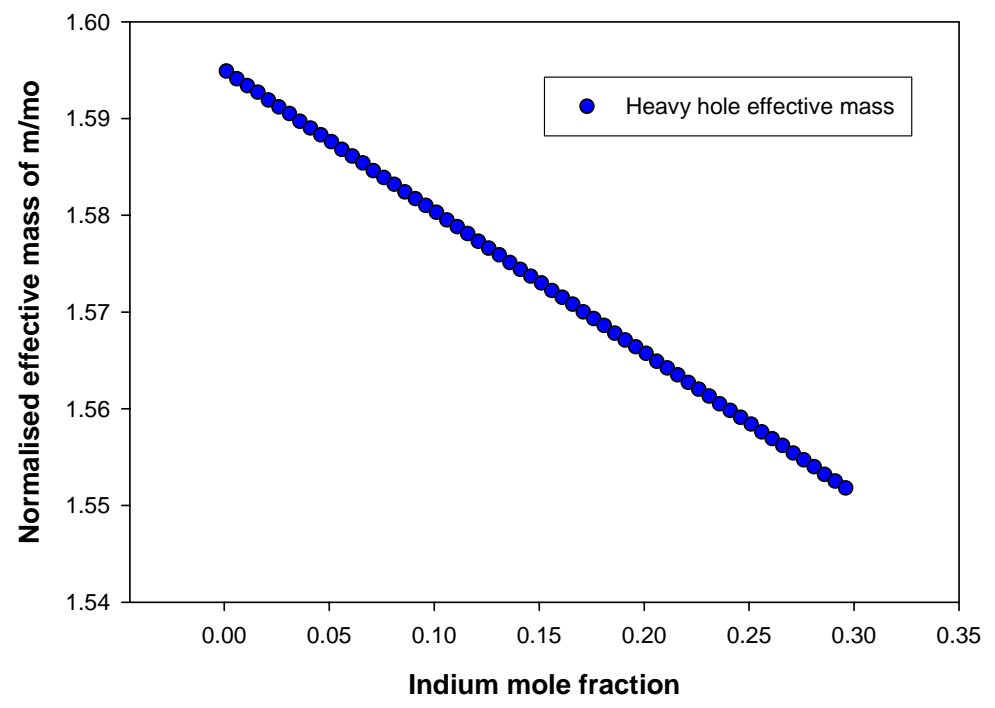

Figure 3.8: Effective mass of heavy electrons as a function of indium mole fraction Graded active region structure

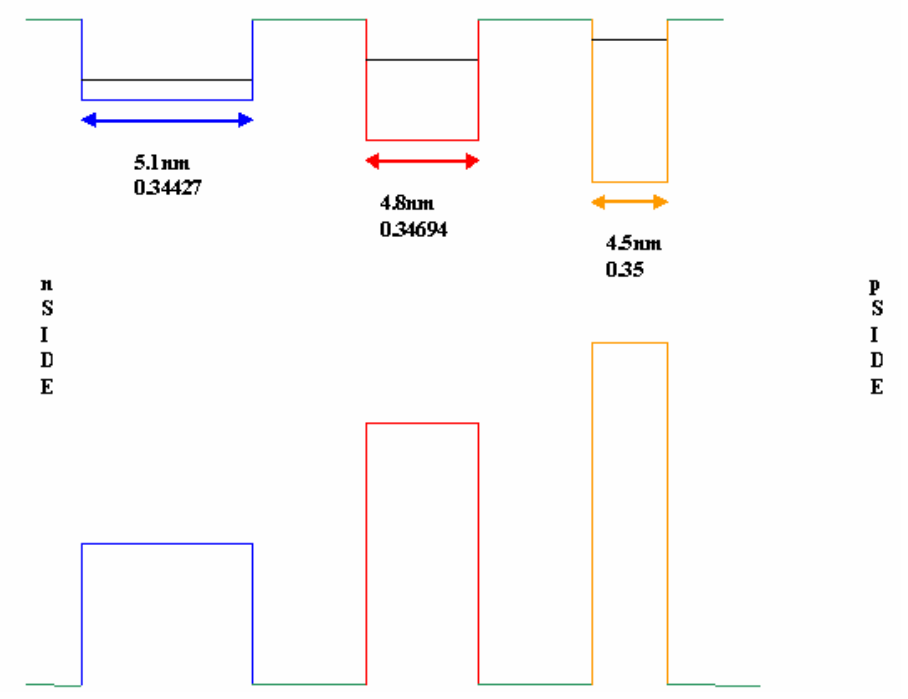

Figure 3.9 Appendix A: Variation of well thickness and indium mole fraction in the active region for an emission wavelength of $450 \mathrm{~nm}$ 
Consequences of this structure Fig 3.9 are that the density of states in the first quantum well to the $n$-side is higher that the subsequent quantum wells. This density of states reduces gradually from the quantum well close to $\mathrm{n}$-side to the successive quantum wells. More carriers are therefore expected to settle down in first quantum well when compared to the conventional structure.

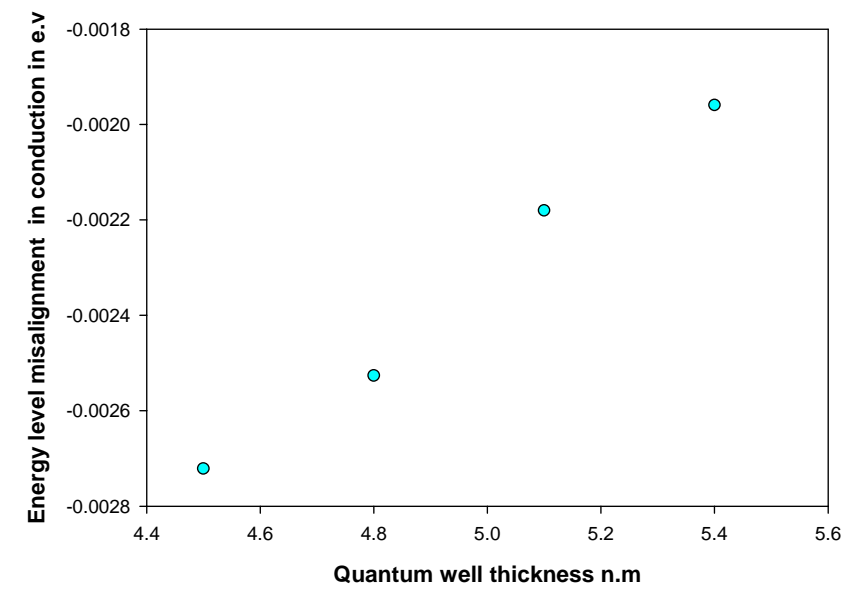

Figure 3.10: Alignment of discrete energy levels in the structure Fig 3.7 conduction band for InN bandgap of 1.9e.v

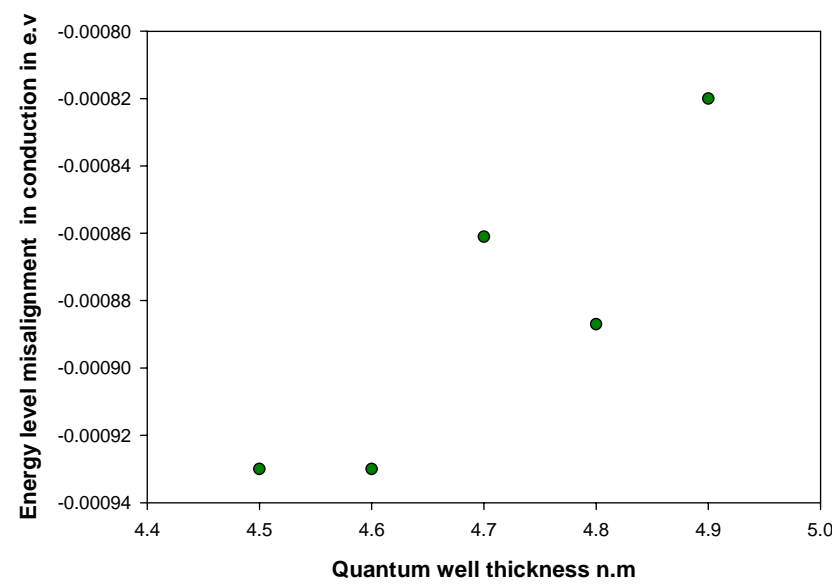

Figure 3.11: Alignment of discrete energy levels in the conduction band for InN bandgap of 1.9e.v and different combination of quantum well thickness 
From the plots Fig 3.10 we see that the misalignment between the energy levels of the quantum wells in the active region increases as one approach the quantum wells close to the p-side. This misalignment of carriers reduces the tunneling of carriers across the barriers offsetting the inhomogeneous carrier concentration across the quantum wells. This effect may increase the uniformity distribution of carriers across the quantum wells.

The overall effect of this design is to improve uniformity of distribution of carriers across the active region, which may well reduce the threshold current density of the laser diode. Based on the above predictions, if uniformity is achieved the AlGaN blocking layer responsible for preventing the carriers from overflowing can be removed. The problem with $\mathrm{AlGaN}$ blocking layer is that it increases the prevents the overflow of carriers(electrons) at the cost of increased series resistance and also presents a barrier to the free flow of holes into the quantum well from the p-region.

\subsubsection{Double barrier quantum well:}

The literature says that for operation at higher wavelengths the carriers at higher energy levels must settle down to lower energy states. But the scattering length of the carriers may well be larger than the thickness of the quantum wells. This will cause loss of the carriers at higher energy levels and an increase in threshold current density.

The threshold current density may decrease if

- The carriers at higher energy levels travel sufficient distances across the quantum well to scatter to lower energy levels.

- The carriers at higher energy levels tunnel across the barriers easily and carriers at lower energy levels have further reduced tunneling than conventional quantum wells.

Scattering of carriers in a quantum well from higher energy levels to lower energy levels is through electron-phonon interaction. Literature suggests [Holyank, 1980] that quantum well structures encourage stimulated electron-phonon interaction and which may well lead to laser actions well below the lowest energy level of the quantum well. Based on the above the following structure was proposed. 

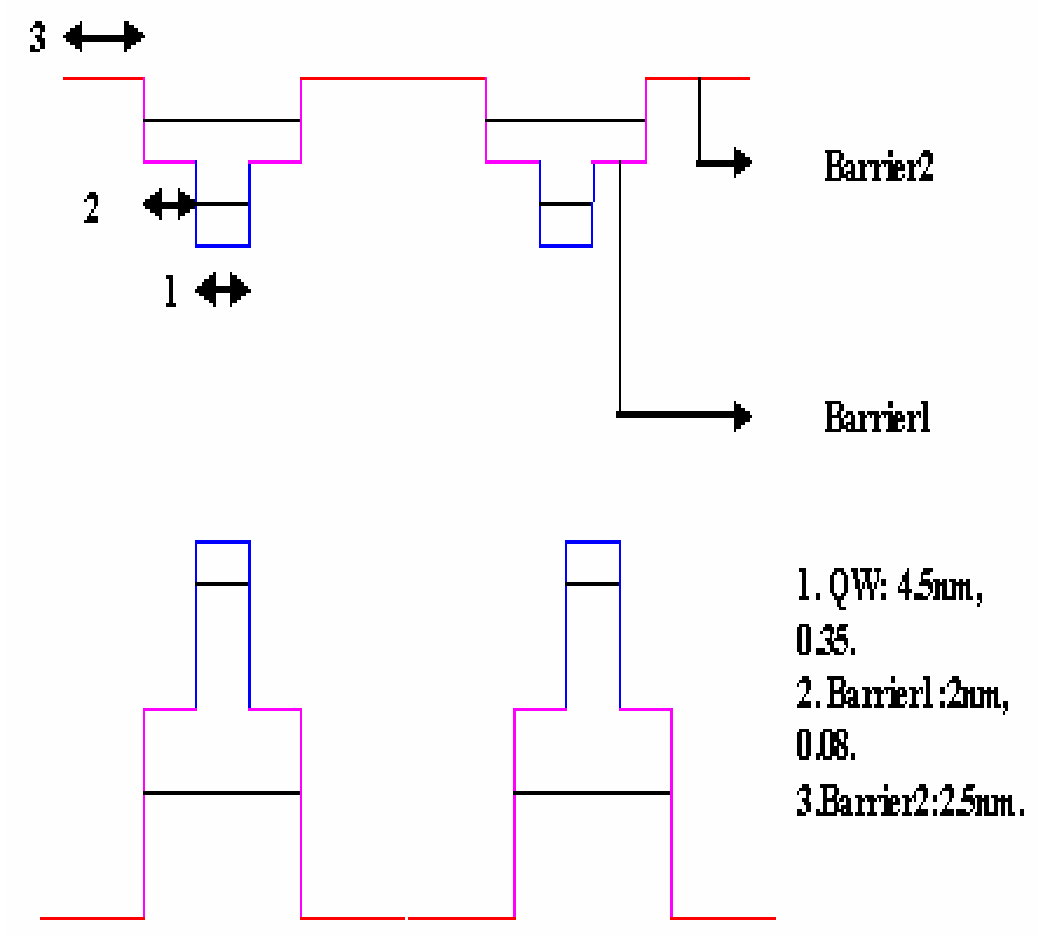

Figure 3.12 Appendix B: Simple double barrier for emission wavelength of 450nm and for InN bandgap of 1.9e.v.

\section{Working of the double barrier quantum well:}

A wider quantum well was created above a narrow quantum well Fig 3.12. The narrow quantum wells determine the lowest energy states available and are similar to the conventional structures. The wider quantum well is expected to collect more carriers than the conventional as it has higher density of states and is wider. The wider quantum well enhances the thermalization of carriers (electrons). The increased density of states leads to enhanced electron-phonon interaction leading to stimulated electron phonon interaction. At the same time the carriers at higher energy levels easily tunnel across the barrier when compared to carriers at lower energy states. The tunneling increases further thermalization of carriers to lower energy states. This reduces the carrier overflow. The net effect is a reduction in the threshold current density of the laser diode. 
Analysis of the lowest energy state in the wider quantum well gives an idea about the current overflow in the quantum well. The deeper the lowest energy state of the wider quantum well is the lower is the current overflow.

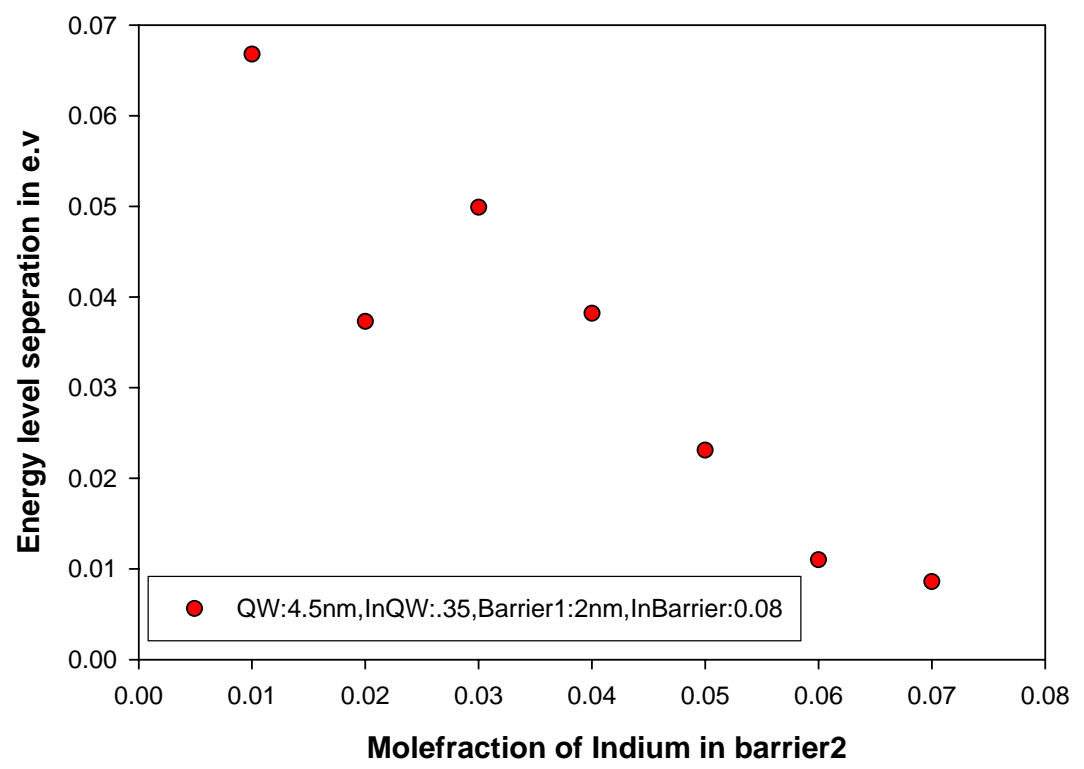

Figure 3.13 Appendix B: Lowest energy state separation from the edge of barrier2 for emission wavelength of $450 \mathrm{~nm}$

But this is not the only criteria deciding the overflow. A high conduction band offset is desired so that carriers scatter deep into the quantum well.

A plot Fig 3.13 of the lowest energy state as function of indium mole fraction in the second barrier suggests that with decreasing indium mole fraction or potential well depth of the wider quantum well, the lowest energy state energy of the wider quantum well decreases, in general. The trend breaks at an indium mole fraction of 0.02 . The mole fraction of indium at which the trend breaks may vary with varying indium mole fraction 
of the first barrier. This decrease in energy of lowest energy state is advantageous as the carriers are confined deep in the potential well and are thus prevented from overflowing or escaping the well.

These designs may prove to be effective provided that the conduction band offset is not too low as is the case for InGaN UV laser diodes. Literature has indicated that the low offset increases the carrier overflow at high current densities required for laser diode [Nagahama, 2002]. This overflow requires compensation in the form of increased carrier density and thereby increasing the threshold current density.

\subsubsection{Novel structure for cladding layer 1D Photonic Crystal (PC):}

A 1D - photonic crystal is composed of alternating stacks of layers having a low and high index of refraction Fig 3.14. These structures reflect light at any polarizations, any incidence angle and over a wide range of wavelengths.

\subsubsection{Effective index of a 1D PC:}

A wave propagating along the direction of periodic variation experiences an effective index and it has been shown that this effective index is less than 1 [Weizong, 2002].

The formula for wave vector $\mathrm{k}$ along the direction of periodic variation is given by:

$$
k=\frac{1}{p} \arccos \left(\cos \left(v_{1} k_{0} h_{1}\right) \cos \left(v_{2} k_{0} h_{2}\right)-\frac{1}{2}\left(\frac{v_{1} \eta_{2}}{v_{1} \eta_{1}}\right) \sin \left(v_{1} k_{o} h_{1}\right) \sin \left(v_{2} k_{o} h_{2}\right)\right.
$$

[Abdulhalim, 2000]

The real part of the wavevector divided by the wavevector in free space gives the effective refractive index of the wave along the periodic variation. 


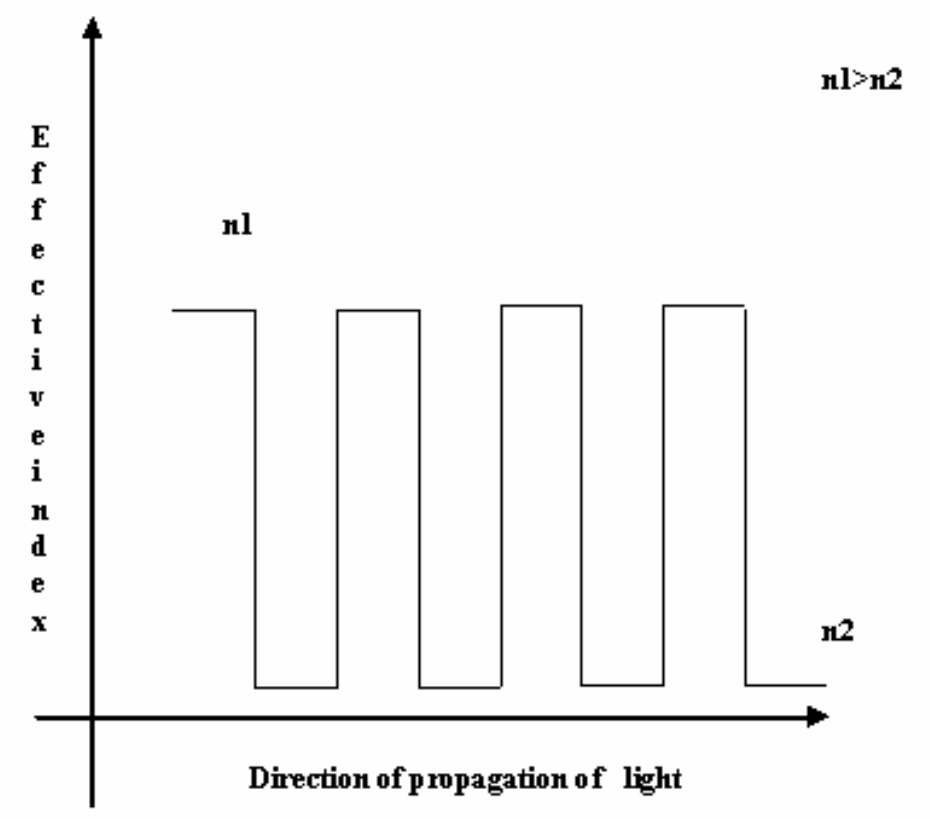

Figure 3.14: Variation of refractive index of a 1D photonic crystal in direction of propagation of light

\section{Effective index vs Angle of incidence}

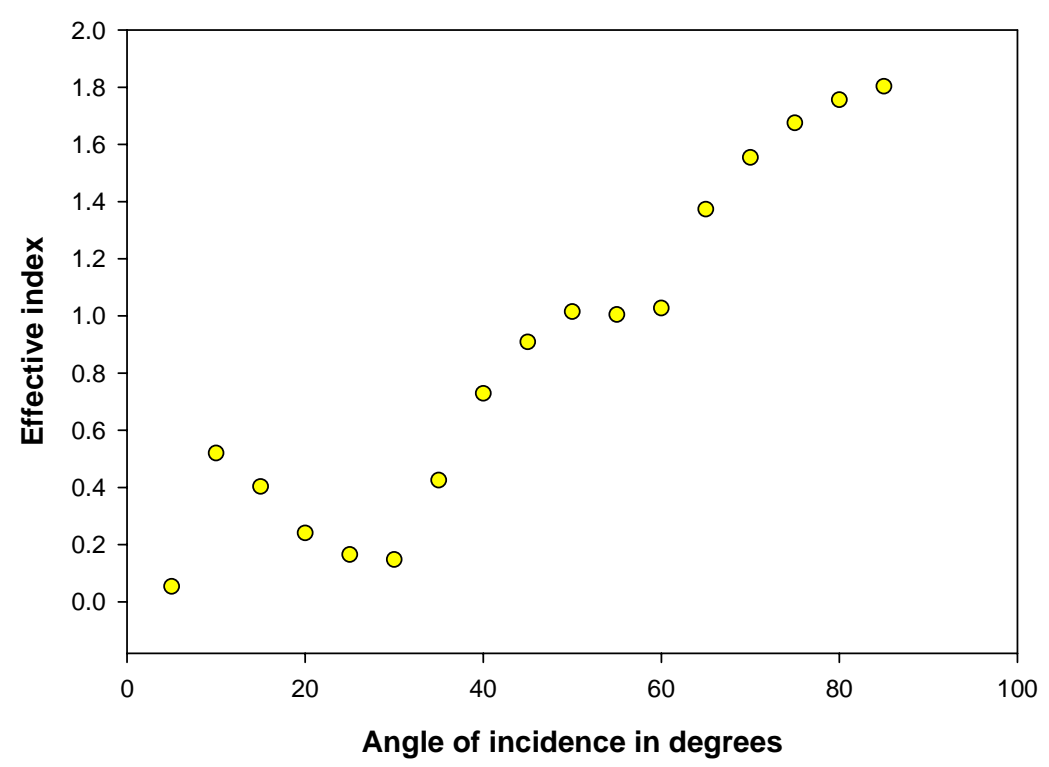

Figure 3.15: Effective index of 1D photonic crystal $\mathrm{Al}_{0.32} \mathrm{Ga}_{0.68} \mathrm{~N} /$ air for different incidence angles, when incident from $\mathrm{GaN}$ 


\subsubsection{Problems with AlGaN:}

As stated before the mole fraction of AlGaN cannot be increased beyond 0.32 further increase in Aluminum mole fraction reduces the carrier concentration in the AlGaN. This limits the value to which the refractive index of $\mathrm{AlGaN}$ can be decreased.

\subsubsection{Photonic crystal as cladding:}

A photonic crystal as a cladding can serve as a better cladding layer than AlGaN due to its low effective refractive index. This serves two purposes it confines the electric field better than the conventional $\mathrm{AlGaN}$ cladding layer thereby reduces the thickness of the GaN optical guiding region which reduces the defect density. The contacts can be deposited directly on the optical guiding GaN layer. 


\section{ETCHING TECHNIQUES FOR GaN}

4.1. Overview: This chapter describes various etching techniques for $\mathrm{GaN}$ and the preferred etching technique.

4.2. Introduction: The fabrication of a laser diode starts with the growth of the layers on a sapphire substrate. For fabricating a laser diode from these layers need to be etched down to the $\mathrm{n}-\mathrm{GaN}$ to deposit contacts. Etching also creates the resonant cavity and side walls. For the side walls to act as mirrors, etching must produce smooth and vertical sidewalls. The sapphire substrate is large enough usually around 2.5 inches to fabricate multiple laser diodes on a single wafer.

Etching is typically done by masking the device and performing wet or dry etching. Control of etch depth is necessary for proper operation of the device. Improper control of the etch rates or the etching process can lead to undercut or rough sidewalls and deposition of contacts at depths at which the device performance is severely degraded.

\subsection{Types of etching:}

\subsubsection{Wet etching:}

High quality GaN substrates are highly resistant to chemical etching. Wet etching is basically crystallographic etching. The etch rates are different depending on the atomic plane being etched [Stocker, 1998]. Undercut is another problem with wet etching which makes wet etching undesirable for GaN. 


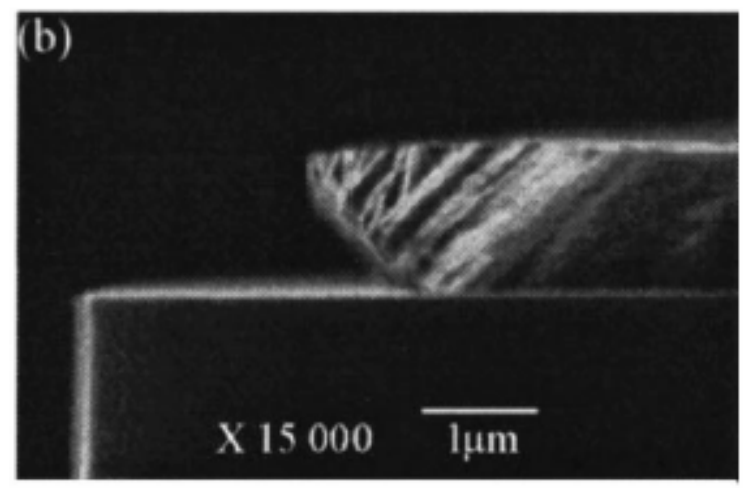

Figure 4.1 [Stocker, 1998]: Undercut in GaN resulting from wet etching

\subsubsection{Dry Etching or plasma etching:}

Dry etching does not use solutions to remove material from the surface of the wafer. Instead the process uses gases. The plasma generated from the gas mixture generates volatile byproducts which are drained though a pump .In some cases nonvolatile products are generated along with volatile products these are removed through process of sputtering or physical removal.

The etching process occurs through three mechanisms:

1. Through chemical reactions that consume the material, using chemically reactive gases or plasma.

2. Physical removal of the material, usually by momentum transfer to the wafer by ions generated.

3. A combination of both physical removal and chemical reactions.

Like wet etching, dry etching also follows the resist mask patterns on the wafer, i.e., it only etches away materials that are not covered by mask material (and are therefore exposed to its etching species), while leaving areas covered by the masks almost (but not perfectly) intact. 
Selectivity is the most important criteria in the removal of species. The species selected must be able to attack exposed material readily while leaving out the mask intact. Usually the mask is also attacked. For this reason a very high selectivity is needed between material being etched and mask covering it.

\subsubsection{Chemical etching:}

Plasma etching is a purely chemical etching in which free radicals control the etch rates rather than the ions generated. The free radicals generated in the plasma diffuse to the wafer surface being etched. The radicals are adsorbed on the wafer surface where they react with the material to be etched. It necessary that the chemical reaction leads to volatile by products so that fresh layer of the material is exposed. If the by products formed are not volatile they stay on the surface and the material may not be etched no matter for any time length. Chemical etching can lead to undercutting or isotropic etching due to the diffusion of free radicals.

\subsubsection{Physical etching:}

If anisotropy is of main concern then bombarding the surface with ions is a good option. The ions due to effect of electric field are confined in a particular direction and hence do not diffuse. The energetic ions dislodge atoms from the material surface from the impact of the ions on the surface. In this process the momentum of the ions is used up in breaking up the bonds on the surface. This process is non selective as the ions tend to etch the mask at the same rate as the material.

\subsubsection{Reactive Ion Etching:}

This process combines both the physical and chemical processes. Both ions and free radicals are generated. The ions are accelerated towards the wafer by the negative DC bias created on the wafer and the free radicals diffuse to the surface. A combination of these two processes helps to increase the etch rate. Also any non volatile by products are expelled from the surface through sputtering action exposing a fresh layer of material to free radicals and ions. 


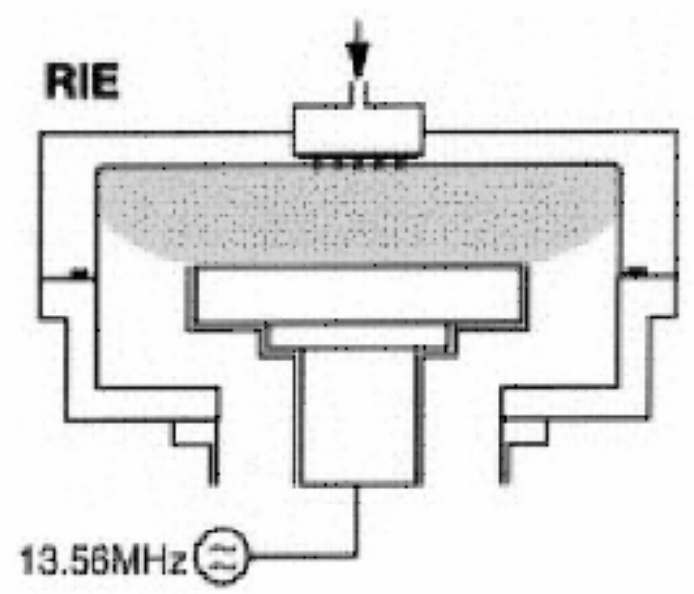

Figure 4.2 Oxford Plasma Systems: RIE System

\subsubsection{ICP -RIE Systems:}

The drawback with RIE system is that order to increase the density of the ions and free radicals for higher etch rates the a large power is applied at the wafer This serves the purpose of high ion density but at the same time increase the energy of the ions bombarding the surface of the wafer due to higher DC bias. This high energy bombardment can lead to surface damage and deterioration of the optical and electrical properties of the material.

ICP (Inductively Coupled Plasma) is a tool where the density of the plasma can be increased at lower DC biases. This is done by using two RF sources which independently control the density of the plasma and the energy of the ions bombarding the surface. 


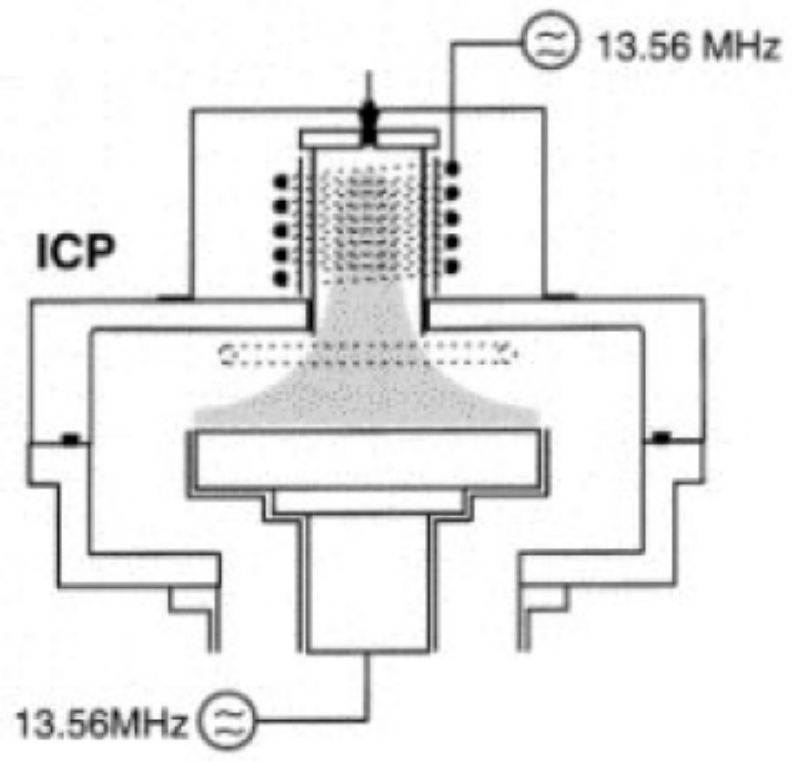

Figure 4.3 Oxford Plasma Systems: ICP-RIE

Improved surface characteristics and higher etch rates for $\mathrm{GaN}$ were realized using this ICP set up [Ryan, 1999].

\subsubsection{Types of plasmas used for etching GaN:}

Chlorine based ICP-RIE systems have been used for commercially producing laser diodes. Chlorine gases when mixed with other gases give controllable; high etch rates and vertical side walls at low DC biases with reduced surface damage. Some of the systems used are $\mathrm{Cl}_{2} / \mathrm{Ar}, \mathrm{Cl}_{2} / \mathrm{SF}_{6}, \mathrm{Cl} / \mathrm{N}_{2}, \mathrm{Cl}_{2} / \mathrm{Ar} / \mathrm{H}_{2}, \mathrm{BCl}_{3} / \mathrm{Cl}_{2}, \mathrm{H}_{2} / \mathrm{Cl}_{2}$. But chlorine based systems are corrosive and hazardous. For this reason research has been focused towards fluoride based etching. Etching $\mathrm{GaN}_{\text {in }} \mathrm{SF}_{6}$ plasma is currently under research.

\section{Fluorides:}

Fluorides have not been considered as they form non volatile products with gallium like $\mathrm{GaF}_{3}$ on the wafers [Basak, 1997]. This limits the etch rate of $\mathrm{GaN}$ in $\mathrm{SF}_{6}$ plasma. As the debris has to be removed with sputtering from additional ions. This limitation of etch rates 
can be removed provided the non volatile products are sputtered out from the wafer surface. It is this point which continuous to drive research in fluoride based etching of GaN. The etch rate of GaN also depends on the growth conditions [Basak, 1997]. Experimental results have been reported for $\mathrm{GaN}$ with vertical side walls and etch rates of $29 \mathrm{~nm} / \mathrm{min}$ [Cheung, 1999] using RIE systems. Inclusion of Ar along with fluorides has been suggested for increasing the etch rates. The heavy Ar ions can contribute significantly to the etch rates in the form of sputtering. Other fluoride based systems under consideration are $\mathrm{CF}_{4}, \mathrm{CHF}_{3}$ which included $\mathrm{Ar}$ gas as an additional species to increase the etch rate of $\mathrm{GaN}$ [Heon, 1995]. 


\section{EXPERIMENT-ETCH RATE OF GaN}

5.1. Overview: This chapter discusses about the experimental procedure for determining the etch rates of $\mathrm{GaN}$ in $\mathrm{SF}_{6}$ plasma and issues related to the vertical side wall.

\subsection{Procedure for determining etch rates:}

For determining the etch rates of $\mathrm{GaN}$, sections of the GaN wafer are masked Fig 5.1 and the sample is etched in the ICP-RIE system under varying conditions of chamber pressure, flow rates of the gases and ICP powers. The mask selected should have high selectivity with respect to the material being etched. In our experiment the material being etched is GaN. In other words the mask should not be etched completely during the process.

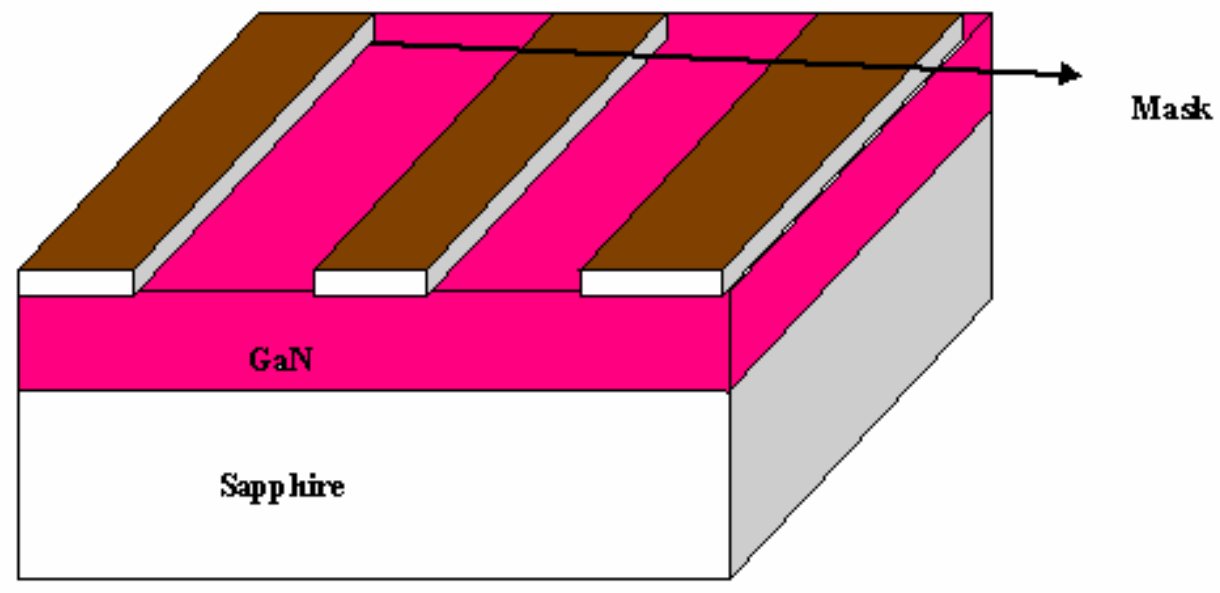

Figure 5.1: Unetched Sample 
After processing Fig 5.2 the sample in the ICP-RIE system the mask has to be removed to measure the depth between adjacent etched and unetched surfaces of the GaN sample Fig 5.3 .

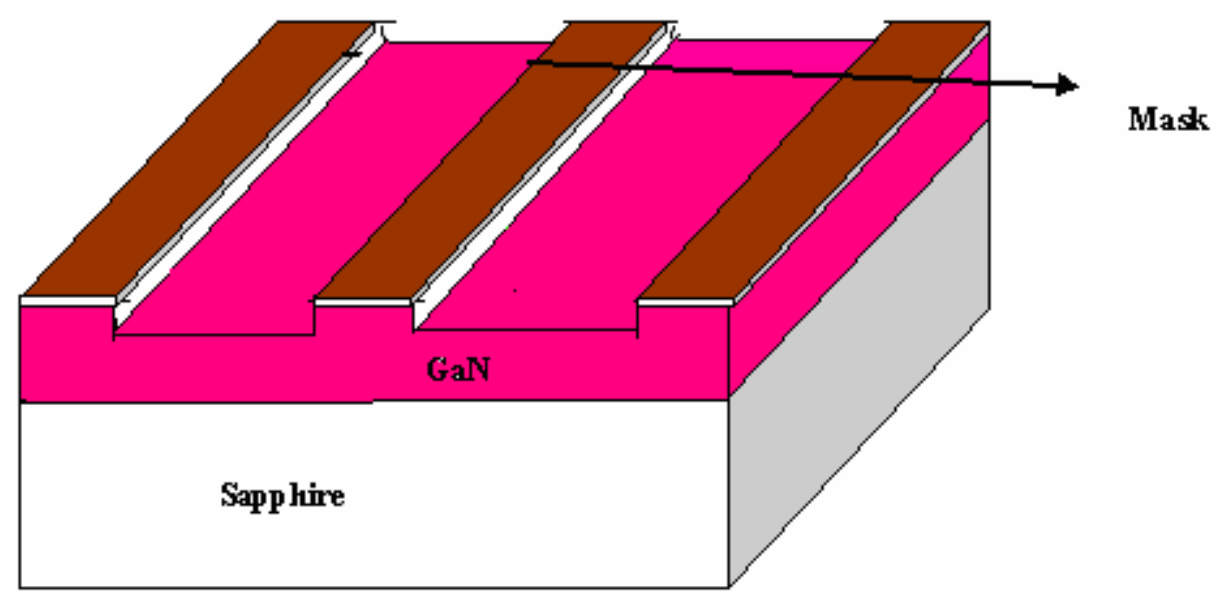

Figure 5.2: Etched Sample

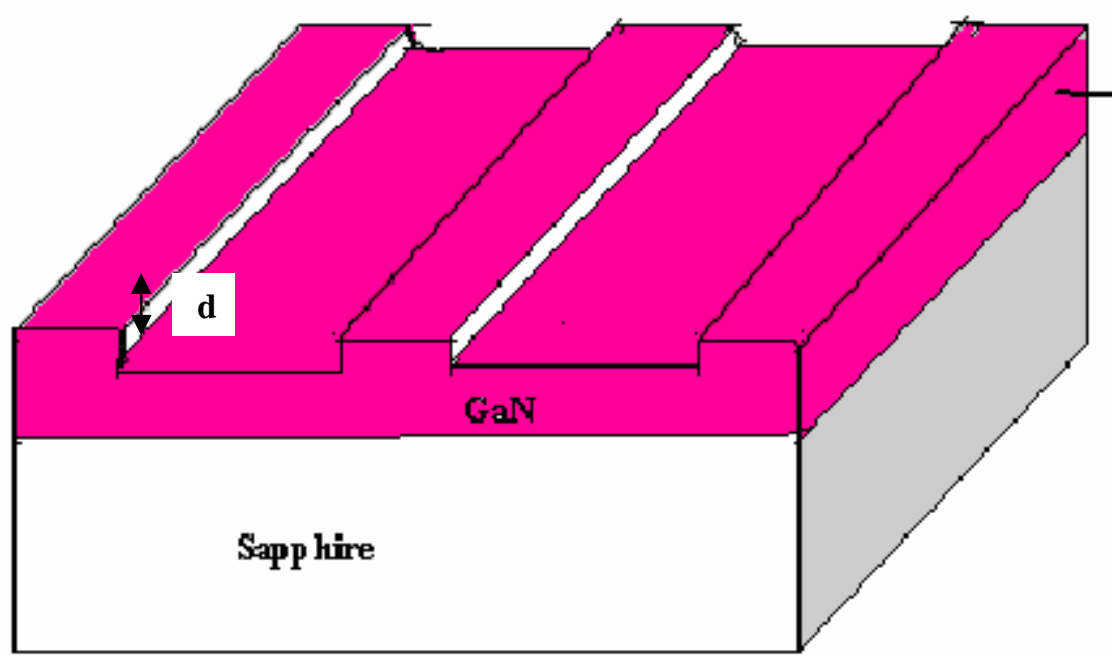

Figure 5.3: Sample after mask removal 
The etch rate is now given by $\boldsymbol{d} / \boldsymbol{t}$.

$\mathrm{d}$ : The depth between the etched and unetched surfaces.

t: The etch process time.

\subsection{Experimental procedure:}

\subsubsection{Gas Species used for etching:}

Sulphur hexafluoride $\mathrm{SF}_{6}$ was used for creating plasma in the ICP-RIE system.

\subsubsection{Types of Masks:}

\subsubsection{Silicon-di -oxide (SiO2):}

\section{Etching $\mathrm{SiO}_{2}$ and $\mathrm{SiO}_{2}$ stripes}

Initially $\mathrm{SiO}_{2}$ was considered as a mask for the $\mathrm{GaN}$ samples. For determining the etch rates of $\mathrm{SiO}_{2}$ in $\mathrm{SF}_{6}$ plasma, a $1 \mu \mathrm{m}$ thick $\mathrm{SiO}_{2}$ was grown on a silicon wafer using PECVD system. The sample was then masked with pattern of positive photo resist (AZ5214).The stripe pattern was obtained by using a laser mask during exposure of the photo resist. The photo resist was spun at $2000 \mathrm{rpm}$ to obtain a thickness of $3 \mu \mathrm{m}$.

\section{Etch rates of $\mathrm{SiO}_{2}$ :}

The sample was then etched in the ICP-RIE system under varying parameters. The process time was $2 \mathrm{~min}$. The sample was then removed from chamber and the remaining photo resist was stripped off by soaking the sample in acetone. Ultrasonic bath was performed to remove any hardened photo resist.

The etched depth of silicon dioxide was measure using the alpha-step (profilometer). From the measured etch rates Fig 5.4 and Fig 5.5, we observe that the etch rates of $\mathrm{SiO}_{2}$ are at minimum $100 \mathrm{~nm} / \mathrm{min}$. The maximum thickness of $\mathrm{SiO}_{2}$ that could be grown in the PECVD was only $1 \mu \mathrm{m}$. A thicker growth deteriorated the material quality of $\mathrm{SiO}_{2}$ which could have led to unpredictable etch rates of $\mathrm{SiO}_{2}$. 


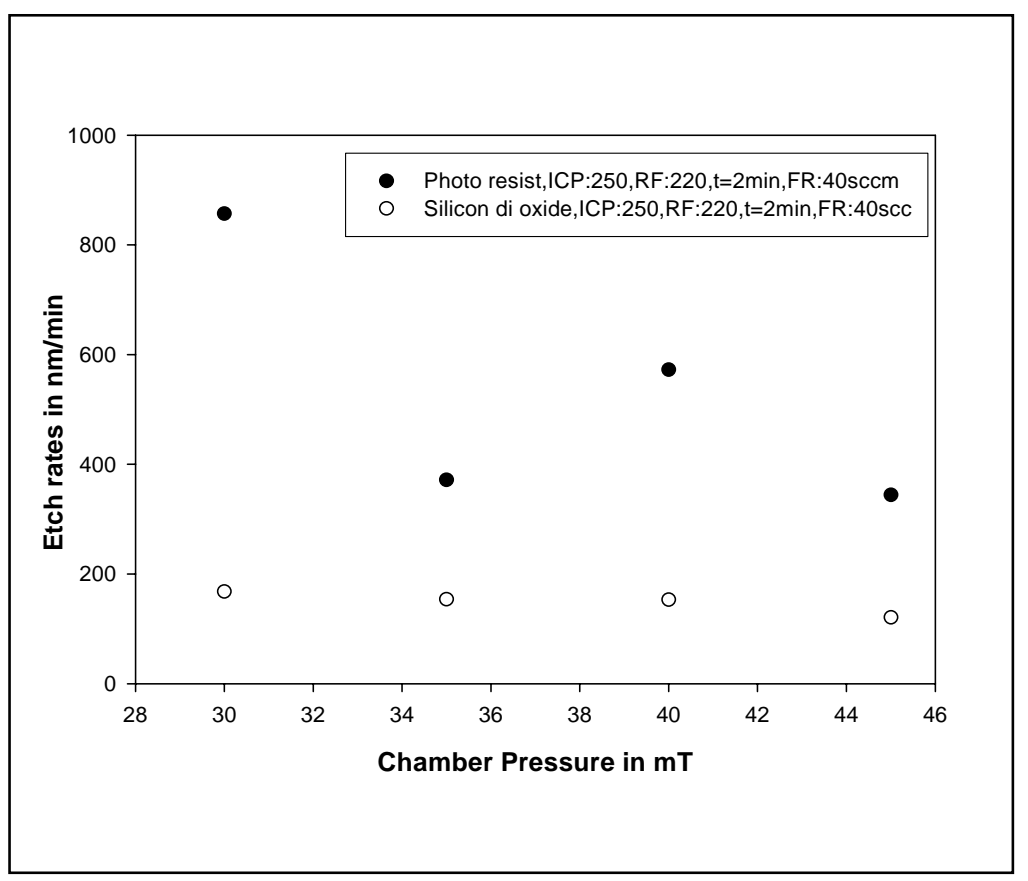

Figure 5.4: Etch rates of Silicon-dioxide and Photoresist vs. Chamber Pressure

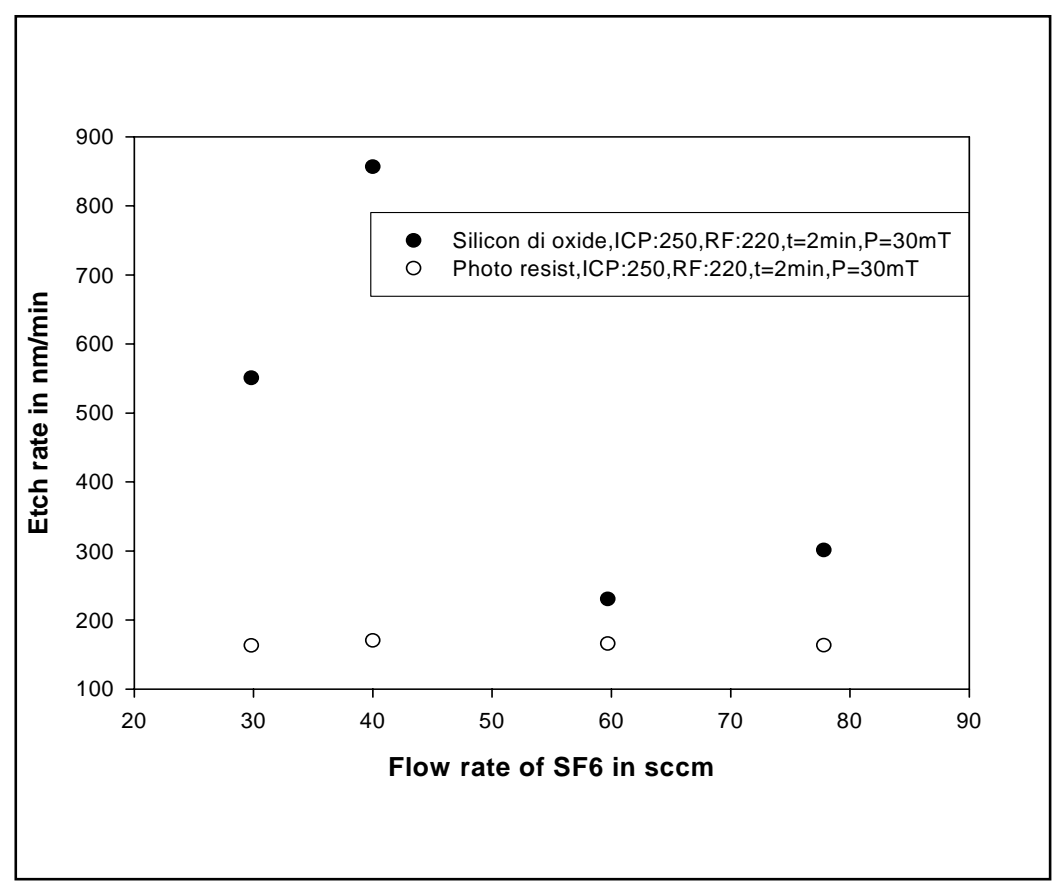

Figure 5.5 Etch rates of Silicon-dioxide and Photoresist vs. Flow rate of $\mathrm{SF}_{6}$

Based on the above the maximum time for which a process could be executed was around $10 \mathrm{~min}$. The GaN sample was etched with $\mathrm{SiO} 2$ as a mask for a process time of $10 \mathrm{~min}$. 
The etching could not be confirmed when viewed under the SEM. We suspected that the etch rates of $\mathrm{GaN}$ were too low and that the side wall profile was highly isotropic. Based on the above results we decided that $\mathrm{GaN}$ had to be etched for a longer time to achieve greater depths to actually view the side wall. Literature also states that the etch rates of $\mathrm{GaN}$ in $\mathrm{SF}_{6}$ plasma are very low $50 \mathrm{~A} / \mathrm{min}-290 \mathrm{~A} / \mathrm{min}$ and side walls tend to be isotropic for fluorides plasmas[Basak, 1997], [Cheung, 1999] the sample had to be etched for longer time and $\mathrm{SiO} 2$ was not a good choice as a mask

\subsubsection{Chrome mask:}

Metallic chrome has an etch rate of $1 \mathrm{~nm} / \mathrm{min}$ in $\mathrm{SF}_{6}$ plasma [Williams,1996] and with such a high selectivity chrome was obvious choice as a mask. Stripes of chrome of thickness $160 \mathrm{~nm}$ were patterned on the GaN sample through the process of photolithography using positive photo resist (AZ5214), sputtering and liftoff. The samples were etched for an hour in the ICP-RIE system under varying parameters. The remaining chrome was etched off using the chrome etchant. The depths were measured using a profilometer. In order to get maximum the etch rate, as a starting point the system was operated under maximum conditions $\mathrm{ICP}=250 \mathrm{~W}, \mathrm{RF}=220 \mathrm{~W}, \mathrm{FR}=60 \mathrm{sccm}$ for varying chamber pressures Fig 5.6

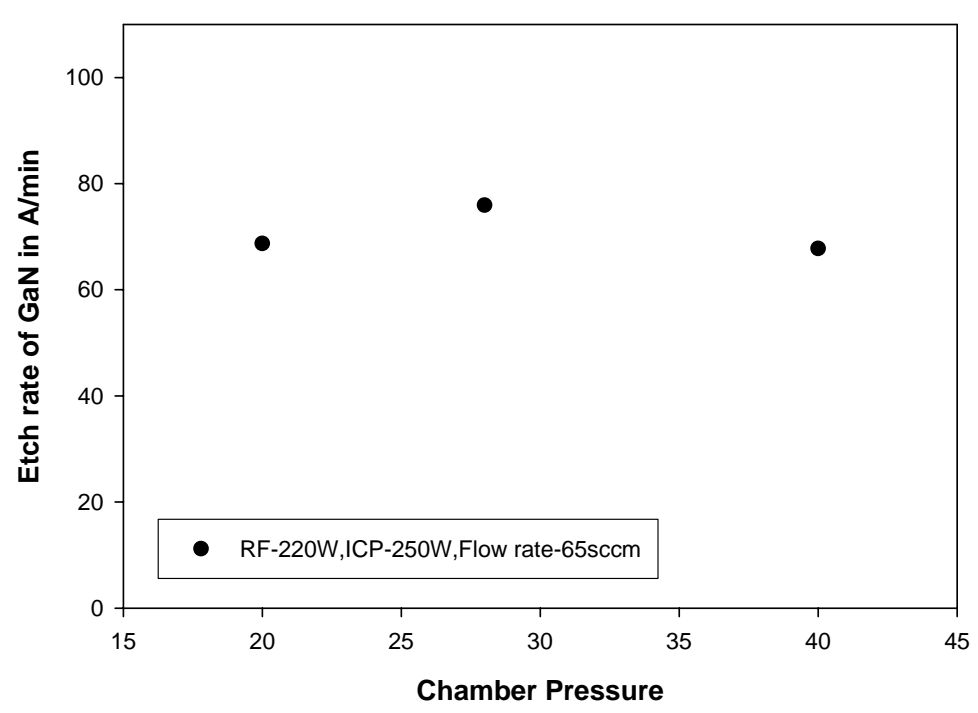

Figure 5.6: Etch rate of GaN vs Chamber pressure 
From Fig 5.6 we see that the etch rate increase with an increase in the chamber. The etch rate drops off at higher pressure. At higher pressures the electrons loose energy due to scattering effects and do not have enough energy to dislodge electrons from other atoms to create free radicals. The density of plasma is therefore reduced at higher pressure. This results in a drop in the etch rates. The decrease in the plasma density shows up in the form of a variation in the DC bias from $300 \mathrm{~V}-350 \mathrm{~V}-325 \mathrm{~V}$. This is a significant result, the DC bias determines the energy of the positive ions striking the sample and at low pressures contribute significantly to the etch rates.

Effect of flow rate on the etch rate was inspected. The flow rate may determine the time for which the ions and radicals remain in the chamber. Taking the maximum value of etch rate into account the flow rate was varied Fig 5.7.

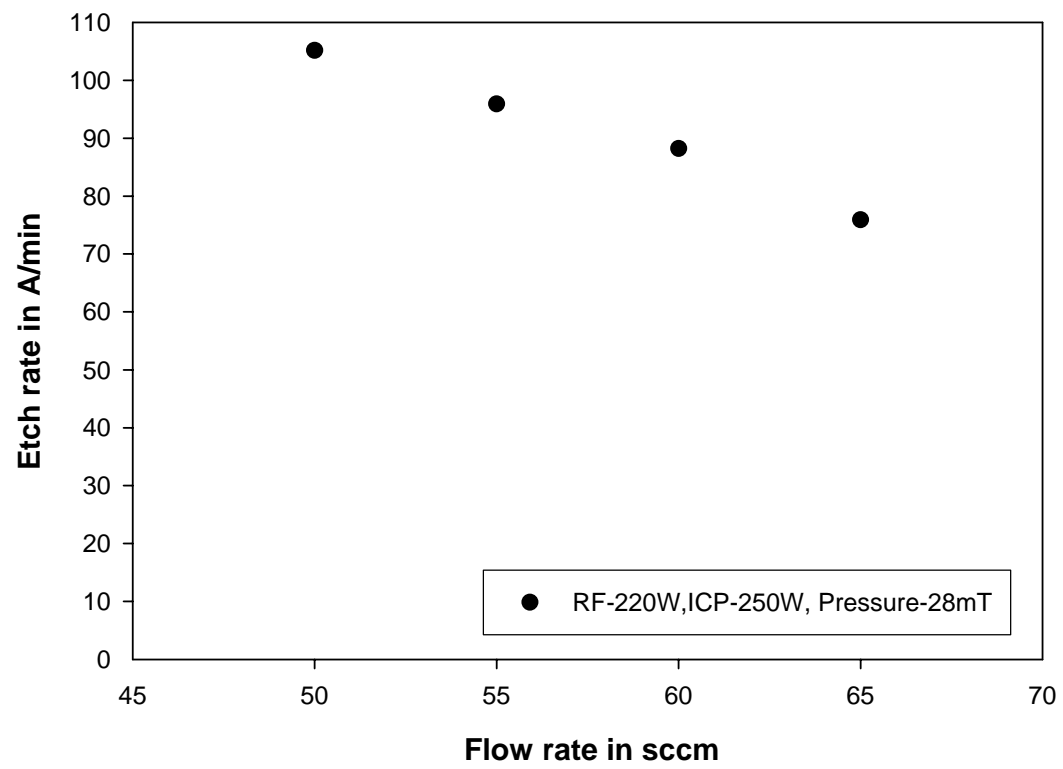

Figure 5.7 : Etch rate of $\mathrm{GaN}$ vs Flow rate of $\mathrm{SF}_{6}$

From the plot Fig 5.7 we see that the etch rate drops off at higher flow rates. With an increase in the flow rate the throttle valve opens more. This may be the critical factor determining the etch rate. When the position of the throttle is wide open at high flow 
rates it is difficult to maintain plasma under such conditions. This may result in a decrease in the density of plasma or the duration for which the radicals and ions stay in the chamber. These factor directly effect the etch rates and may be responsible for the decrease in the etch rates.

But the etch rates obtained are not optimized. Identical or better etch rates could be obtained at lower pressure as sputtering of ions plays a major role in determining the at low pressure, due to less scattering effects in the chamber Fig 5.8.

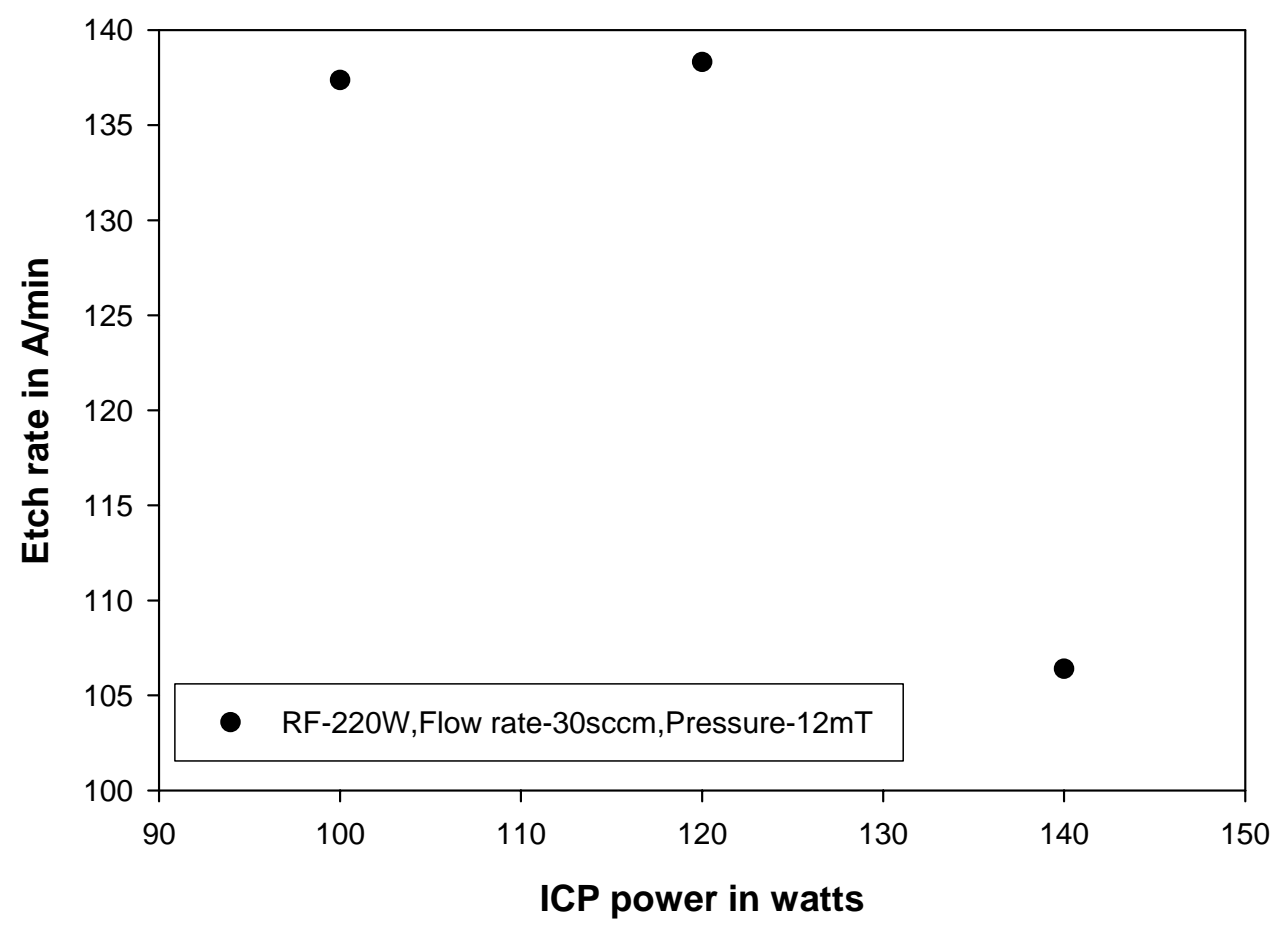

Figure 5.8: Etch rate of GaN vs ICP power

From the plots Fig 5.8 we see that higher etch rates were obtained at lower pressures and lower ICP powers, when compared to the process at high pressure and high ICP powers. This indicates that sputtering by ions has played a major role in determining the etch rates. The etch rate drops off at a higher ICP power this is due to lower density of gas species trying to absorb a high ICP power, as a result of this the systems tries to stabilize 
the plasma by increasing the density of gas species and at same time trying to maintain the pressure. This gives plasma that is unstable with time. Hence lower etch rates.

The etching of GaN by $\mathrm{SF}_{6}$ plasma was independently confirmed by the following result from the SEM .

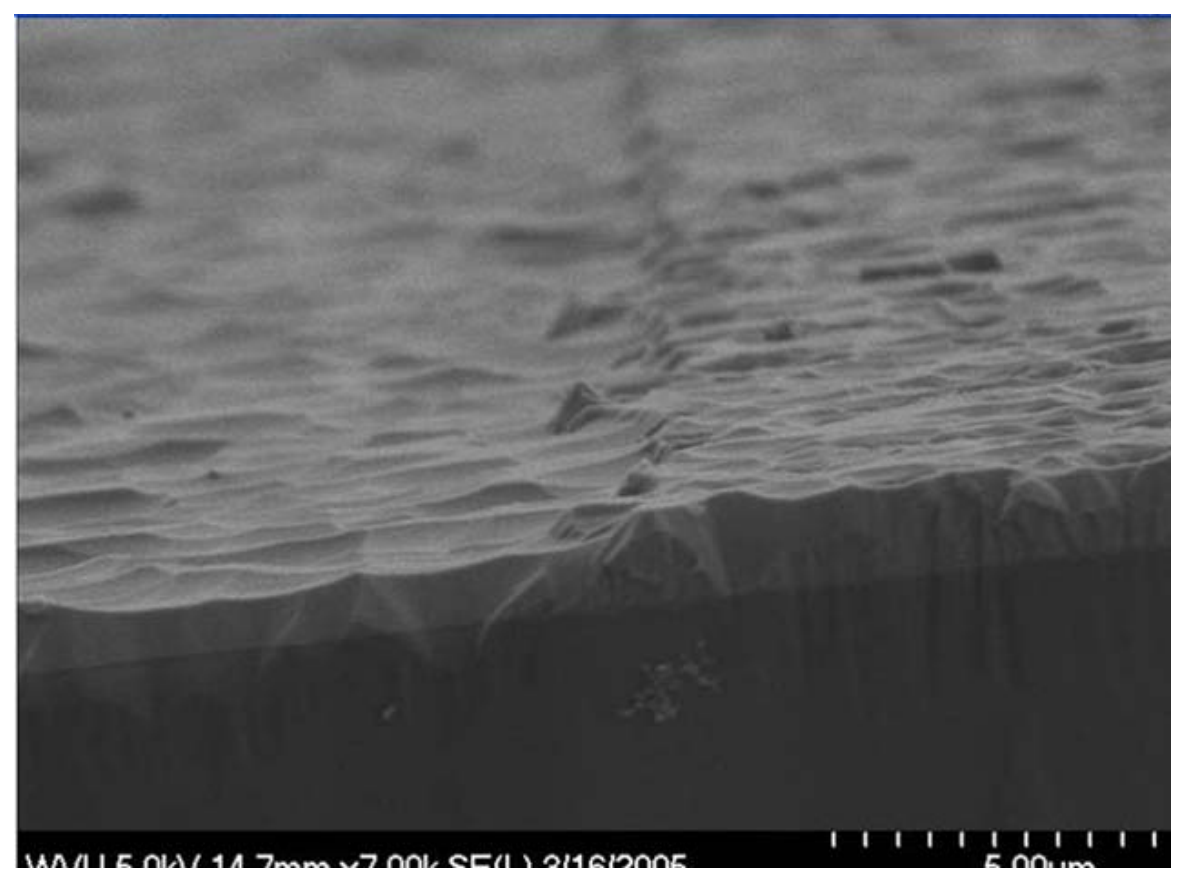

Figure 5.9: SEM image of $\mathrm{GaN}$ at ICP=170W, RF 150W, FR=30sccm, $\mathrm{P}=17 \mathrm{mT}$

The maximum DC bias from the processes was 360V. The SEM image shows a side wall that is not well defined.

Well defined and vertical side walls were reported for $\mathrm{GaN}$ samples etched in $\mathrm{SF}_{6}$ with RIE systems Fig 5.10. The DC bias reported was at 440 at a pressure of $15 \mathrm{mT}$ and $40 \mathrm{sccm}$. The sample was etched for $17 \mathrm{~min}$. 


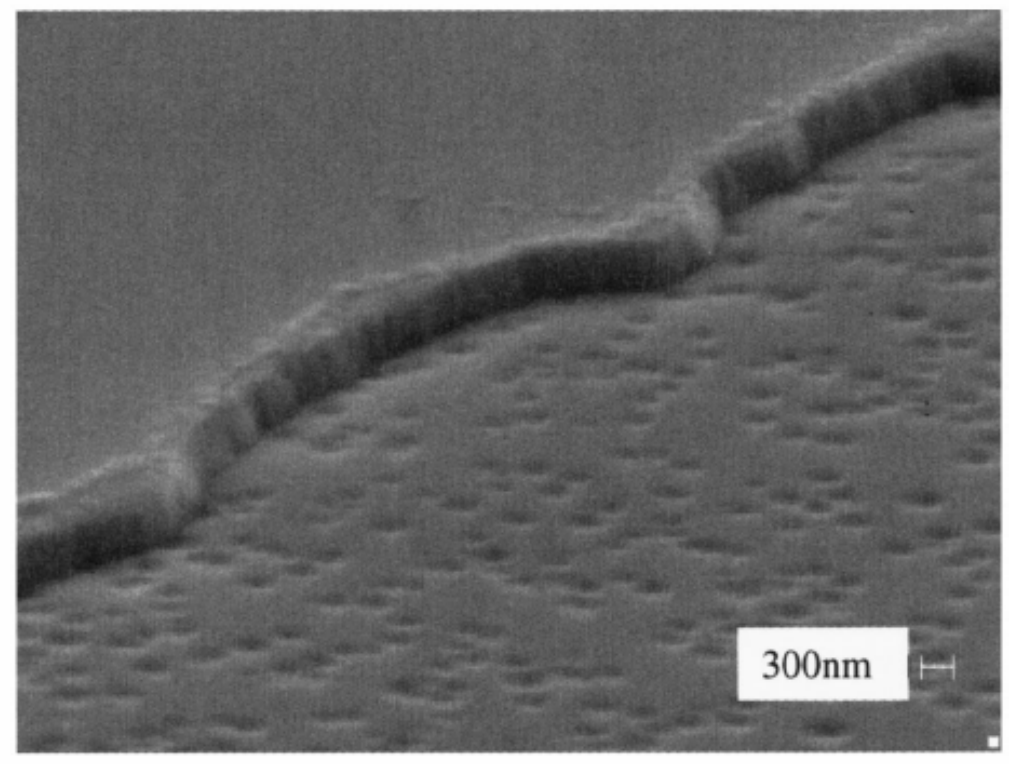

Figure 5.10 [Cheung, 1999]: sample was etched for 17min

The literature results also supports our assumptions that at high DC biases and low pressures the etching is dominated by sputtering action.

Another observation from the literatures is that etching dominated by sputtering is fast and anisotropic in nature. 


\section{CONCLUSIONS AND FUTURE WORK}

Designs for the active region have been proposed to achieve uniform distribution of carriers across the active region of the quantum well. To achieve lasing at low energies at a threshold current density compared to the conventional laser diode structures. These designs can also be applied to InGaN LEDs to prevent the overflow of carriers in these

structures. Applying these results towards research and development of AllnGaN deep UV laser diodes. A 1D photonic crystal was investigated as a possible replacement for the $\mathrm{AlGaN}$ cladding region.

Etch rates of an undoped GaN grown in the MOCVD were determined by etching the sample in the ICP-RIE system using $\mathrm{SF}_{6}$ as gas species. Vertical side walls could not be observed due to the limitations of the DC bias. The process is sufficient for fabricating an LED as vertical side walls are not of concern in LEDs. But the system parameters are not stable for long processes. In order to use these results-etch rates crystals of the same quality have to be produced.

As for the future work InGaN laser diodes based on the proposed structure have to be grown and tested. The parameters like quantum well thickness, barrier thickness have to be varied to get the lowest threshold current density for the laser diode that is the optimum laser diode design. The ICP-RIE process needs to be optimized to get higher etch rates and vertical side walls. 


\section{REFERENCES}

[Abdulhalim, 2000] I Abdulhalim J. Opt. A: Pure Appl. Opt. 2 (2000) L9-L11.

[Akita, 2004] Katsushi Akita, Takao Nakamura, Hideki Hirayama.

phys. stat. sol. (a) 201, No. 12, 2624-2627 (2004) / DOI 10.1002/pssa.200404980

[Alivova, 2003] Ya. I. Alivova ,E. V. Kalinina and A. E. Cherenkov, D. C. Look,B. M. Ataev and A. K. Omaev, M. V. Chukichev, D. M. Bagnall, Appl .Phys. Lett,Vol 83, No 238 December 2003.

[Bahaa, 1999] Bahaa E. A. Saleh, Malvin carl teich , Fundamentals of Photonics, John Wiley and Sons,Inc 1991.

[Basak , 1997] D Basaky, M Verduz, M T Montojoz, M A Sanchez-Garciay,F J Sanchezy, E Mũnozy and E Callejay, Semicond. Sci. Technol. 12 (1997) 1654-1657.

[Bour, 2000] D. P. Bour, M. Kneissl, C. G. Van de Walle, G. A. Evans, L. T. Romano, J. Northrup, M. Teepe, R. Wood, T. Schmidt, S. Schoffberger, and N. M. Johnson, IEEE J.Quantum.Elec, Vol. 36, No. 2, February 2000.

[Bradley, 2003] S. T. Bradley, S. H. Goss, L. J. Brillson, J. Hwang and W. J. Schaff, American Vacuum Society. @DOI: 10.1116/1.1627331\#, 2003.

[Cheung, 1999] R. Cheung, S. Withanage, R. J. Reeves, S. A. Brown, and I. Ben-Yaacov, C. Kirchner and M. Kamp,Appl.Phys.Lett,Vol 74,No21,24 May 1999.

[Chichibu, 1999] S.Chichibu, T. Sota, K. Wada, S. P. DenBaars, S. Nakamura, MRS Internet J. Nitride Semicond. Res. 4S1, G2.7 (1999). 
[Coldren, 2995] Larry A Coldren, Scott W.Corzine, Diode Lasers and Photonic Integrated Circuits, Wiley and Sons,Inc 1995.

[Czyszanowski, 2001] T Czyszanowski1 and W Nakwaski , Inst.Phys Publishing,J.Phys,D: Appl.Phys,J.Phys.D: Appl. Phys. 34 (2001) 2346-2352 PII: S0022$3727(01) 24451-9$.

[Ferhat, 2002]M.Ferhat,J.Furthmuller, F.Bechstedt, Appl.Phys.Lett, Vol 80 , No 8 ,February 2002.

[Hansen, 1999] M. Hansen, A.C. Abare, P. Kozodoy, T.M. Katona, M.D. Craven, J.S. Speck,U.K. Mishra, L.A. Coldren, and S.P. DenBaars, phys. stat. sol. (a) 176, 59 (1999).

[Heon, 1995] Lee Heon, Oberman David B,Harris ,James S Jr, Appl.Phys.Lett, Vol 67.Issue12,September18 1995 , pp.1754-1756.

[Holonyak, 1980] Nick Holonyak Jr,Robert M Kolbas,Daniel Dapkus,IEEE J.Quantum.Elec, VO1,QE-16,No 2,February 1980.

[Itaya, 1996]K. Itaya, M. Onomura, J. Nishio, L. Sugiura, S. Saito, M. Suzuki, J. Rennie, S. Nunoue, M. Yamamato, H. Fujimoto, Y. Kokobun, Y. Ohba, G. Hatakoshi, M. Ishikawa ,Jap.J.App.Phys 35(10B), 11315 (1996).

[Krestnikov, 2002] I. L. Krestnikov, N. N. Ledentsov, A. Hoffmann, and D. Bimberg, A. V. Sakharov, W. V. Lundin, A. F. Tsatsul'nikov, A. S. Usikov, and Zh. I. Alferov, A.F. Ioffe, Yu. G. Musikhin and D. Gerthsen, Phys. Rev B 66, 1553102002.

[Kuo, 2004] Yen-Kuang Kuo, Yi-An Chang, IEEE.J.Quantum.Elec, Vol. 40, NO. 5, May 2004. 
[Kuramata, 1999] Akito Kuramata, Shin-ichi Kubota, Reiko Soejima, Kay Domen, Kazuhiko Horino, Peter Hacke and Toshiyuki Tanahashi, Jpn. J. Appl. Phys,Vol.38(1999)L481-L483 Part 2, No. 5A, 1 May 1999.

[Lester, 1995] S. D. Lester, F. A. Ponce, M. G. Craford and D. A. Steigerwald, 1995 American. Inst. Phys.

[Look, 2004] D. C. Look,B. Claflin, Ya. I. Alivov, and S. J. Park phys.stat.sol.(a)201,No.10,2203-2212(2004)/DOI002/pssa. 200404803.

[Martin, 2002] RW Martin, P R Edwards, R Pecharroman-Gallego, C Liu,C J Deatcher, I M Watson and K P O’Donnell, Inst.Phys Publishing J. Phys, Appl.Phys, J.Phys.D:Appl.Phys, 35(2002)604-608PII:S0022-3727(02)31329-9.

[Maruyama, 2001] T. Maruyama, Y. Hagio ,T. Miyajima , S. Kijima, Y. Nanishi , K. Akimoto, Noji-Higashi, Kusatsu, Ten-nodai, Tsukuba, Ohsaki, Shinagawa-ku, phys. stat. sol. (a) 188, No. 1, 375-378 (2001).

[Monroy, 2003] E. Monroy, F. Omnes, F, Calle, Semicond. Sci. Technol. R33-R51, 18, 2003.

[Nagahama, 2001] Shinichi Nagahama, Naruhito Iwasa, Masayuki Senoh, Toshio Matsushita, Yasunobu Sugimoto, Hiroyuki Kiyoku, Tokuya Kozaki, Masahiko Sano, Hiroaki Matsumura, Hitoshi Umemoto, Kazuyuki Chocho, and Takashi Mukai, Proceedings of SPIE -- Volume 4287.

[Nakamura, 1996] S Nakamura,S Nakamura, M Senoh, S Nagahama, N Iwasa, T Yamada, T Matsushita, H Kiyoku, Y Sugimoto, Jap.J.App.Phys 35(1B), L74 (1996).

[Nakamura, 1996] S Nakamura,S Nakamura, M Senoh, S Nagahama, N Iwasa, T Yamada, T Matsushita, H Kiyoku, Y Sugimoto, Jap.J.App.Phys 35(2B), L217 (1996). 
[Nakamura, 1996] Shuji Nakamura,Masayuki Senoh, Shin-ichi Nagahama, Naruhito Iwasa, Takao Yamada, ToshioMatsushita, Hiroyuki Kiyoku, Yasunobu Sugimoto , Appl. Phys. Lett 68(15), 2105 (1996).

[Nakamura, 1996]Shuji Nakamura, Masayuki Senoh, Shin-ichi Nagahama, Naruhito Iwasa, Takao Yamada, Toshio Matsushita, Hiroyuki Kiyoku, Yasunobu Sugimoto,App.Phys.Lett 68(23), 3269 (1996).

[Nakamura, 1996] Shuji. Nakamura ,M. Senoh, S. Nagahama, N. Iwasa, T. Yamada, T. Matsushita, Y. Sugimoto, H. Kiyoku ,App.Phys.Lett 69(10), 1477 (1996).

[Nakamura, 1996] Shuji. Nakamura, M. Senoh, S. Nagahama, N. Iwasa, T. Yamada, T. Matsushita, Y. Sugimoto, H. Kiyoku,App.Phys.Lett 69, 3034 (1996).

[Nakamura, 1996] Shuji. Nakamura, M. Senoh, S. Nagahama, N. Iwasa, T. Yamada, T. Matsushita, Y. Sugimoto, H. Kiyoku ,App.Phys.Lett 69(26), 4056 (1996).

[Narukawa, 1997]Yukio Narukawa,Yoichi Kawakami,a) Mitsuru Funato, Shizuo Fujita, and Shigeo Fujita, Appl. Phys. Lett., 70(8):981-3, 24 February 1997

[Nakamura, 1998] Shuji Nakamura,Masayuki Senoh, Shin-ichi Nagahama, Naruhito Iwasa, Takao Yamada, Toshio Matsushita, Hiroyuki Kiyoku, Yasunobu Sugimoto, Tokuya Kozaki, Hitoshi Umemoto, Masahiko Sano, and Kazuyuki Chocho, Appl.Phys. Lett 72(16), 2014 (1998).

[Narayana, 2002] J. Narayana, H. Wang, Appl.Phys Lett ,Vol 81, No 5.

[Obata, 2004] Toshiyuki Obata, Hideki Hirayama, Yoshinobu Aoyagi, Koji Ishibashi, phys. stat. sol. (a) 201, No. 12, 2803-2807 (2004) / DOI 10.1002/pssa.200405120.

[Ozgur, 2001] Umit Ozgur, Grady Webb-Wood, and Henry O. Everitt, Feng Yun and Hadis Morkoc, Appl.Phys,Lett,Vol 79 No 2517 December 2001. 
[Piprek, 2000] Joachim Piprek, R. Kehl Sink a, Monica A. Hansen b, John E. Bowers , and Steve P. DenBaars b, Phys and Sim.Opto.Dev VIII, ed. R. Binder, P. Blood \& M. Osinski, SPIE Proc. 3944, 2000.

[Ryan, 1999] M. E. Ryan, A. C. Camacho, J. K. Bhardwaj, phys. stat. sol. (a) 176, 743 (1999).

[Song, 2004] June-O Song, Dong-Seok Leem, Sang-Ho Kim, J. S. Kwak, O. H. Nam, Y. Park and Tae-Yeon Seong, Solid-State.Elec 48 (2004) 1597-1600.

[Stocker, 1998] D. A. Stocker and E. F. Schubert, J. M. Redwing, Appl.Phys.Lett, Vol 73, No 18, 2 November 1998.

[Streetman, 2000] Ben G.Streetman, Sanjay Banerjee,Pearson Education, Inc 2000.

[Torvik, 1999] John T. Torvik, Jacques I. Pankove, Bart J. Van Zeghbroeck, IEEE Transactions on Electron Devices, 46 (7), 1999.

[Ueta, 2004] A.Ueta, M. Klude, A. Gust, S. Figge, K. Otte, and D. Hommel , phys. stat. sol. (c) 1, No. 4, 1014-1017 (2004) / DOI 10.1002/pssc.200304123.

[Wang, 1999] T.Wang, D. Nakagawa, M. Lachab, T. Sugahara, and S. Sakai, Appl.Phys.Lett, Vol 74 No21 24 May 1994.

[Weizong, 2002] Chen Weizong, Bo Tao; Fu Lingli; Zheng Xinliang; Li Shaoxiong; Zhou Jinghui, Acta Photonica Sinica, v 31, n 9, Sept. 2002, p 1124-7.

[Williams, 1996] Kirt R.Williams , Kishan Gupta, Matthew Wasilik, J. Microelectromechanical.Sys, Vol 5 ,No 4, December. 
[Wilmers, 1999] K. Wilmers, T. Wethkamp,N. Esser,C. Cobet,W. Richter,V. Wagner,H. Lugauer,F. Fischer,T. Gerhard M. Keim,and M. Cardona, J.Elec.Materials, Vol. 28, No. 6, 1999.

[Zhou, 2000] L. Zhou,L. Zhou,W. Lanford, A. T. Ping, I. Adesida, J. W. Yang ,A. Khan, App.Phys.Lett, Vol 76, No 235 June 2000.

[Zhu, 2004] K. Zhu, M. L. Nakarmi, K. H. Kim, J. Y. Lin, and H. X. Jiang, Appl.Phys.Lett, Vol 85, No20, 15 November 2004. 


\section{APPENDIX A: SOLUTION FOR INDIUM MOLE FRACTION OF THE GRADED ACTIVE REGION-USING MATLAB PROGRAM}

clear all;

clc;

global Vo1;

Potential well depth in e.v.

global a;

Thickness of the quantum well

Calculating the emission energy of the first quantum well

xo = input ('In Enter the indium mole fraction of the first quantum well: ');

if $\mathrm{xo}>=0.01 \& \& \mathrm{xo}<0.06458$

$\mathrm{b} 1=(-6.86486 * \mathrm{xo})+2.51425 ;$

elseif xo $>=0.06458 \& \&$ xo $<0.0937$

$$
\mathrm{b} 1=(-4.44 * \mathrm{xo})+2.3581
$$

Calculating the bowing parameter of

elseif $\mathrm{xo}>=0.0937 \& \& \mathrm{xo}<0.25$ InGaN for a particular Indium mole

$\mathrm{b} 1=(-2.635 * \mathrm{xo})+2.1887$; fraction. [Ferhat, 2001]

else $\mathrm{xo}>=0.25 \& \& \mathrm{xo}<0.5$

$\mathrm{b} 1=(-0.8 * \mathrm{xo})+1.8$

end

xo1 = input ('In Enter the indium mole fraction of barrier : ');

if $\mathrm{xo} 1>=0.01 \& \&$ xo $1<0.06458$

$$
\mathrm{b} 2=(-6.86486 * \mathrm{xo} 1)+2.51425 \text {; }
$$

elseif xo1 $>=0.06458 \& \&$ xo $1<0.0937$

$$
\text { b2 }=(-4.44 * x o 1)+2.3581 \text {; }
$$

elseif xo1 $>=0.0937 \& \&$ xo $1<0.25$

$$
\mathrm{b} 2=(-2.635 * \mathrm{xo} 1)+2.1887
$$




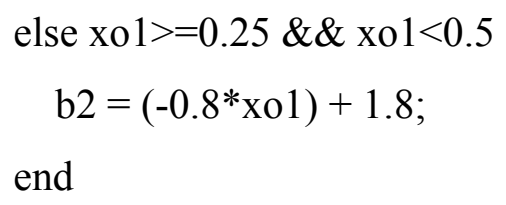
an indium mole fraction xo1. [Binder, 2000].

Vo1=0.3*(Eg1-Eg);

Calculating potential well depth in the Conduction band of the first quantum well Band offset: 0.3[Binder, 2000]

$\mathrm{mo}=9.11 * 10^{\wedge}-31$

eme $=0.151 * \mathrm{mo}+\mathrm{xo} *(0.1 * \mathrm{mo}-0.151 * \mathrm{mo})$ Calculating effective mass of electrons [Kuo, 2004]

$\mathrm{c} 0=\mathrm{Vo}$;

$\mathrm{i}=1$;

Solving for the discrete energy levels in the conduction band of the first quantum well.

Computing the tan part of the solution 


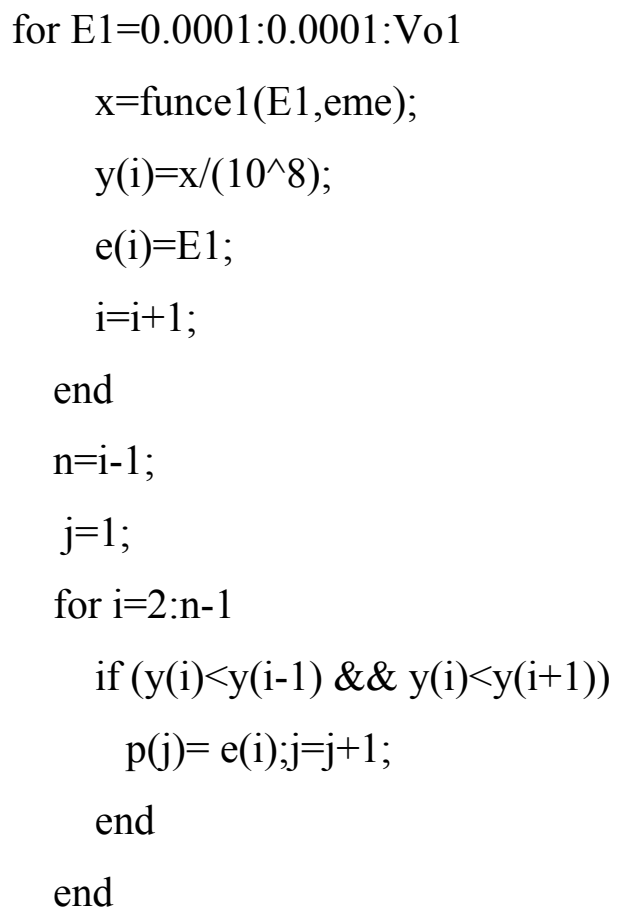

This is to compute the cot part of the solution

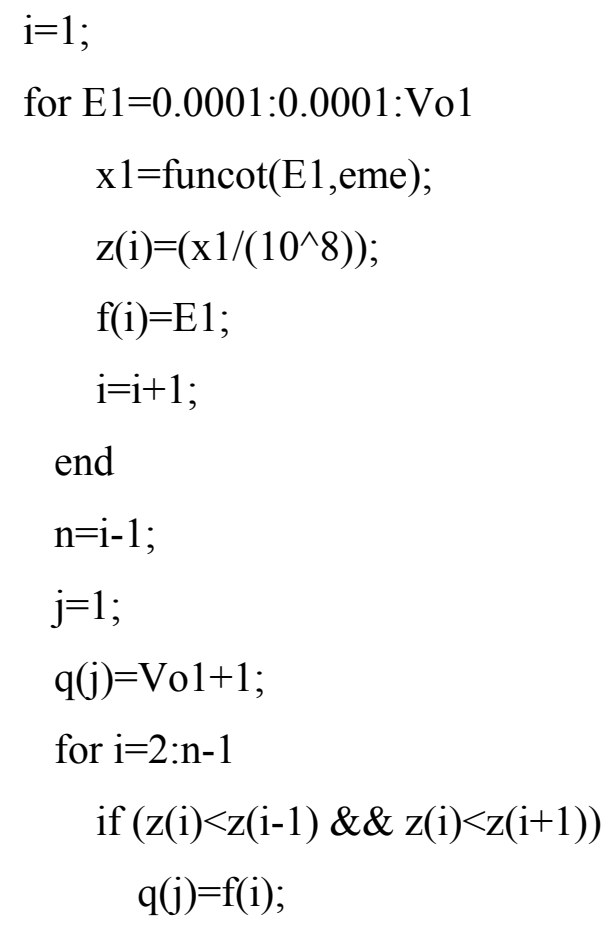




$$
\begin{aligned}
& \mathrm{j}=\mathrm{j}+1 \text {; } \\
& \text { end } \\
& \text { end } \\
& \text { if } p(1)>q(1) \\
& \mathrm{e} 1=\mathrm{q}(1) ; \\
& \text { else } \mathrm{e} 1=\mathrm{p}(1) \text {; } \\
& \text { end } \\
& \mathrm{c} 1=\mathrm{p} \text {; } \\
& \mathrm{c} 2=\mathrm{q} \text {; } \\
& \mathrm{c} 3=\mathrm{e} 1 \text {; }
\end{aligned}
$$

Solving for discrete energy levels in the valence band of the first quantum well.

$$
\begin{aligned}
\mathrm{eme}=\mathrm{mo} * 1.595+\mathrm{xo} *(1.449-1.595) * \mathrm{mo} ; & \text { Calculating effective mass of heavy } \\
& \text { holes }[\text { Kuo, 2004] }
\end{aligned}
$$

Vo1=0.7*(3.45-Eg);

$$
\begin{aligned}
& \text { Calculating potential well depth in the } \\
& \text { valence band of the first quantum well } \\
& \text { Band offset: 0.7[Binder, 2000] }
\end{aligned}
$$

This is to compute the tan part of the solution

$$
\begin{aligned}
& \mathrm{i}=1 ; \\
& \text { for } \mathrm{E} 1=0.0001: 0.0001: \text { Vo1 } \\
& \qquad \begin{array}{l}
\mathrm{x}=\text { funce1 }(\mathrm{E} 1, \mathrm{eme}) ; \\
\mathrm{y}(\mathrm{i})=\mathrm{x} /\left(10^{\wedge} 8\right) ;
\end{array}
\end{aligned}
$$




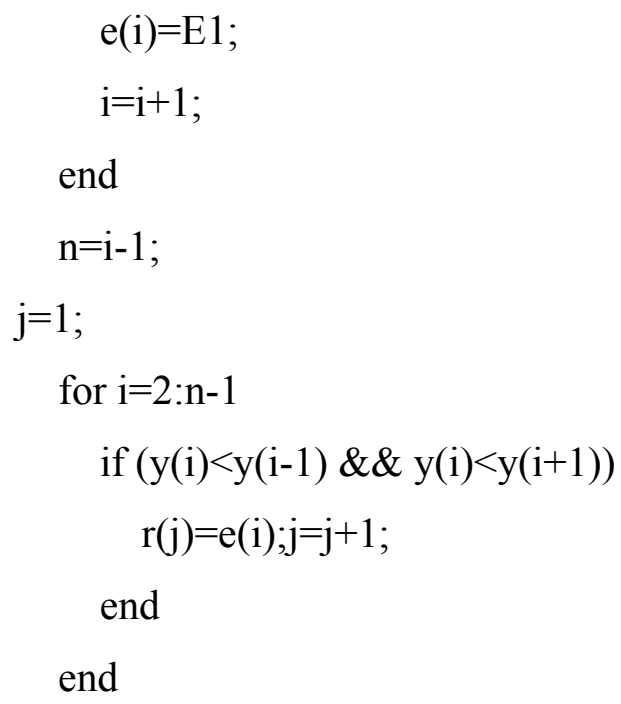

This is to compute the cot part of the solution

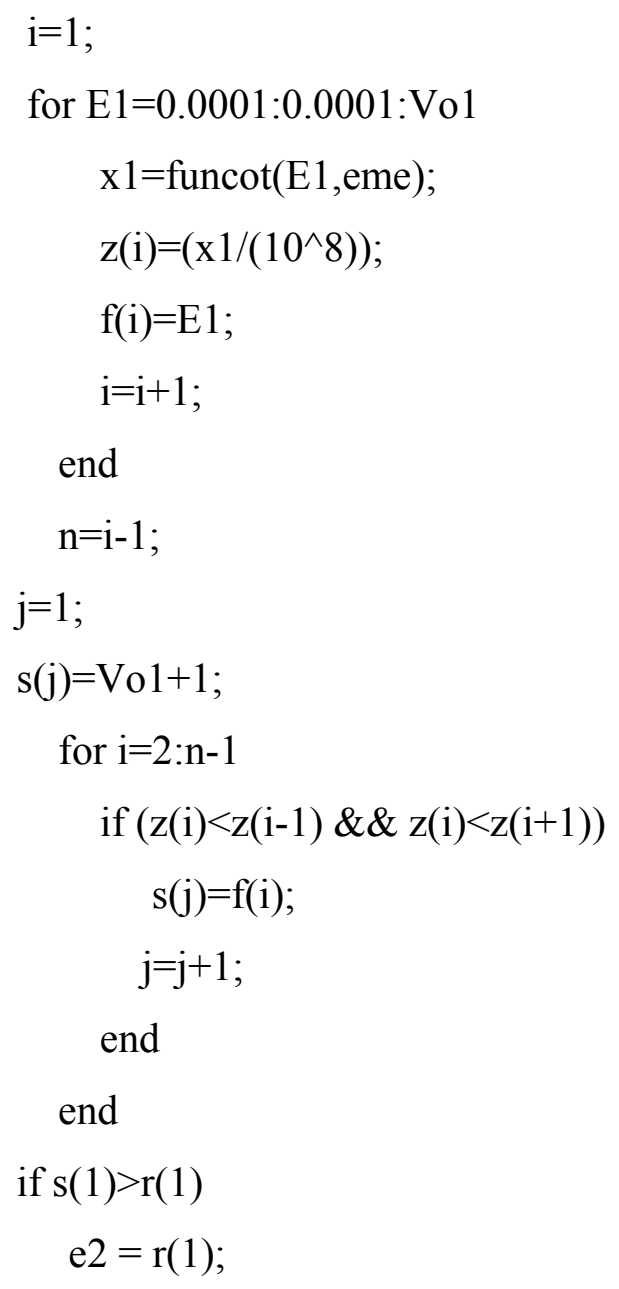


else e $2=\mathrm{s}(1)$

end

$\mathrm{c} 4=\mathrm{r}$;

$\mathrm{c} 5=\mathrm{s}$;

$\mathrm{c} 6=\mathrm{e} 2$;

$\mathrm{Eg}$;

$\mathrm{E} 0=\mathrm{Eg}+\mathrm{e} 1+\mathrm{e} 2$

Effective energy gap of the first quantum well.

Determining the indium mole fraction of the second quantum well for a given thickness of the second quantum well for the same emission wavelength as the first quantum well

xo1 = input ('In Enter the indium mole fraction of the barrier: '); Same as the first barrier.

$\mathrm{a}=$ input ("In Enter the thickness of second quantum well in nm: ');

if $\mathrm{xo} 1>=0.01 \& \& \mathrm{xo} 1<0.06458$

$\mathrm{b} 2=(-6.86486 * x o 1)+2.51425$;

elseif xo1 $>=0.06458 \& \&$ xo $1<0.0937$

b2 $=(-4.44 * x 01)+2.3581$;

elseif xo $1>=0.0937 \& \&$ xo $1<0.25$

$$
\mathrm{b} 2=\left(-2.635^{*} \mathrm{xo} 1\right)+2.1887 \text {; }
$$

else $\mathrm{xo} 1>=0.25 \& \&$ xo $1<0.5$

$$
\mathrm{b} 2=(-0.8 * \mathrm{xo} 1)+1.8
$$

end

$\mathrm{Eg} 1=\mathrm{xo} 1 * 1.9+(1-\mathrm{xo} 1) * 3.45-\mathrm{b} 2 * \mathrm{xo} 1 *(1-\mathrm{xo} 1)$ 


$\begin{array}{ll}\text { for } \mathrm{x} 2=\mathrm{xo}:-0.00001: 0 & \text { Calculating indium mole fraction } \\ & \text { at which the emission energy of } \\ & \text { the first quantum well is almost } \\ & \text { equal to the emission energy of } \\ & \text { second quantum well. }\end{array}$
if $(\mathrm{x} 2>=0.01 \& \& \mathrm{x} 2<0.06458)$
$\mathrm{b} 1=(-6.86486 * \mathrm{x} 2)+2.51425$;
elseif $(\mathrm{x} 2>=0.06458 \& \& \mathrm{x} 2<0.0937)$
$\mathrm{b} 1=(-4.44 * \mathrm{x} 2)+2.3581$;
elseif $(\mathrm{x} 2>=0.0937 \& \& \mathrm{x} 2<0.25)$
$\mathrm{b} 1=(-2.635 * \mathrm{x} 2)+2.1887$;
else $(\mathrm{x} 2>=0.25 \& \& \mathrm{x} 2<0.5)$
$\mathrm{b} 1=(-0.8 * \times 2)+1.8$
end

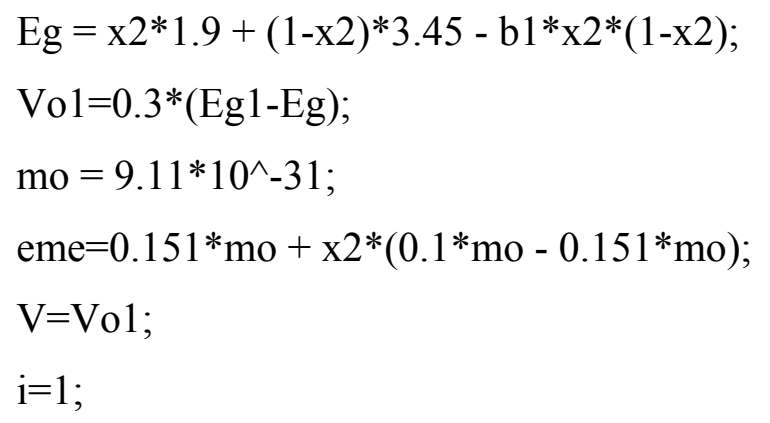

Solving for discrete energy level in the conduction band of the second quantum well.

This is to compute the tan part of the solution

$$
\text { for } \begin{aligned}
\mathrm{E} 1 & =0.0001: 0.0001: \text { Vo1 } \\
\mathrm{x} & =\text { funce1(E1,eme) }
\end{aligned}
$$




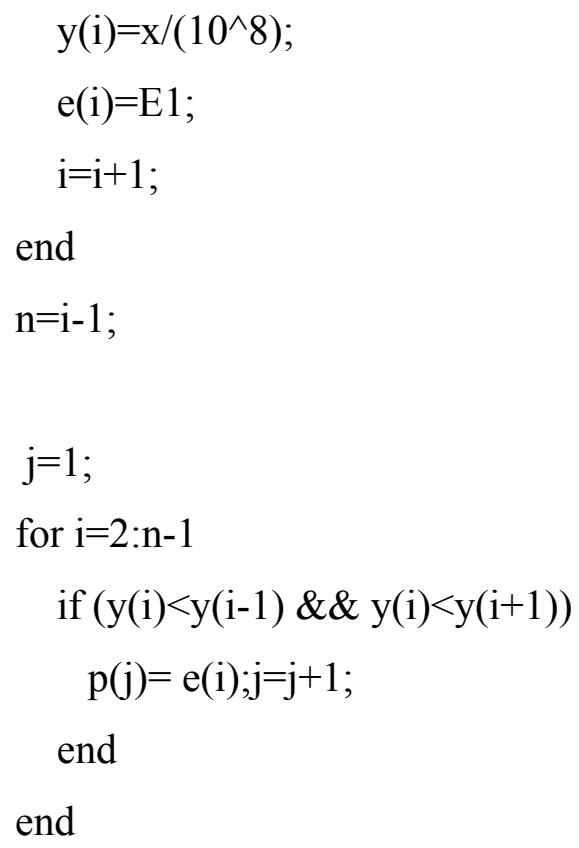

This is to compute the cot part of the solution

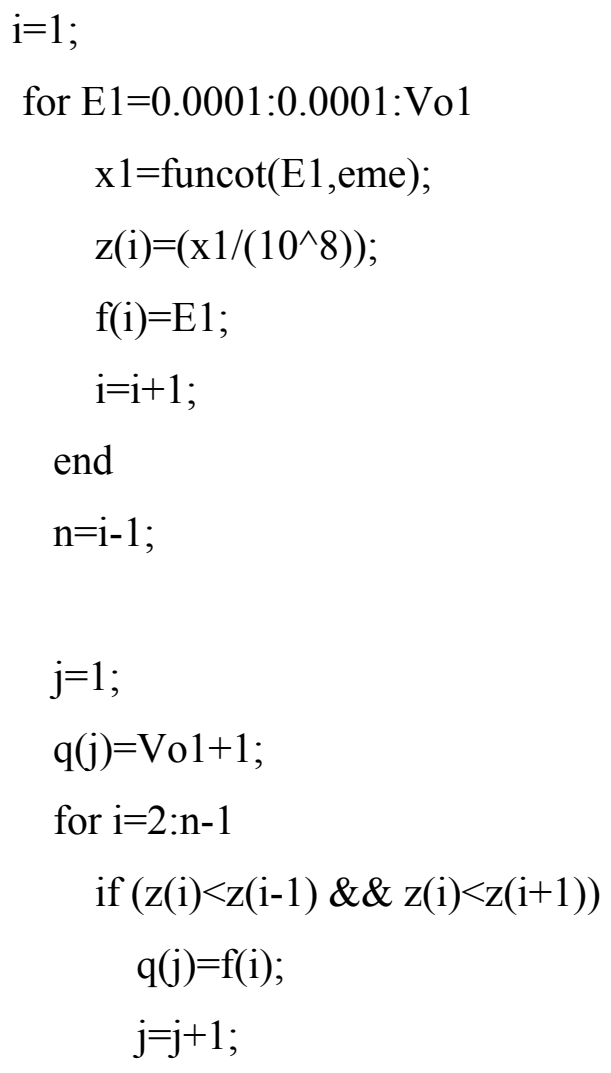




$$
\begin{aligned}
& \text { end } \\
& \text { end } \\
& \text { if }(p(1)>q(1)) \\
& \quad \text { e1 }=q(1) ; \\
& \text { else e } 1=p(1) ; \\
& \text { end } \\
& \text { ee }=\mathrm{e} 1 ; \\
& \text { E1=0.0001:0.0001: Vol; }
\end{aligned}
$$

Solving for discrete energy levels in the valence band of the second quantum well

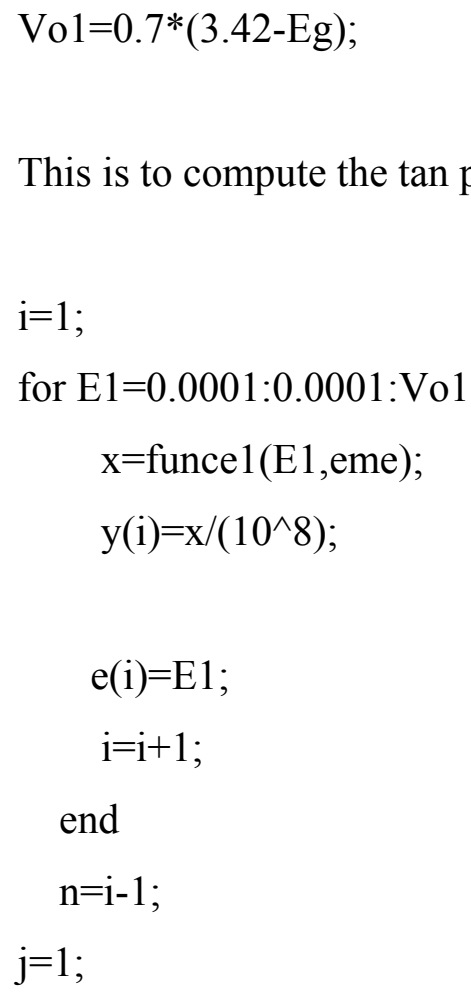


well at a particular Indium mole fraction.

$\mathrm{c}=\mathrm{E} 0-\mathrm{E} 01 ;$

$\operatorname{if}(\mathrm{c}<=0.000005)$

Mole fraction of indium at which

emission energy of both the quantum

wells is almost equal

fprintf('The Potential barrier in the conduction band of the second quantum well : \%f $\left\lfloor n^{\prime}, \mathrm{V}\right)$;

fprintf('The Potential barrier in the conduction band is first quantum well : \% $\backslash \mathrm{n}^{\prime}, \mathrm{c} 0$ );

fprintf('The lowest energy level in the second quantum well : \%f $\backslash n$ ',ee);

fprintf('The lowest energy level in the first quantum well: \%f $\backslash \mathrm{n}$ ',c3);

fprintf('The indium mole fraction in the second quantum well : \% $\backslash \mathrm{n}$ ', $\mathrm{x} 2)$;

break;

end

end

\section{FUNCTIONS USED IN THE ABOVE PROGRAM}

The function funce1() gives the tangent part of the solution

function funcval $=$ funce $1(\mathrm{E} 1, \mathrm{eme})$;

eme;

global Vo1;

global a;

ap $=\operatorname{sqrt}\left(\left(2 * \mathrm{eme}^{*} \mathrm{E} 1 * 1.6\right) /\left(1.113 * 10^{\wedge}-68 * 10^{\wedge} 19\right)\right)$; 


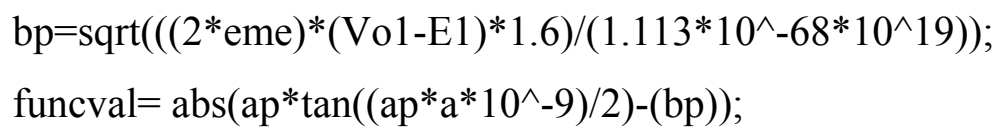

The function funcot() gives the cotangent part of the solution

function funcval $=$ funcot $(\mathrm{E} 1, \mathrm{eme})$;

eme;

global Vo1;

global a;

$\mathrm{ap}=\operatorname{sqrt}\left(\left(2 * \mathrm{eme}^{*} \mathrm{E} 1 * 1.6\right) /\left(1.113 * 10^{\wedge}-68 * 10^{\wedge} 19\right)\right)$;

$\mathrm{bp}=\operatorname{sqrt}\left(((2 * \mathrm{eme}) *(\operatorname{Vo} 1-\mathrm{E} 1) * 1.6) /\left(1.113 * 10^{\wedge}-68^{*} 10^{\wedge} 19\right)\right)$;

funcval $=\operatorname{abs}\left(a p^{*} \cot \left(\left(a p^{*} a^{*} 10^{\wedge}-9\right) / 2\right)+(b p)\right)$; 


\section{APPENDIX B - SOLUTION FOR THE LOWEST ENERGY IN THE CONDUCTION BAND OF THE WIDER QUANTUM WELL IN A DOUBLE BARRIER QUANTUM WELL-USING MATLAB PROGRAM}

clear

clear all;

clc;

global Vo

global a

global w

xo = input('In Enter the indium mole fraction of the quantum well: ');

$\mathrm{a}=\operatorname{input}($ 'In Enter the quantum well thickness: ');

$\mathrm{w}=\operatorname{input}(' \mathrm{In}$ Enter the thickness of the first barrier : ');

if $\mathrm{xo}>=0.01 \& \& \mathrm{xo}<0.06249$

$$
\mathrm{b} 1=(-10.64 * \mathrm{xo})+2.72377 \text {; }
$$

elseif xo $>=0.06249 \& \&$ xo $<0.0928564$

$$
\mathrm{b} 1=(-3.787 * \mathrm{xo})+2.2916 \text {; }
$$

elseif xo $>=0.0928564 \& \&$ xo $<0.25$

$$
\mathrm{b} 1=(-2.48 * \mathrm{xo})+2.17062
$$

elseif $\mathrm{xo}>=0.25 \& \&$ xo $<0.5$

$$
\mathrm{b} 1=(-0.4 * \mathrm{xo})+1.65
$$

end

xo1 = input('In Enter the indium mole fraction xo1 of the first barrier: ');

if $\mathrm{xo} 1>=0.01 \& \&$ xo $1<0.06249$ 


$$
\begin{aligned}
& \text { b2 }=(-10.64 * x o 1)+2.72377 \text {; } \\
& \text { elseif xo } 1>=0.06249 \& \& \text { xo } 1<0.0928564 \\
& \text { b2 }=(-3.787 * x o 1)+2.2916 \text {; } \\
& \text { elseif xo1 }>=0.0928564 \& \& \text { xo } 1<0.25 \\
& \text { b2 }=(-2.48 * x o 1)+2.17062 \text {; } \\
& \text { elseif } \text { xo } 1>=0.25 \& \& \text { xo } 1<0.5 \\
& \text { b2 }=(-0.4 * x o 1)+1.65 \text {; } \\
& \text { elseif } \text { xo1 }==0 \\
& \mathrm{~b} 2=0 \text {; }
\end{aligned}
$$

xo2 $=$ input('\n Enter the indium mole fraction xo1 of the second barrier: ');

if $\mathrm{xo} 2>=0.01 \& \&$ xo $2<0.06249$

$$
\text { b3 }=(-10.64 * x o 1)+2.72377 \text {; }
$$

elseif xo $2>=0.06249 \& \&$ xo $2<0.0928564$

$$
\text { b3 }=(-3.787 * \text { xo2 })+2.2916 \text {; }
$$

elseif xo2 $>=0.0928564 \& \&$ xo $2<0.25$

$$
\mathrm{b} 3=(-2.48 * \mathrm{xo} 2)+2.17062 \text {; }
$$

elseif xo $1>=0.25 \& \&$ xo $1<0.5$

$$
\text { b3 }=(-0.4 * x 02)+1.65
$$

elseif хо $2=0$

$$
\text { b3 }=0 \text {; }
$$

end

$$
\begin{aligned}
& \mathrm{Eg}=\mathrm{xo}^{*} 1.9+(1-\mathrm{xo}) * 3.45-\mathrm{b} 1 * \mathrm{xo} *(1-\mathrm{xo}) \\
& \mathrm{Eg} 1=\mathrm{xo} 1 * 1.9+(1-\mathrm{xo} 1) * 3.45-\mathrm{b} 2 * \mathrm{xo} 1 *(1-\mathrm{xo} 1) \\
& \mathrm{Eg} 2=\mathrm{xo} 2 * 1.9+(1-\mathrm{xo} 2) * 3.45-\mathrm{b} 3 * \mathrm{xo} 2 *(1-\mathrm{xo} 2) \\
& \mathrm{Vo} 2=0.3 *(\mathrm{Eg} 2-\mathrm{Eg} 1) \\
& \mathrm{Vo}=\mathrm{Vo} 2 \\
& \mathrm{mo}=9.11 * 10^{\wedge}-31
\end{aligned}
$$

Appendix A

Appendix A 


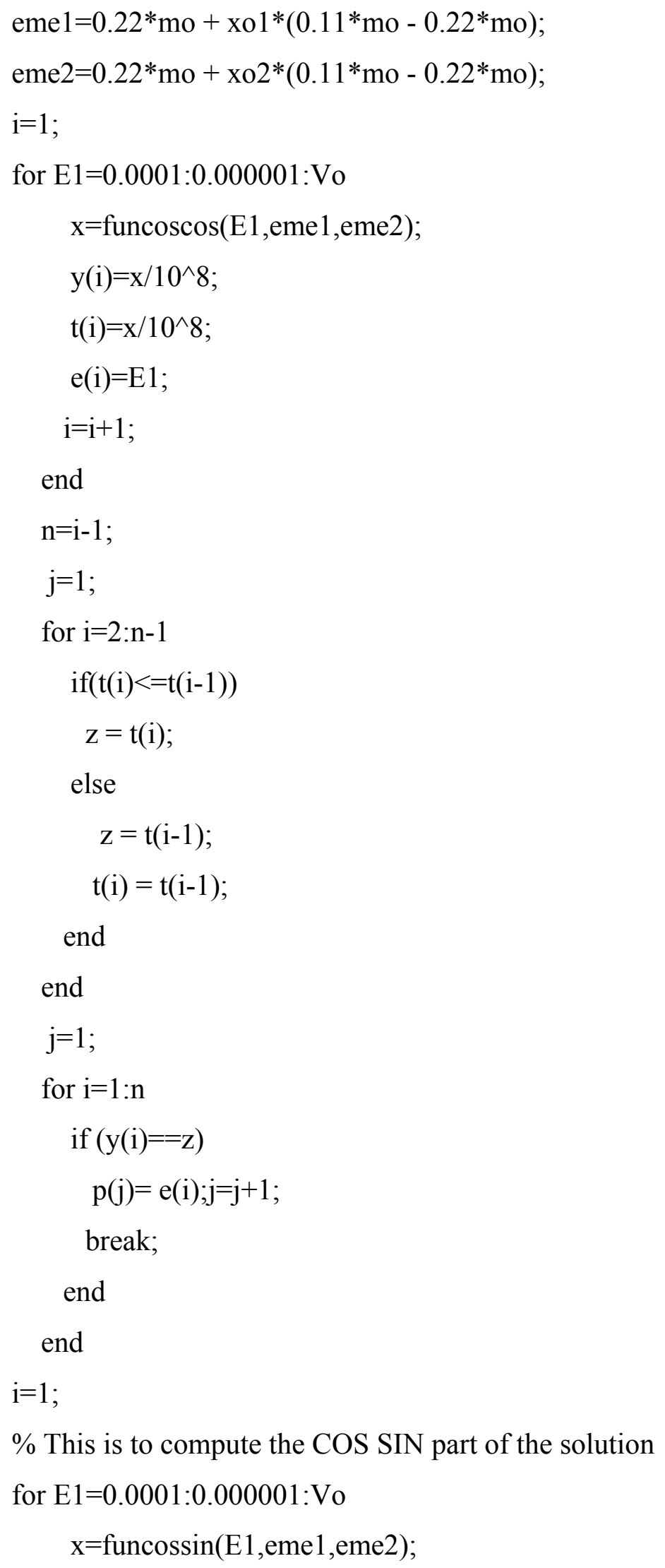




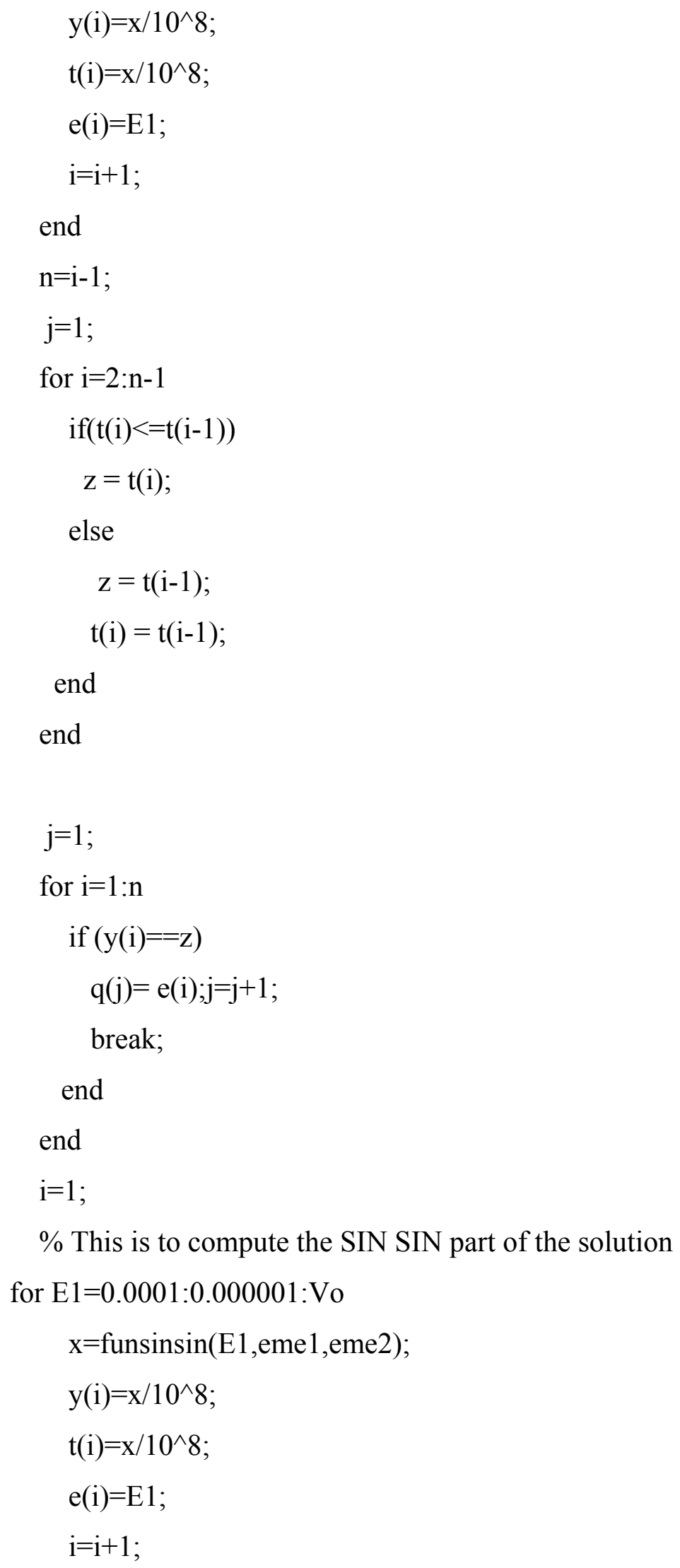




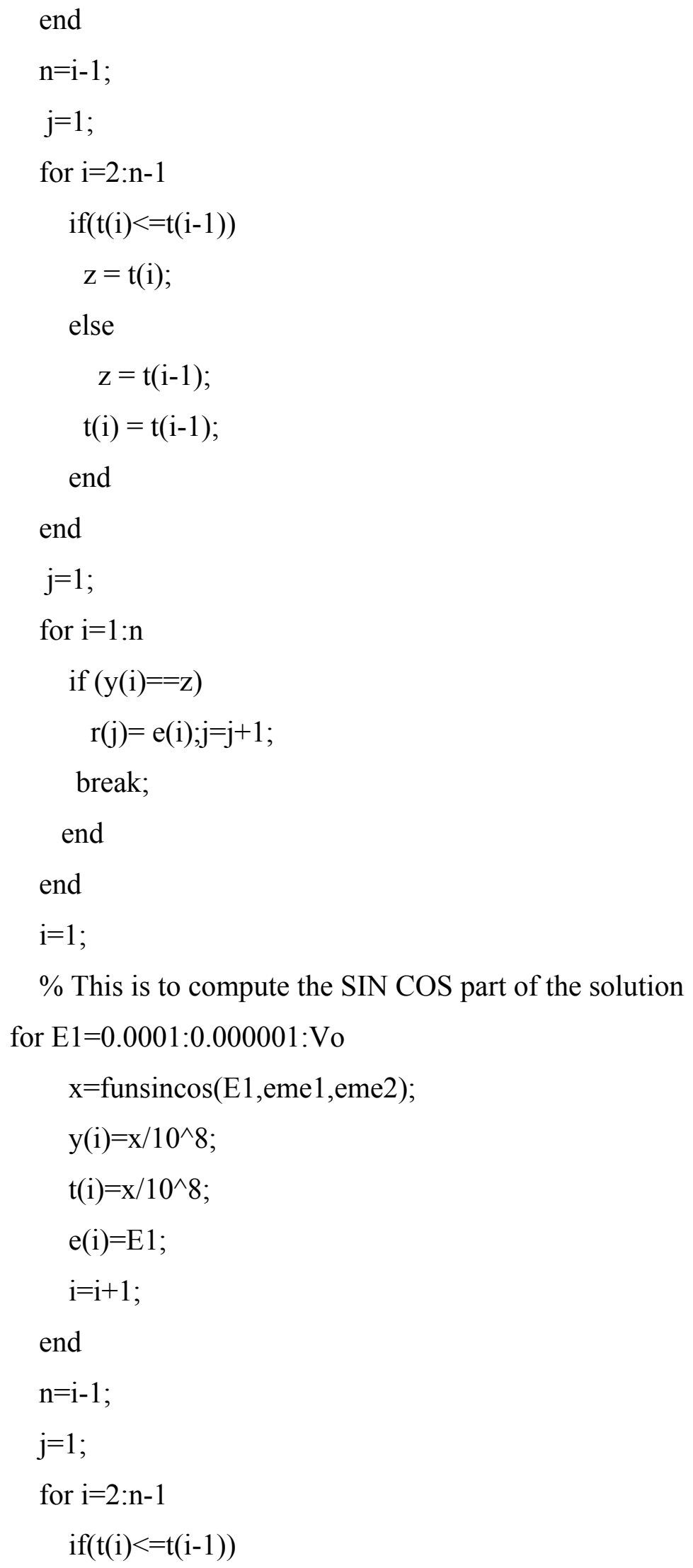




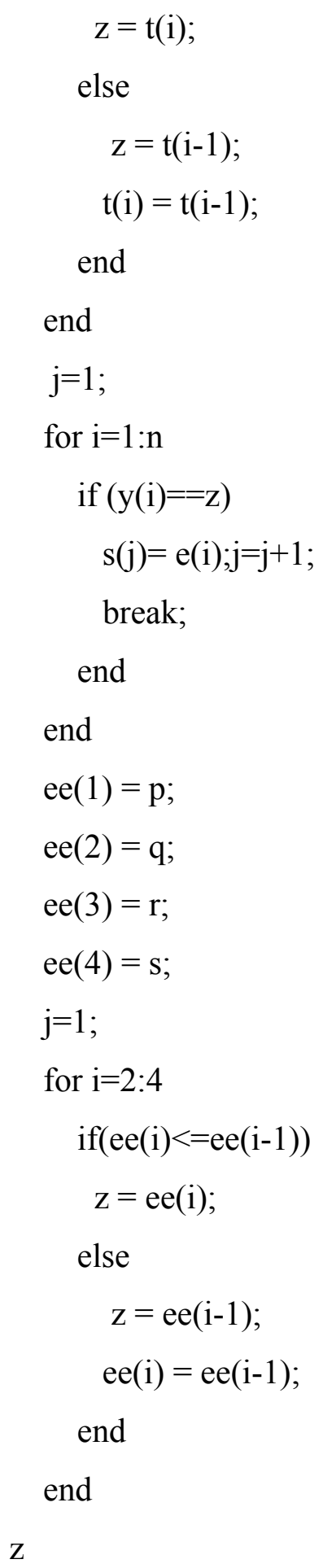




\section{FUNCTIONS USED IN THE ABOVE PROGRAM}

The funsinsin() is used to calculate the sinsin part.

function funcval $=$ funsinsin(E1,eme1,eme2);

global Vo;

global a;

global w;

$\mathrm{k} 1=\operatorname{sqrt}\left((2 * \mathrm{eme} 1 * \mathrm{E} 1 * 1.6) /\left(1.113^{*} 10^{\wedge}-68^{*} 10^{\wedge} 19\right)\right)$;

$\mathrm{k} 2=\operatorname{sqrt}\left((2 * \mathrm{eme} 2 * \mathrm{E} 1 * 1.6) /\left(1.113 * 10^{\wedge}-68^{*} 10^{\wedge} 19\right)\right)$;

$\mathrm{k} 3=\operatorname{sqrt}\left(((2 *\right.$ eme 2$) *($ Vo-E1 $\left.) * 1.6) /\left(1.113 * 10^{\wedge}-68 * 10^{\wedge} 19\right)\right)$;

funcval $=\operatorname{abs}((\mathrm{k} 3 * \tan (\mathrm{k} 2 * \mathrm{w} / 2) * \cot (\mathrm{k} 2 * \mathrm{a} / 2))+(\mathrm{k} 1 * \cot (\mathrm{k} 1 * \mathrm{a} / 2)))$;

The funsincos() is used to calculate the sincos part.

function funcval $=$ funsincos $(\mathrm{E} 1, \mathrm{eme} 1, \mathrm{eme} 2)$;

global Vo;

global a;

global w;

$\mathrm{k} 1=\operatorname{sqrt}\left((2 * \mathrm{eme} 1 * \mathrm{E} 1 * 1.6) /\left(1.113^{*} 10^{\wedge}-68^{*} 10^{\wedge} 19\right)\right)$;

$\mathrm{k} 2=\operatorname{sqrt}\left((2 * \mathrm{eme} 2 * \mathrm{E} 1 * 1.6) /\left(1.113 * 10^{\wedge}-68^{*} 10^{\wedge} 19\right)\right)$;

$\mathrm{k} 3=\operatorname{sqrt}\left(((2 * \mathrm{eme} 2) *(\mathrm{Vo}-\mathrm{E} 1) * 1.6) /\left(1.113 * 10^{\wedge}-68^{*} 10^{\wedge} 19\right)\right)$;

funcval $=\operatorname{abs}((\mathrm{k} 3 * \cot (\mathrm{k} 2 * \mathrm{w} / 2) * \tan (\mathrm{k} 2 * \mathrm{a} / 2))+(\mathrm{k} 1 * \cot (\mathrm{k} 1 * \mathrm{a} / 2)))$;

The funcossin() is used to calculate the cossin part

function funcval $=$ funcossin(E1,eme1,eme $)$;

global Vo;

global a;

global w;

$\mathrm{k} 1=\operatorname{sqrt}\left((2 * \mathrm{eme} 1 * \mathrm{E} 1 * 1.6) /\left(1.113 * 10^{\wedge}-68 * 10^{\wedge} 19\right)\right)$;

$\mathrm{k} 2=\operatorname{sqrt}\left((2 * \mathrm{eme} 2 * \mathrm{E} 1 * 1.6) /\left(1.113 * 10^{\wedge}-68^{*} 10^{\wedge} 19\right)\right)$;

$\mathrm{k} 3=\operatorname{sqrt}\left(((2 * \mathrm{eme} 2) *(\mathrm{Vo}-\mathrm{E} 1) * 1.6) /\left(1.113 * 10^{\wedge}-68^{*} 10^{\wedge} 19\right)\right)$;

funcval $=\operatorname{abs}((\mathrm{k} 3 * \tan (\mathrm{k} 2 * \mathrm{w} / 2) * \cot (\mathrm{k} 2 * \mathrm{a} / 2))-(\mathrm{k} 1 * \tan (\mathrm{k} 1 * \mathrm{a} / 2)))$; 
The funcoscos() is used to calculate the $\operatorname{coscos}$ part function funcval $=$ funcoscos $(\mathrm{E} 1$,eme1,eme2);

global Vo;

global a;

global w;

$\mathrm{k} 1=\operatorname{sqrt}\left((2 * \mathrm{eme} 1 * \mathrm{E} 1 * 1.6) /\left(1.113^{*} 10^{\wedge}-68^{*} 10^{\wedge} 19\right)\right)$;

$\mathrm{k} 2=\operatorname{sqrt}\left((2 * \mathrm{eme} 2 * \mathrm{E} 1 * 1.6) /\left(1.113^{*} 10^{\wedge}-68^{*} 10^{\wedge} 19\right)\right)$;

$\mathrm{k} 3=\operatorname{sqrt}\left(\left((2 * \mathrm{eme} 2)^{*}(\mathrm{Vo}-\mathrm{E} 1)^{*} 1.6\right) /\left(1.113^{*} 10^{\wedge}-68^{*} 10^{\wedge} 19\right)\right)$;

funcval $=\operatorname{abs}((\mathrm{k} 3 * \cot (\mathrm{k} 2 * \mathrm{w} / 2) * \tan (\mathrm{k} 2 * \mathrm{a} / 2))-(\mathrm{k} 1 * \tan (\mathrm{k} 1 * \mathrm{a} / 2)))$; 


\section{APPENDIX C:SOLUTION TO 1D TIME-INDEPENDENT SCHRODINGER WAVE EQUATION USING MATLAB}

clear all;

clc;

global Vo1

global a

$\mathrm{a}=\operatorname{input}(' \mathrm{n}$ Enter the thickness of well: ');

xo1 = input('In Enter the value of indium mole fraction xo1 of barrier: ');

if $x o 1>=0.01 \& \&$ xo $1<0.06249$

b2 $=(-10.64 * x o 1)+2.72377$;

elseif xo $1>=0.06249 \& \&$ xo $1<0.0928564$

$\mathrm{b} 2=(-3.787 * \mathrm{xo} 1)+2.2916$

elseif xo $1>=0.0928564 \& \&$ xo $1<0.25$

Appendix A

$$
\text { b2 }=(-2.48 * x o 1)+2.17062 ; \text {. }
$$

elseif xo $1>=0.25 \& \&$ xo $1<0.5$

$$
\mathrm{b} 2=(-0.4 * \mathrm{xo} 1)+1.65
$$

elseif xo1 $=0$

$\mathrm{b} 2=0$;

end

$\mathrm{Eg} 1=\mathrm{xo} 1 * 1.9+(1-\mathrm{xo} 1) * 3.45-\mathrm{b} 2 * \mathrm{xo} 1 *(1-\mathrm{xo} 1) ; \quad$ Appendix A

$\mathrm{k}=1$;

for $\mathrm{xo}=0.09: 0.05: 0.5$

if $\mathrm{xo}>=0.01 \& \& \mathrm{xo}<0.06249$

$\mathrm{b} 1=(-10.64 * \mathrm{xo})+2.72377$;

elseif $\mathrm{xo}>=0.06249 \& \&$ xo $<0.0928564$ 


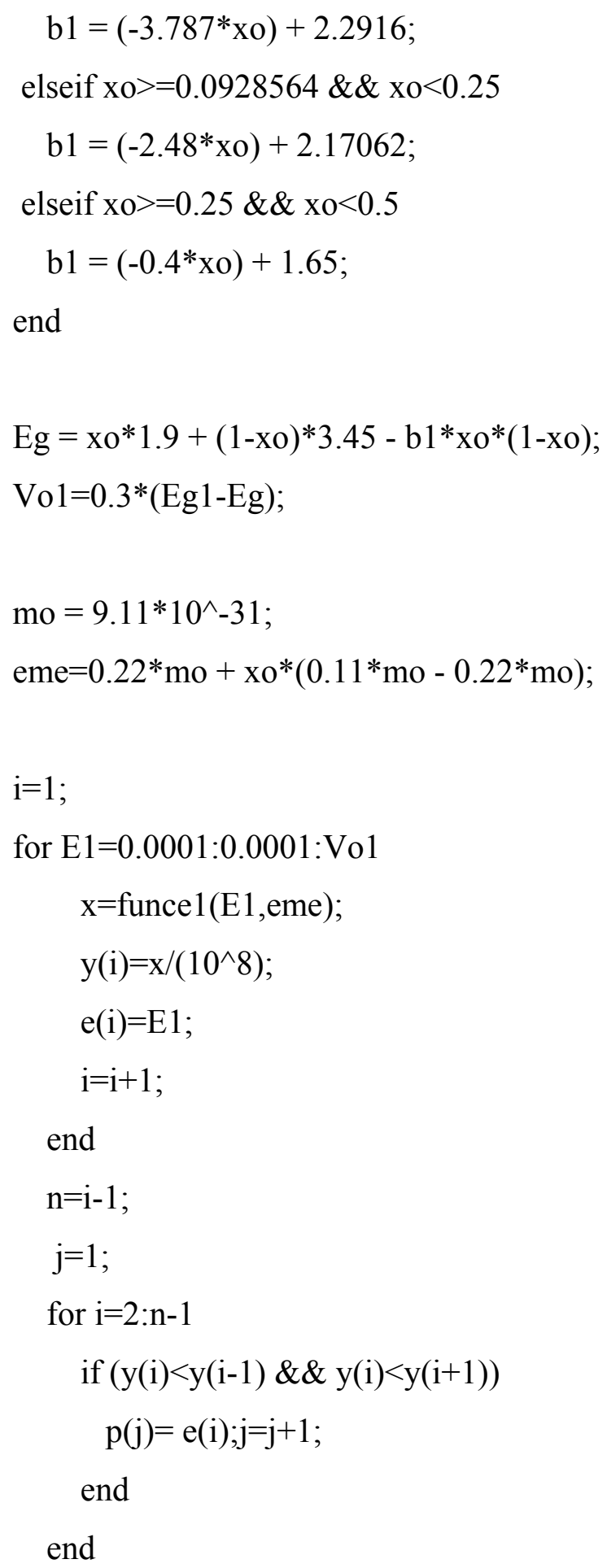

This is to compute the cot part of the solution 


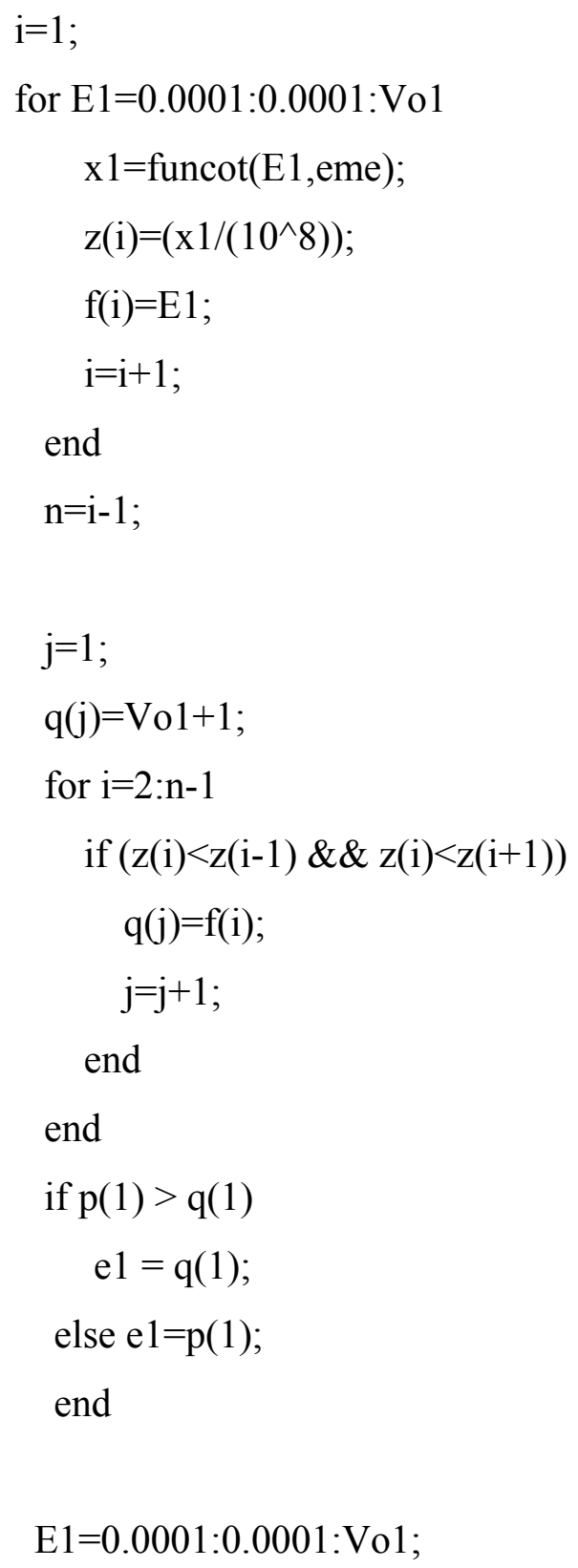

This is the calculation for the heavy hole

$$
\mathrm{eme}=\mathrm{mo} * 1.595+\mathrm{xo} *(1.449-1.595) * \mathrm{mo}
$$

Vo1=0.7*(Eg1-Eg);

This is to compute the tan part of the solution 


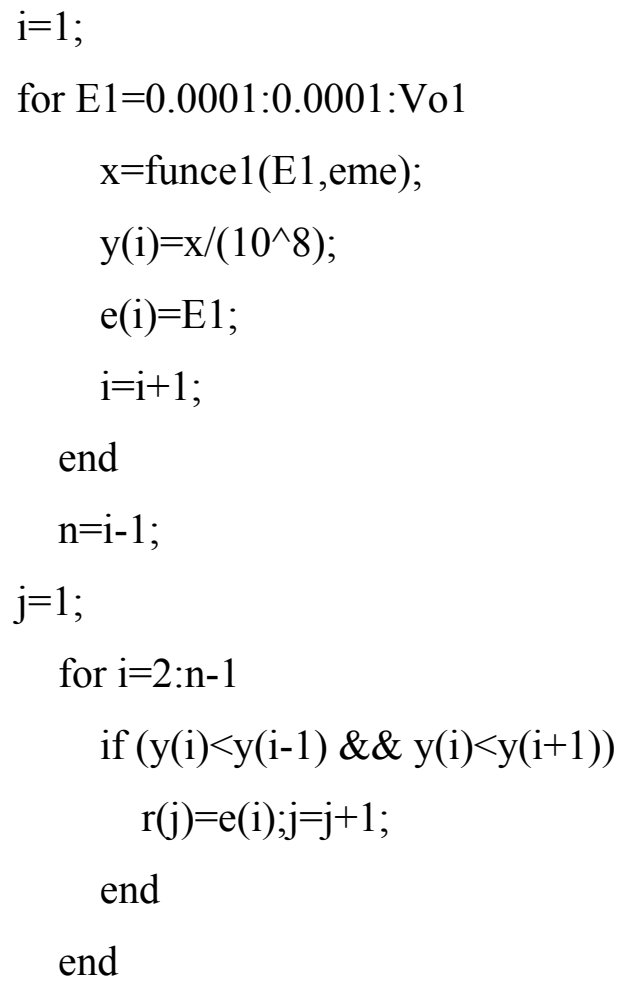




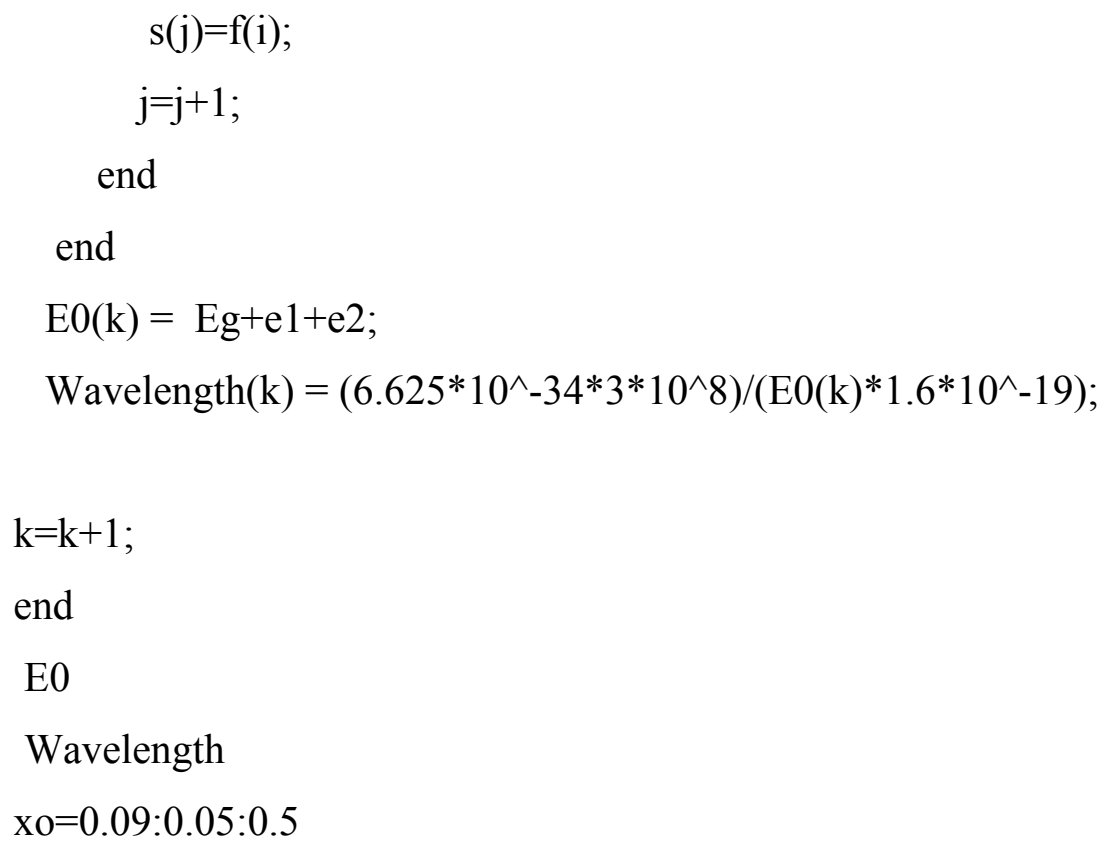




\section{APPENDIX D: PROTOCOL FOR DEPOSITING STRIPES OF}

\section{CHROME ON GaN}

\section{PHOTOLITHOGRAPHY FOR STRIPE PATTERN}

1. Clean the $\mathrm{GaN}$ sample (sample $=\mathrm{GaN}+$ sapphire , shiny surface is $\mathrm{GaN}$ and the dull surface is sapphire) grown on sapphire first by dipping the sample in acetone for $5 \mathrm{~min}$.

2. Remove the sample from acetone and dip the sample in methanol for $5 \mathrm{~min}$.

3. Remove the sample from methanol and dry it with $\mathrm{N}_{2}$ gas.

4. Place the dried sample in a petri dish with the $\mathrm{GaN}$ facing upwards and keep it in the oven for at least $30 \mathrm{~min}$ to remove any moisture present on the GaN surface.

5. In mean time switch on the MA6 mask aligner and wait for 30min.

6. Now turn on the monitor and conduct a lamp test to determine the power transmitted through the transparent part of the mask. Fro stripe pattern use the mask labeled laser mask. Point to be noted here is that the MA6 mask aligner is calibrated from time to time so one needs to make sure of the power transmitted through the mask before setting the exposure time.

7. Record the reading on the power meter during the lamp test. To calculate the time of exposure divide the energy (available from datasheets) required for exposing AZ5241 (positive photoresist). This will give you the time of exposure.

8. In the program set the time as this value.

9. Load the mask into the MA6 mask aligner.

10. Mix the developer-MIF 312 in ratio 1:1.2, 1 part developer and 1.2 parts de ionized water. An $80 \mathrm{ml}$ beaker should be sufficient as the size of the sample is small. Fill another $80 \mathrm{ml}$ beaker with de ionized water. St them aside in the solvent hood.

11. Remove the sample from the open allow it to cool down for $3 \mathrm{~min}$.

12. Spin AZ5241 photoresist at a speed of 5500rpm. This will give a photoresist thickness of $1.5 \mu \mathrm{m}$.

13. Load this sample into MA6 mask aligner and expose it.

14. Develop the exposed sample. 
15. Dip it in water to remove excess developer.

16. Dry the sample thoroughly to remove water.

\section{SPUTTERING CHROME ON THE PATTERENED SAMPLE}

1. Now load chrome target into the sputtering station and load the patterned sample as well.

2. Set the time for sputtering as $3.5 \mathrm{~min}$ at a power of $0.1 \mathrm{KW}$ (Refer to the log book to get the exact timings and powers. This should give a chrome thickness of $160 \mathrm{~nm}$.

3. Remove the sample from the machine and dip it in acetone for the lift off process. If chrome still sticks to the sample do an ultrasonic bath for a few seconds 2-3 seconds. Repeat the ultrasonic bath procedure if necessary.

4. The sample which you will be using is large enough to be cut into many pieces. Each piece may end up having 5 lines on it.

5. Now one single sample (uncut piece) when cut into many pieces can be used for performing multiple ICP-RIE processes. 